\title{
Redox Potentials of Group 13 Metal-substituted Dipnictenes: A comparative Cyclic Voltammetry Study
}

\author{
Hanns M. Weinert, ${ }^{\dagger}$ Christoph Wölper, ${ }^{\dagger}$ and Stephan Schulz ${ }^{* \dagger \#}$
}

IInstitute for Inorganic Chemistry and "Center for Nanointegration Duisburg-Essen (Cenide), University of Duisburg-Essen, 45117 Essen, Germany 


\section{Content}

Fig. S1-S3. ${ }^{1} \mathrm{H},{ }^{13} \mathrm{C}$ and ATR-IR spectrum of $\left[\mathrm{L}\left(\mathrm{Me}_{2} \mathrm{~N}\right) \mathrm{AlSb}\right]_{2} 8$.

Fig. S4-S12. ${ }^{1} \mathrm{H},{ }^{13} \mathrm{C}, \mathrm{COSY}, \mathrm{HSQC}, \mathrm{DOSY}$, ATR-IR and powder-XRD of [L(Et $\left.\left.{ }_{2} \mathrm{~N}\right) \mathrm{AlBi}\right]_{2} 9$.

Fig. S13-S15. ${ }^{1} \mathrm{H},{ }^{13} \mathrm{C}$ and ATR-IR spectrum of $\left[\mathrm{L}\left(\mathrm{Me}_{2} \mathrm{~N}\right) \mathrm{GaBi}\right]_{2} \mathbf{1 0}$.

Fig. S16-S18. ${ }^{1} \mathrm{H},{ }^{13} \mathrm{C}$ and ATR-IR spectrum of $[\mathrm{L}(\mathrm{EtO}) \mathrm{GaBi}]_{2} 11$.

Fig. S19-S21. ${ }^{1} \mathrm{H},{ }^{13} \mathrm{C}$ and ATR-IR spectrum of $\mathrm{LAl}\left(\mathrm{NMe}_{2}\right)_{2} 12$.

Fig. S22-S24. ${ }^{1} \mathrm{H},{ }^{13} \mathrm{C}$ and ATR-IR spectrum of $\mathrm{LAl}\left(\mathrm{NEt}_{2}\right)_{2} 13$.

Fig. S25-S27. ${ }^{1} \mathrm{H},{ }^{13} \mathrm{C}$ and ATR-IR spectrum of $\mathrm{LGa}\left(\mathrm{NEt}_{2}\right)_{2} 14$.

Fig. S28-S30. ${ }^{1} \mathrm{H},{ }^{13} \mathrm{C}$ and ATR-IR spectrum of $\left[\{\mathrm{L}(\mathrm{EtO}) \mathrm{Ga}\}_{2}-\mu, \eta^{1: 1}-\mathrm{Bi}_{4}\right] 15$.

Fig. S31. ${ }^{1} \mathrm{H}$ NMR spectra of the reaction of $\mathrm{LAl}$ and $\mathrm{Sb}\left(\mathrm{NMe}_{2}\right)_{3}$ at $-30{ }^{\circ} \mathrm{C}$ in $\mathrm{C}_{7} \mathrm{D}_{8}$.

Fig. S32. ${ }^{1} \mathrm{H}$ NMR spectra of the reaction of $\mathrm{LAl}$ and $\mathrm{Bi}\left(\mathrm{NEt}_{2}\right)_{3}$ at $-30{ }^{\circ} \mathrm{C}$ in $\mathrm{C}_{7} \mathrm{D}_{8}$.

Fig. S33. Temperature dependent ${ }^{1} \mathrm{H}$ NMR spectra $\left(500 \mathrm{MHz}, \mathrm{C}_{6} \mathrm{D}_{6}\right)$ of $\left[\mathrm{L}\left(\mathrm{Et}_{2} \mathrm{~N}\right) \mathrm{AlBi}\right]_{2} 9$.

Fig. S34. ${ }^{1} \mathrm{H}$ NMR spectrum $\left(300 \mathrm{MHz}, \mathrm{C}_{6} \mathrm{D}_{6}, 25^{\circ} \mathrm{C}\right)$ of the filtrate from $\left[\mathrm{L}\left(\mathrm{Me}_{2} \mathrm{~N}\right) \mathrm{GaBi}\right]_{2} \mathbf{1 0}$ synthesis.

Fig. S35. ${ }^{1} \mathrm{H}$ NMR spectra $\left(300 \mathrm{MHz}, \mathrm{C}_{6} \mathrm{D}_{6}, 25^{\circ} \mathrm{C}\right)$ of the reaction of $\mathrm{LGa}$ and $\mathrm{Bi}(\mathrm{OEt})_{3}$.

Fig. S36. ${ }^{1} \mathrm{H}$ NMR spectrum $\left(300 \mathrm{MHz}, \mathrm{C}_{7} \mathrm{D}_{8}, 25^{\circ} \mathrm{C}\right)$ of the reaction of $\left[\mathrm{L}\left(\mathrm{Me}_{2} \mathrm{~N}\right) \mathrm{AlSb}\right]_{2} 8$ and 2 eq. of $\mathrm{Fc}\left[\mathrm{B}\left(\mathrm{C}_{6} \mathrm{~F}_{5}\right)_{4}\right]$.

Fig. S37. Temperature dependent ${ }^{1} \mathrm{H}$ NMR spectra $\left(300 \mathrm{MHz}, \mathrm{C}_{7} \mathrm{D}_{8}\right)$ of $[\mathrm{L}(\mathrm{EtO}) \mathrm{GaBi}]_{2} 11$.

Fig. S38. ${ }^{1} \mathrm{H}$ NMR spectrum $\left(300 \mathrm{MHz}, \mathrm{C}_{7} \mathrm{D}_{8}, 25^{\circ} \mathrm{C}\right)$ after thermal treatment $(24 \mathrm{~h})$ of a solution of $[\mathrm{L}(\mathrm{EtO}) \mathrm{GaBi}]_{2} \mathbf{1 1}$ at $100{ }^{\circ} \mathrm{C}$.

Fig. S39. ${ }^{1} \mathrm{H}$ NMR spectra $\left(300 \mathrm{MHz}, \mathrm{C}_{7} \mathrm{D}_{8}, 25{ }^{\circ} \mathrm{C}\right)$ of $\left[\mathrm{L}\left(\mathrm{Me}_{2} \mathrm{~N}\right) \mathrm{AlSb}\right]_{2} 8$ after heating to $100{ }^{\circ} \mathrm{C}$ for $1 \mathrm{~min}, 30 \mathrm{~min}$ and $24 \mathrm{~h}$ in a aluminum heating block.

Fig. S40. ${ }^{1} \mathrm{H}$ NMR spectrum $\left(300 \mathrm{MHz}, \mathrm{C}_{7} \mathrm{D}_{8}, 25^{\circ} \mathrm{C}\right)$ after thermal treatment $(24 \mathrm{~h})$ of a solution of $\left[\mathrm{L}\left(\mathrm{Me}_{2} \mathrm{~N}\right) \mathrm{GaBi}\right]_{2} \mathbf{1 0}$ at $100{ }^{\circ} \mathrm{C}$.

Fig. S41-S51. Cyclic voltammograms of 1-11 in THF solution.

Fig. S52. Cyclic voltammograms of $[\mathrm{L}(\mathrm{TfO}) \mathrm{GaBi}]_{2}$ in THF solution.

Fig. S53-S63. UV-VIS spectra of 1-11 in benzene solution.

Fig. S64 UV-VIS spectra of $[\mathrm{L}(\mathrm{Cl}) \mathrm{GaAs}]_{2} \mathbf{3}$ and $\mathrm{LGa}(\mathrm{Cl})_{2}$.

Fig. S65. Molecular structure of LAl( $\left.\mathrm{NEt}_{2}\right)_{2}$ in the crystal.

Fig. S66. ELF distribution in the $\left[\left(\mathrm{L}\left(\mathrm{Me}_{2} \mathrm{~N}\right) \mathrm{AlSb}\right]_{2} \mathbf{8},\left[\left(\mathrm{L}\left(\mathrm{Me}_{2} \mathrm{~N}\right) \mathrm{GaSb}\right]_{2} 4\right.\right.$ in the $\mathrm{M}-\mathrm{E}-\mathrm{E}-\mathrm{M}$ plane.

Table S1. ${ }^{1} \mathrm{H}$ NMR chemical shifts of $8-14$ in $\mathrm{C}_{6} \mathrm{D}_{6}$ of selected resonance.

Table S2. DOSY NMR results of $\left[\mathrm{L}\left(\mathrm{Et}_{2} \mathrm{~N}\right) \mathrm{AlBi}\right]_{2} \mathbf{9}$.

Table S3a \& S3b. Crystallographic data of $\left[\mathrm{L}\left(\mathrm{Me}_{2} \mathrm{~N}\right) \mathrm{AlSb}\right]_{2}(\mathbf{8}),\left[\mathrm{L}\left(\mathrm{Et}_{2} \mathrm{~N}\right) \mathrm{AlBi}\right]_{2}(\mathbf{9})$, $\left[\mathrm{L}\left(\mathrm{Me}_{2} \mathrm{~N}\right) \mathrm{GaBi}\right]_{2}(\mathbf{1 0}),[\mathrm{L}(\mathrm{EtO}) \mathrm{GaBi}]_{2}(\mathbf{1 1}), \mathrm{LAl}\left(\mathrm{NEt}_{2}\right)_{2}(\mathbf{1 3}),\left[\left\{\mathrm{L}(\mathrm{EtO}) \mathrm{Ga}_{2}-\mu, \eta^{1: 1}-\mathrm{Bi}_{4}\right]\right.$ (15).

Table S4-7. Calculated X-Y bond lengths (r, $\AA$ ), NBO- and AIM-analysis of [(L( $\left.\left(\mathrm{Me}_{2} \mathrm{~N}\right) \mathrm{AlSb}\right]_{2} \mathbf{8}$, $\left[\left(\mathrm{L}\left(\mathrm{Me}_{2} \mathrm{~N}\right) \mathrm{GaSb}\right]_{2} 4\left[\left(\mathrm{~L}\left(\mathrm{Et}_{2} \mathrm{~N}\right) \mathrm{AlBi}\right]_{2} 9\right.\right.$ and $\left[\left(\mathrm{L}_{\left.\left(\mathrm{Et}_{2} \mathrm{~N}\right) \mathrm{GaBi}\right]_{2}} 7\right.\right.$. 


\section{Experimental Details}

\section{Isolation and spectroscopic characterization of by-products $L M\left(N_{2}\right)_{2}$ 12-14. $^{2}$}

$\left[\mathrm{L}\left(\mathrm{Et}_{2} \mathrm{~N}\right) \mathrm{GaBi}\right]_{2}(\mathbf{7}),\left[\mathrm{L}\left(\mathrm{Me}_{2} \mathrm{~N}\right) \mathrm{AlSb}\right]_{2}(\mathbf{8})$, and $\left[\mathrm{L}\left(\mathrm{Et}_{2} \mathrm{~N}\right) \mathrm{AlBi}\right]_{2}(\mathbf{9})$ were synthesized and isolated as described. The remaining filtrates were evaporated to dryness and the resulting residues dissolved in minimal amounts of $n$-hexane and stored at $-30{ }^{\circ} \mathrm{C}$. $\mathrm{LM}\left(\mathrm{NR}_{2}\right)_{2} \mathbf{1 2}-14$ precipitated as yellowish solids, which were recrystallized once more from $n$-hexane and isolated by filtration.

LAl(NMe2)2 (12). Yellowish crystalline solid; yield: $24 \mathrm{mg}(47 \mu \mathrm{mol}, 46 \%)$. Anal. Calcd. for $\mathrm{C}_{33} \mathrm{H}_{53} \mathrm{AlN}_{4}$ : C, 74.39; H, 10.03; N, 10.52. Found: C, 74.2; H, 9.82 N, 10.3. \%. ATR-IR: v 2967, 2869, 2822, 1551, 1516, 1438, 1391, 1316, 1256, 1174, 1103, 1013, 797, 758, 601, 451, $390 \mathrm{~cm}^{-1}$. ${ }^{1} \mathbf{H}$ NMR $\left.\left(400 \mathrm{MHz}, \mathrm{C}_{6} \mathrm{D}_{6}, 25^{\circ} \mathrm{C}\right): \delta 7.19-7.10\left(\mathrm{~m}, 12 \mathrm{H}, \mathrm{C}_{6} H_{3}\right)-2,6^{i} \operatorname{Pr}_{2}\right), 4.89(\mathrm{~s}, 2 \mathrm{H}, \gamma-\mathrm{CH}), 3.51$ (sept, $\left.{ }^{3} J_{\mathrm{HH}}=6.8 \mathrm{~Hz}, 4 \mathrm{H}, \mathrm{CH}\left(\mathrm{CH}_{3}\right)_{2}\right), 3.35\left(\mathrm{sept},{ }^{3} J_{\mathrm{HH}}=6.9 \mathrm{~Hz}, 4 \mathrm{H}, \mathrm{CH}\left(\mathrm{CH}_{3}\right)_{2}\right), 2.70(\mathrm{~s}, 6 \mathrm{H}$, $\left.\left.\mathrm{N}\left(\mathrm{CH}_{3}\right)_{2}\right), 2.71\left(\mathrm{~s}, 6 \mathrm{H}, \mathrm{N}\left(\mathrm{CH}_{3}\right)_{2}\right), 2.14\left(\mathrm{~s}, 12 \mathrm{H}, \mathrm{N}\left(\mathrm{CH}_{3}\right)_{2}\right), 1.54(\mathrm{~s}, 12 \mathrm{H}, \operatorname{ArNCCH})_{3}\right), 1.41\left(\mathrm{~d},{ }^{3} J_{\mathrm{HH}}\right.$ $\left.=6.7 \mathrm{~Hz}, 12 \mathrm{H}, \mathrm{CH}\left(\mathrm{CH}_{3}\right)_{2}\right), 1.35\left(\mathrm{~d},{ }^{3} J_{\mathrm{HH}}=6.8 \mathrm{~Hz}, 12 \mathrm{H}, \mathrm{CH}\left(\mathrm{CH}_{3}\right)_{2}\right), 1.27\left(\mathrm{~d},{ }^{3} J_{\mathrm{HH}}=6.9 \mathrm{~Hz}, 12 \mathrm{H}\right.$, $\left.\mathrm{CH}\left(\mathrm{CH}_{3}\right)_{2}\right), 1.10\left(\mathrm{~d},{ }^{3} J_{\mathrm{HH}}=6.9 \mathrm{~Hz}, 12 \mathrm{H}, \mathrm{CH}\left(\mathrm{CH}_{3}\right)_{2}\right) .{ }^{13} \mathbf{C ~ N M R}\left(100.7 \mathrm{MHz}, \mathrm{C}_{6} \mathrm{D}_{6}, 25{ }^{\circ} \mathrm{C}\right): \delta 169.5$ $\left(\mathrm{ArNCCH}_{3}\right), 145.0,144.1,142.3,127.3,124.8,124.2(\mathrm{ArC}), 99.0(\gamma-\mathrm{CH}), 42.16,42.0,41.2$ $\left(\mathrm{N}\left(\mathrm{CH}_{3}\right)_{2}\right), 29.0,28.1\left(\mathrm{CH}\left(\mathrm{CH}_{3}\right)_{2}\right), 25.7,25.0,24.8,24.4\left(\mathrm{CH}\left(\mathrm{CH}_{3}\right)_{2}\right) 23.5\left(\mathrm{ArNCCH}_{3}\right)$.

LAl(NEt $)_{2}$ 2 (13). Yellowish crystalline solid; yield: $50 \mathrm{mg}(31 \mu \mathrm{mol}, 20 \%)$. Anal. Calcd. for $\mathrm{C}_{37} \mathrm{H}_{61} \mathrm{AlN}_{4}$ : C, 75.46; H, 10.44; N, 9.47. Found: C, 75.40; H, 10.7; N, 9.51 \%. ATR-IR: v 3061, 2959, 2865, 2821, 1619, 1517, 1383, 1369, 1314, 1254, 1161, 1015, 797, 757, 654, 607, 483, 436 $\mathrm{cm}^{-1} .{ }^{1} \mathbf{H}$ NMR $\left(400 \mathrm{MHz}, \mathrm{C}_{6} \mathrm{D}_{6}, 25{ }^{\circ} \mathrm{C}\right): \delta 7.21\left(\mathrm{dd},{ }^{4} J_{\mathrm{HH}}=7.7 \mathrm{~Hz},{ }^{5} J_{\mathrm{HH}}=2.2 \mathrm{~Hz}, 2 \mathrm{H}, \mathrm{C}_{6} H_{3}\right)-$ $\left.\left.2,6^{i} \operatorname{Pr}_{2}\right), 7.18\left(\mathrm{t},{ }^{4} J_{\mathrm{HH}}=7.4 \mathrm{~Hz}, 2 \mathrm{H}, \mathrm{C}_{6} H_{3}\right)-2,6^{i} \operatorname{Pr}_{2}\right), 6.99\left(\mathrm{dd},{ }^{4} J_{\mathrm{HH}}=7.2 \mathrm{~Hz},{ }^{5} J_{\mathrm{HH}}=2.1 \mathrm{~Hz}, 2 \mathrm{H}\right.$, $\left.\left.\mathrm{C}_{6} H_{3}\right)-2,6{ }^{i} \mathrm{Pr}_{2}\right), 4.93(\mathrm{~s}, 1 \mathrm{H}, \gamma-\mathrm{CH}), 4.00\left(\mathrm{sept},{ }^{3} J_{\mathrm{HH}}=6.8 \mathrm{~Hz}, 2 \mathrm{H}, \mathrm{CH}\left(\mathrm{CH}_{3}\right)_{2}\right), 3.23(\mathrm{sept}+\mathrm{q}, 4 \mathrm{H}$, $\mathrm{CH}\left(\mathrm{CH}_{3}\right)_{2}$ and $\left.\mathrm{N}\left(\mathrm{CH}_{2} \mathrm{CH}_{3}\right)_{2}\right), 3.11\left(\mathrm{q},{ }^{3} J_{\mathrm{HH}}=6.9 \mathrm{~Hz}, 2 \mathrm{H}, \mathrm{N}\left(\mathrm{CH}_{2} \mathrm{CH}_{3}\right)_{2}\right), 2.76\left(\mathrm{q},{ }^{3} J_{\mathrm{HH}}=6.9 \mathrm{~Hz}, 4\right.$ $\left.\mathrm{H}, \mathrm{N}\left(\mathrm{CH}_{2} \mathrm{CH}_{3}\right)_{2}\right), 1.54(\mathrm{~s}, 6 \mathrm{H}, \mathrm{ArNCCH})_{3}, 1.48\left(\mathrm{~d},{ }^{3} \mathrm{~J}_{\mathrm{HH}}=6.6 \mathrm{~Hz}, 6 \mathrm{H}, \mathrm{CH}\left(\mathrm{CH}_{3}\right)_{2}\right), 1.33\left(\mathrm{~d},{ }^{3} J_{\mathrm{HH}}=\right.$ $\left.6.8 \mathrm{~Hz}, 6 \mathrm{H}, \mathrm{CH}\left(\mathrm{CH}_{3}\right)_{2}\right), 1.25\left(\mathrm{~d},{ }^{3} J_{\mathrm{HH}}=6.8 \mathrm{~Hz}, 6 \mathrm{H}, \mathrm{CH}\left(\mathrm{CH}_{3}\right)_{2}\right), 1.23\left(\mathrm{t},{ }^{3} J_{\mathrm{HH}}=7.0 \mathrm{~Hz}, 3 \mathrm{H}\right.$, $\left.\mathrm{N}\left(\mathrm{CH}_{2} \mathrm{CH}_{3}\right)_{2}\right), 1.15\left(\mathrm{t},{ }^{3} J_{\mathrm{HH}}=6.9 \mathrm{~Hz}, 3 \mathrm{H}, \mathrm{N}\left(\mathrm{CH}_{2} \mathrm{CH}_{3}\right)_{2}\right), 1.10\left(\mathrm{~d},{ }^{3} J_{\mathrm{HH}}=6.8 \mathrm{~Hz}, 6 \mathrm{H}, \mathrm{CH}\left(\mathrm{CH}_{3}\right)_{2}\right)$, $0.47\left(\mathrm{t},{ }^{3} J_{\mathrm{HH}}=6.9 \mathrm{~Hz}, 6 \mathrm{H}, \mathrm{N}\left(\mathrm{CH}_{2} \mathrm{CH}_{3}\right)_{2}\right) .{ }^{13} \mathbf{C} \mathbf{N M R}\left(100.7 \mathrm{MHz}, \mathrm{C}_{6} \mathrm{D}_{6}, 25{ }^{\circ} \mathrm{C}\right): \delta 170.3$, $\left(\mathrm{ArNCCH}_{3}\right), 145.3,143.9,143.1,127.1,125.0,124.4(\mathrm{ArC}), 99.1(\gamma-\mathrm{CH}), 41.0,40.7,40.1$, $\left(\mathrm{N}\left(\mathrm{CH}_{2} \mathrm{CH}_{3}\right)_{2}\right), 28.9,27.1\left(\mathrm{CH}\left(\mathrm{CH}_{3}\right)_{2}\right), 26.1,25.7,25.1,24.9\left(\mathrm{CH}\left(\mathrm{CH}_{3}\right)_{2}\right), 24.2,(\mathrm{ArNCCH} 3), 14.3$, 14.2, 13.6. $\left(\mathrm{N}\left(\mathrm{CH}_{2} \mathrm{CH}_{3}\right)_{2}\right)$.

LGa(NEt2)2 (14). Colorless crystalline solid; yield: $201 \mathrm{mg}(31 \mu \mathrm{mol}, 22 \%)$. Anal. Calcd. for $\mathrm{C}_{37} \mathrm{H}_{61} \mathrm{GaN}_{4}$ : C, 70.36; H, 9.73; N, 8.87. Found: C, 70.20; H, 9.99; N, 8.84 \%. ATR-IR: v 3061, 2962, 2924, 2868, 2818, 1551, 1517, 1438, 1393, 1372, 1316, 1258, 1173, 1013, 931, 796, 757, 585, $467 \mathrm{~cm}^{-1} .{ }^{1} \mathbf{H}$ NMR $\left(400 \mathrm{MHz}, \mathrm{C}_{6} \mathrm{D}_{6}, 25^{\circ} \mathrm{C}\right): \delta 7.18\left(\mathrm{dd},{ }^{4} J_{\mathrm{HH}}=7.6 \mathrm{~Hz},{ }^{5} J_{\mathrm{HH}}=2.3 \mathrm{~Hz}, 2 \mathrm{H}, \mathrm{C}_{6} H_{3}\right)-$ 2, $\left.\left.{ }^{i} \operatorname{Pr}_{2}\right), 7.15\left(\mathrm{t},{ }^{4} J_{\mathrm{HH}}=7.5 \mathrm{~Hz}, 2 \mathrm{H}, \mathrm{C}_{6} H_{3}\right)-2,6^{i} \operatorname{Pr}_{2}\right), 7.11\left(\mathrm{dd},{ }^{4} J_{\mathrm{HH}}=7.2 \mathrm{~Hz},{ }^{5} J_{\mathrm{HH}}=2.4 \mathrm{~Hz}, 2 \mathrm{H}\right.$, $\left.\left.\mathrm{C}_{6} \mathrm{H}_{3}\right)-2,6^{i} \mathrm{Pr}_{2}\right), 4.75(\mathrm{~s}, 1 \mathrm{H}, \gamma-\mathrm{CH}), 3.94\left(\mathrm{sept},{ }^{3} \mathrm{~J}_{\mathrm{HH}}=6.7 \mathrm{~Hz}, 2 \mathrm{H}, \mathrm{CH}\left(\mathrm{CH}_{3}\right)_{2}\right), 3.31\left(\mathrm{sept},{ }^{3} J_{\mathrm{HH}}=6.6\right.$ $\left.\mathrm{Hz}, 2 \mathrm{H}, \mathrm{CH}\left(\mathrm{CH}_{3}\right)_{2}\right), 3.29$ (overlayed, $\left.2 \mathrm{H}, \mathrm{N}\left(\mathrm{CH}_{2} \mathrm{CH}_{3}\right)_{2}\right), 3.25\left(\mathrm{q},{ }^{3} J_{\mathrm{HH}}=6.7 \mathrm{~Hz}, 2 \mathrm{H}, \mathrm{N}\left(\mathrm{CH}_{2} \mathrm{CH}_{3}\right)_{2}\right)$, $2.75\left(\mathrm{q},{ }^{3} \mathrm{~J}_{\mathrm{HH}}=6.9 \mathrm{~Hz}, 4 \mathrm{H}, \mathrm{N}\left(\mathrm{CH}_{2} \mathrm{CH}_{3}\right)_{2}\right), 1.53(\mathrm{~s}, 6 \mathrm{H}, \operatorname{ArNCCH}), 1.47\left(\mathrm{~d},{ }^{3} \mathrm{~J}_{\mathrm{HH}}=6.7 \mathrm{~Hz}, 6 \mathrm{H}\right.$, $\left.\mathrm{CH}\left(\mathrm{CH}_{3}\right)_{2}\right), 1.34\left(\mathrm{~d},{ }^{3} J_{\mathrm{HH}}=6.9 \mathrm{~Hz}, 6 \mathrm{H}, \mathrm{CH}\left(\mathrm{CH}_{3}\right)_{2}\right), 1.26\left(\mathrm{~d},{ }^{3} J_{\mathrm{HH}}=6.8 \mathrm{~Hz}, 6 \mathrm{H}, \mathrm{CH}\left(\mathrm{CH}_{3}\right)_{2}\right), 1.23(\mathrm{t}$, $\left.{ }^{3} J_{\mathrm{HH}}=6.7 \mathrm{~Hz}, 3 \mathrm{H}, \mathrm{N}\left(\mathrm{CH}_{2} \mathrm{CH}_{3}\right)_{2}\right), 1.22\left(\mathrm{t},{ }^{3} J_{\mathrm{HH}}=6.8 \mathrm{~Hz}, 3 \mathrm{H}, \mathrm{N}\left(\mathrm{CH}_{2} \mathrm{CH}_{3}\right)_{2}\right), 1.13\left(\mathrm{~d},{ }^{3} J_{\mathrm{HH}}=6.8 \mathrm{~Hz}\right.$, $\left.6 \mathrm{H}, \mathrm{CH}\left(\mathrm{CH}_{3}\right)_{2}\right), 0.48\left(\mathrm{t},{ }^{3} \mathrm{~J}_{\mathrm{HH}}=6.9 \mathrm{~Hz}, 6 \mathrm{H}, \mathrm{N}\left(\mathrm{CH}_{2} \mathrm{CH}_{3}\right)_{2}\right) .{ }^{13} \mathbf{C} \mathbf{N M R}\left(100.7 \mathrm{MHz}, \mathrm{C}_{6} \mathrm{D}_{6}, 25{ }^{\circ} \mathrm{C}\right): \delta$ 170.1, $\left(\mathrm{ArNCCH}_{3}\right), 145.1,143.8$ 143.4, 127.0, 124.9, 124.3, 145.3, 143.9, 143.1, 127.1, 125.0, 124.4 $(\mathrm{ArC}), 97.8(\gamma-\mathrm{CH}), 43.5,43.0,42.9,\left(\mathrm{~N}\left(\mathrm{CH}_{2} \mathrm{CH}_{3}\right)_{2}\right), 28.8,27.3\left(\mathrm{CH}\left(\mathrm{CH}_{3}\right)_{2}\right), 25.9,25.6,25.0,24.9$

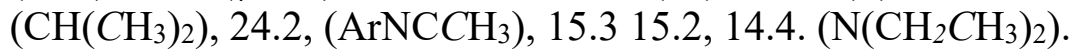


Table S1. ${ }^{1} \mathrm{H}$ NMR chemical shifts of $\mathbf{8}-\mathbf{1 4}$ in $\mathrm{C}_{6} \mathrm{D}_{6}$ of selected resonance.

\begin{tabular}{|c|c|c|c|c|c|}
\hline & $\gamma-\mathrm{CH}$ & $\mathrm{CHMe}$ & $\mathrm{NCCH}_{3}$ & $\mathrm{CHMe}$ & $\mathrm{LGa}\left[\mathrm{X}\left(\left[\mathrm{CH}_{2}\right] \mathrm{CH}_{3}\right)_{\mathrm{x}}\right]_{\mathrm{x}}$ \\
\hline$\left[\mathrm{L}\left(\mathrm{Me}_{2} \mathrm{~N}\right) \mathrm{AlSb}\right]_{2} \mathbf{8}$ & 4.96 & $3.60,2.93$ & 1.62 & $1.41,1.30,1.09,1.00$ & $2.85,2.69$ \\
\hline$\left[\mathrm{L}\left(\mathrm{Et}_{2} \mathrm{~N}\right) \mathrm{AlBi}\right]_{2} 9$ & $\begin{array}{l}4.96 \\
5.84 \\
5.04\end{array}$ & $\begin{array}{l}4.12,2.57 \\
4.29,3.47 \\
2.95,2.77\end{array}$ & $\begin{array}{l}1.58 \\
1.84 \\
1.60\end{array}$ & $\begin{array}{l}1.41,1.28,1.03,1.00 \\
1.59,1.39,1.341 .32 \\
1.29,1.15,1.13,1.10\end{array}$ & $\begin{array}{c}3.43,2.851 .29 .1 .03 \\
3.59,3.52,3.43 \\
1.56,1.39,0.37\end{array}$ \\
\hline$\left[\mathrm{L}\left(\mathrm{Me}_{2} \mathrm{~N}\right) \mathrm{GaBi}\right]_{2} \mathbf{1 0}$ & 4.75 & $3.64,2.82$ & 1.63 & $1.39,1.31,1.13,1.02$ & $3.47,2.60$ \\
\hline$[\mathrm{L}(\mathrm{EtO}) \mathrm{GaBi}]_{2} \mathbf{1 1}$ & 4.80 & $4.01,2.85$ & 1.63 & $1.38,1.39,1.08,1.04$ & $3.801,42$ \\
\hline $\mathrm{LAl}\left(\mathrm{NMe}_{2}\right)_{2} \mathbf{1 2}$ & 4.89 & $3.52,3.35$ & 1.54 & $1.41,1.35,1.27,1.10$ & $2.71,2.70,2.14$ \\
\hline $\mathrm{LAl}\left(\mathrm{NEt}_{2}\right)_{2} \mathbf{1 3}$ & 4.93 & $4.00,3.23$ & 1.54 & $1.48,1.33,1.25,1.11$ & $\begin{array}{c}3.22,3.11,2.76 \\
1.23,1.15,0.47\end{array}$ \\
\hline $\mathrm{LGa}\left(\mathrm{NEt}_{2}\right)_{2} 14$ & 4.75 & $3.94,3.30$ & 1.53 & $1.47,1.34,1.26,1.13$ & $\begin{array}{c}3.29,3.25,2.75 \\
1,23,1.22,0.48\end{array}$ \\
\hline$\left[\{\mathrm{L}(\mathrm{EtO}) \mathrm{Ga}\}_{2-} \mu, \eta^{1: 1}-\mathrm{Bi}_{4}\right] \mathbf{1 5}$ & 4.69 & $3.68,3.02$ & 1.55 & $1.50,1.38,1.29,1.04$ & $3.93,1.49$ \\
\hline
\end{tabular}



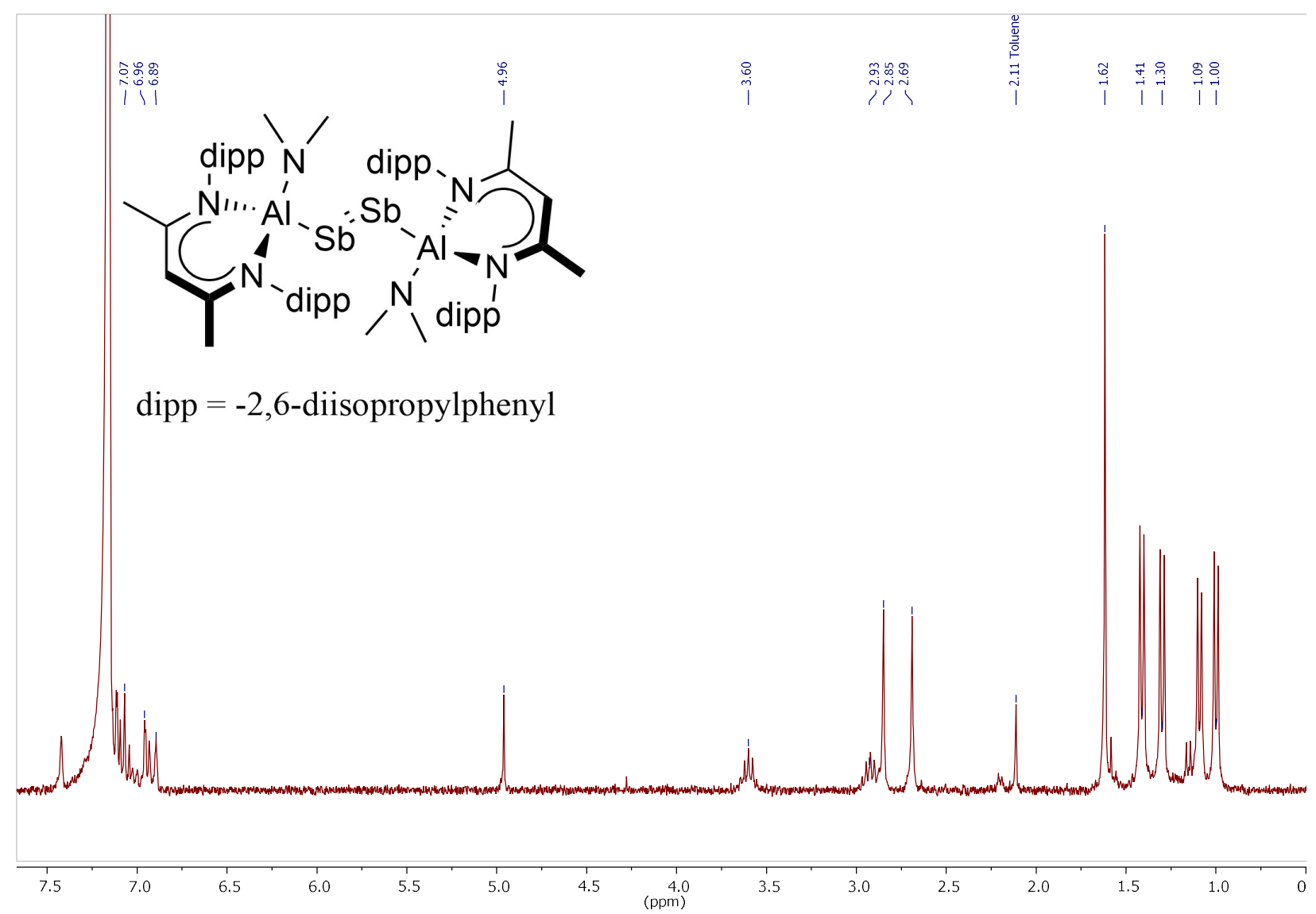

Fig. S1. ${ }^{1} \mathrm{H}$ NMR spectrum $\left(300 \mathrm{MHz}, \mathrm{C}_{6} \mathrm{D}_{6}, 25^{\circ} \mathrm{C}\right)$ of $\left[\mathrm{L}\left(\mathrm{Me}_{2} \mathrm{~N}\right) \mathrm{AlSb}\right]_{2} 8$.

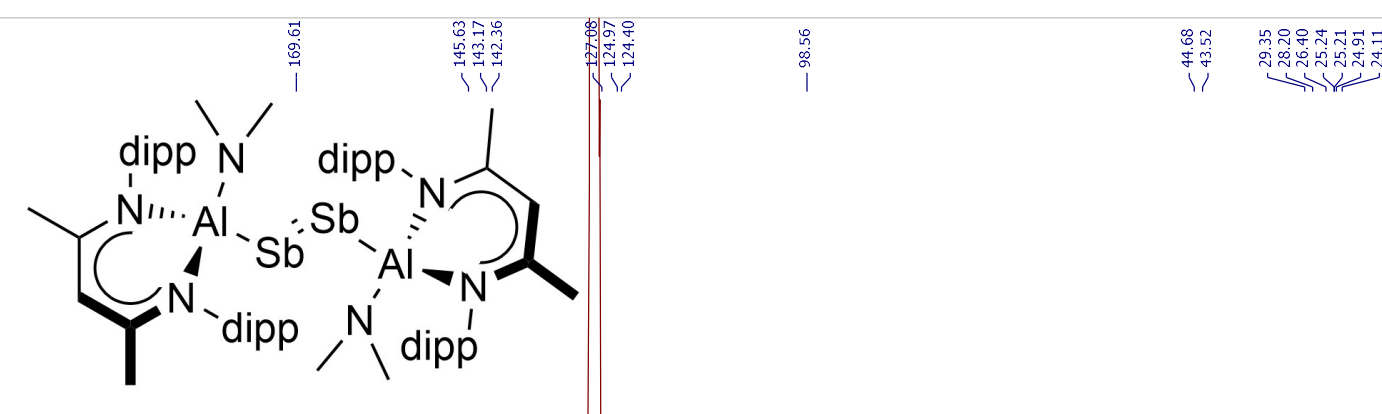

$\operatorname{dipp}=-2,6$-diisopropylphenyl

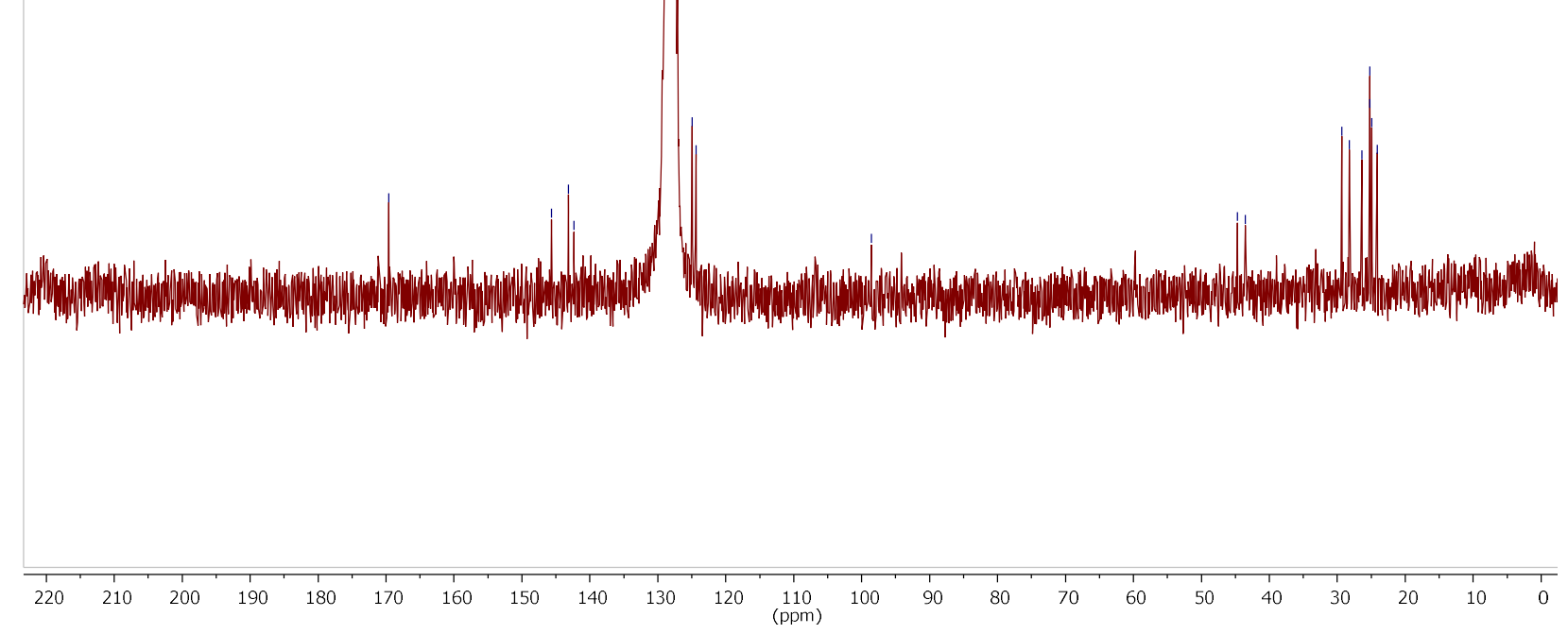

Fig. S2. ${ }^{13} \mathrm{C}$ NMR spectrum $\left(100.7 \mathrm{MHz}, \mathrm{C}_{6} \mathrm{D}_{6}, 25^{\circ} \mathrm{C}\right)$ of $\left[\mathrm{L}\left(\mathrm{Me}_{2} \mathrm{~N}\right) \mathrm{AlSb}\right]_{2} 8$. 


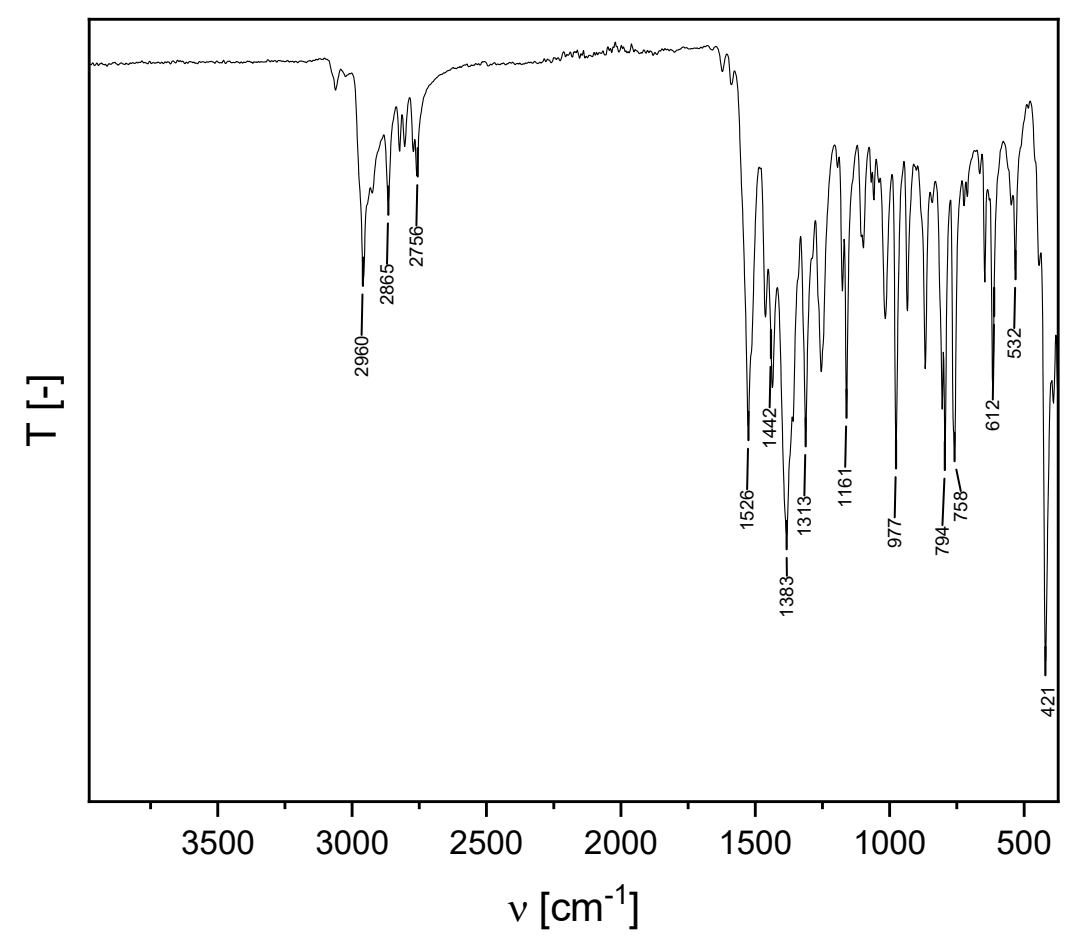

Fig. S3. ATR-IR spectrum of $\left[\mathrm{L}\left(\mathrm{Me}_{2} \mathrm{~N}\right) \mathrm{AlSb}\right]_{2} \mathbf{8}$.

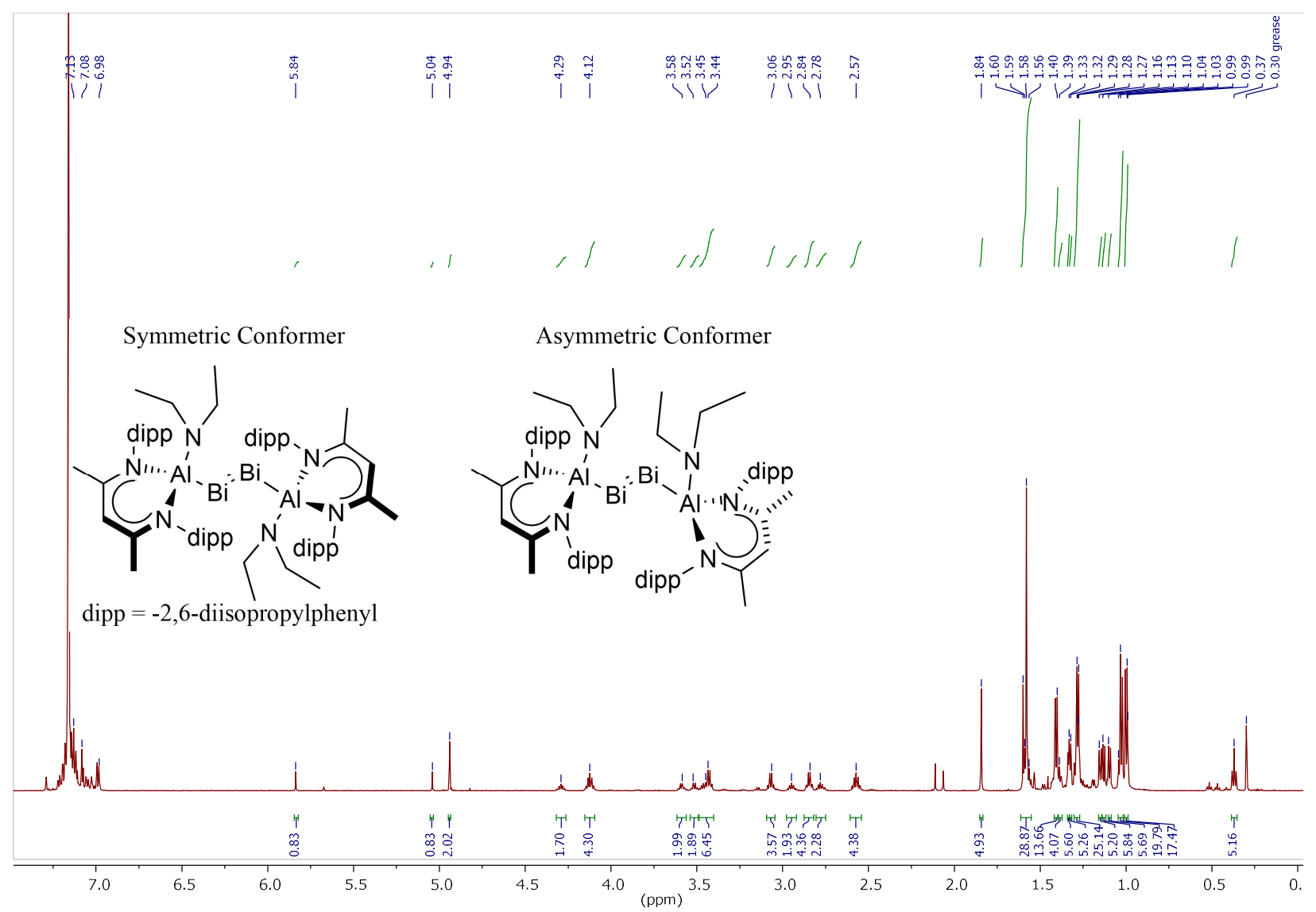

Fig. S4. ${ }^{1} \mathrm{H}$ NMR spectrum $\left(600 \mathrm{MHz}, \mathrm{C}_{6} \mathrm{D}_{6}, 25^{\circ} \mathrm{C}\right)$ of $\left[\mathrm{L}\left(\mathrm{Et}_{2} \mathrm{~N}\right) \mathrm{AlBi}\right]_{2} 9$. 


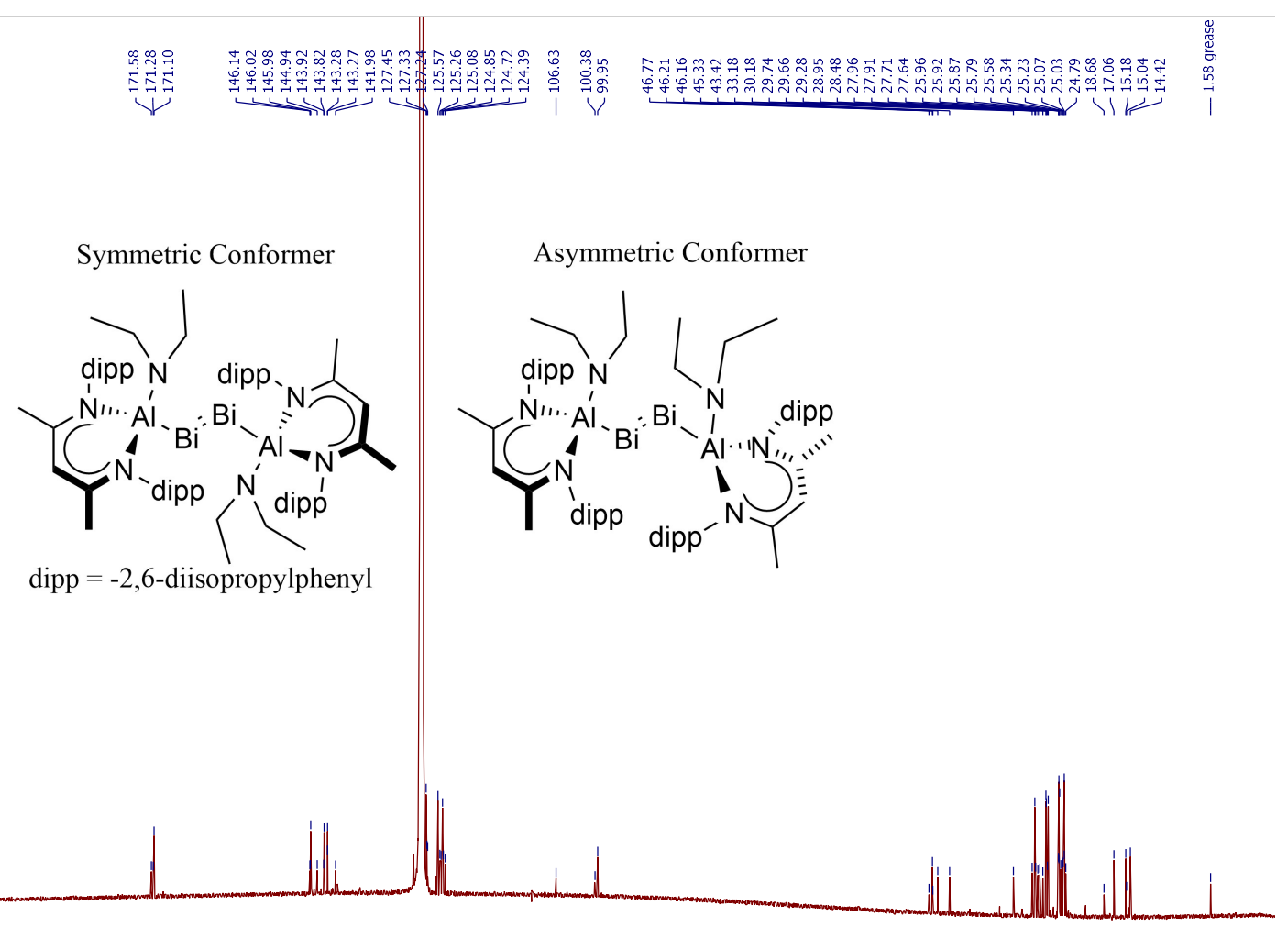

$\begin{array}{llllllllllllllllllllllllllll}220 & 210 & 200 & 190 & 180 & 170 & 160 & 150 & 140 & 130 & 120 & \begin{array}{c}110 \\ (\mathrm{ppm})\end{array} & 100 & 90 & 80 & 70 & 60 & 50 & 40 & 30 & 20 & 10 & 0\end{array}$

Fig. S5. ${ }^{13} \mathrm{C}$ NMR spectrum $\left(150.9 \mathrm{MHz}, \mathrm{C}_{6} \mathrm{D}_{6}, 25^{\circ} \mathrm{C}\right)$ of $\left[\mathrm{L}\left(\mathrm{Et}_{2} \mathrm{~N}\right) \mathrm{AlBi}\right]_{2} 9$.

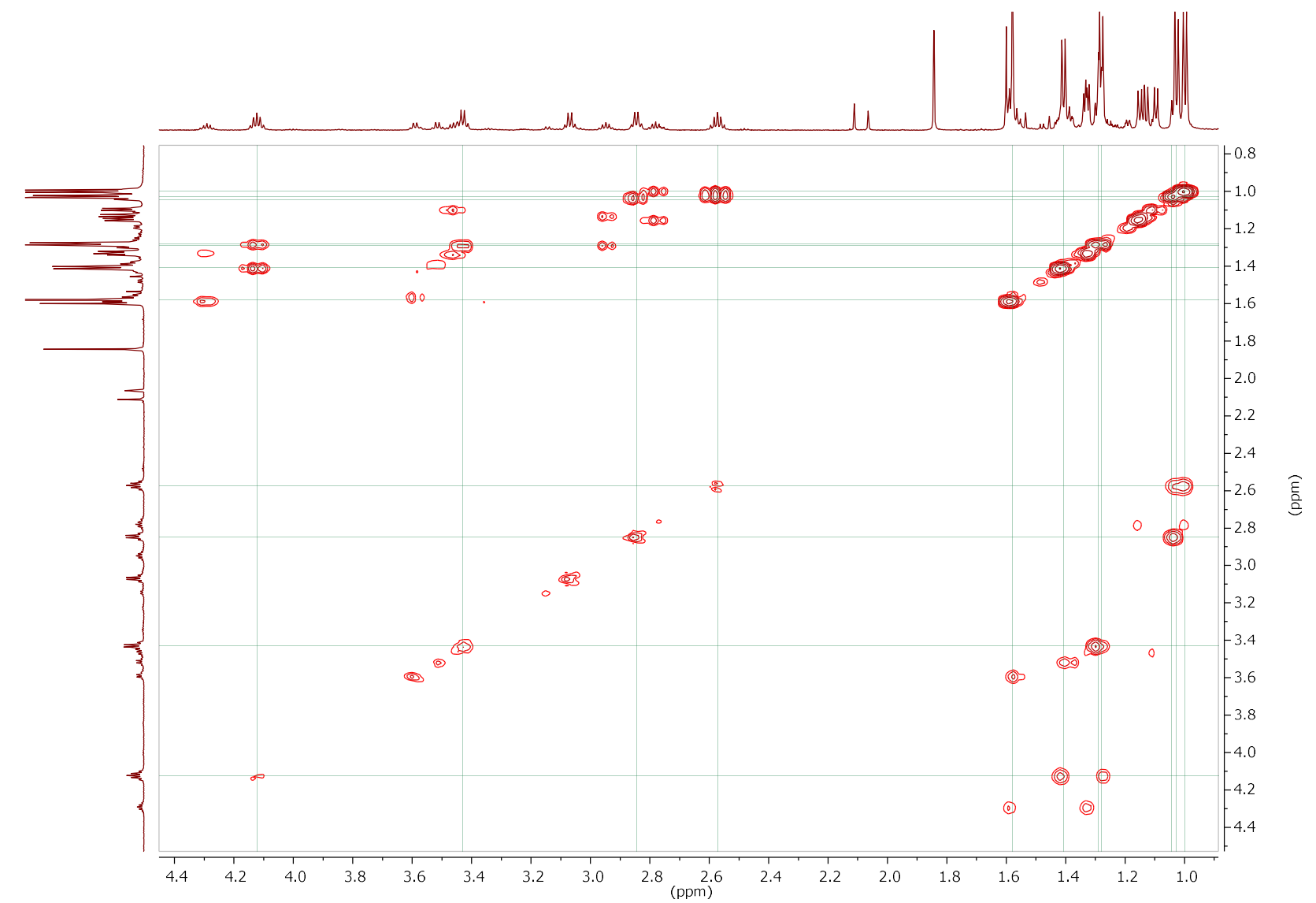

Fig. S6. COSY 2D-NMR spectrum $\left(600 \mathrm{MHz}, \mathrm{C}_{6} \mathrm{D}_{6}, 25^{\circ} \mathrm{C}\right)$ of $\left[\mathrm{L}\left(\mathrm{Et}_{2} \mathrm{~N}\right) \mathrm{AlBi}\right]_{2}$ 9. The correlations of symmetric conformer are marked. 


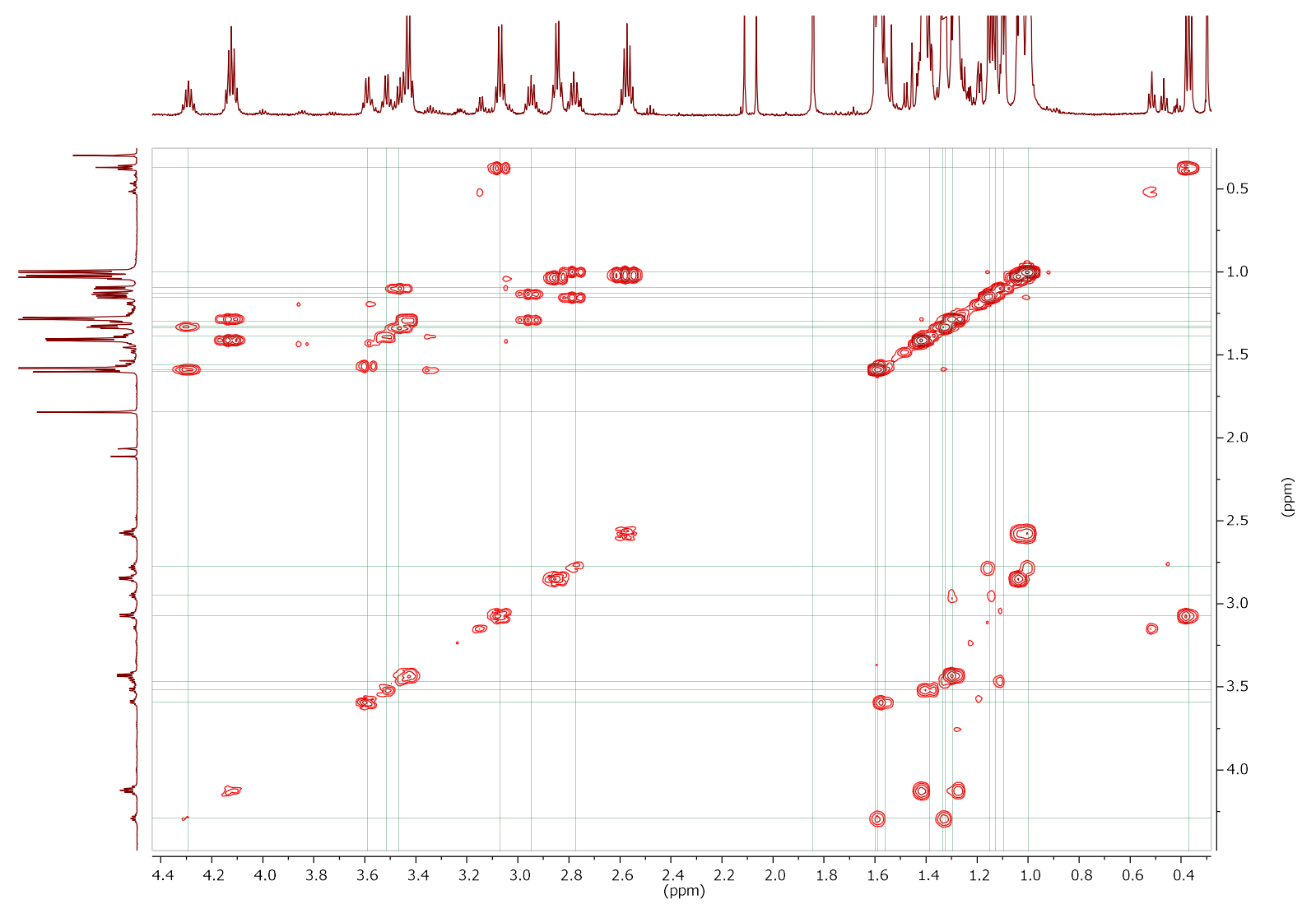

Fig. S7. COSY 2D-NMR spectrum $\left(600 \mathrm{MHz}, \mathrm{C}_{6} \mathrm{D}_{6}, 25^{\circ} \mathrm{C}\right)$ of $\left[\mathrm{L}\left(\mathrm{Et}_{2} \mathrm{~N}\right) \mathrm{AlBi}\right]_{2}$ 9. The correlations of unsymmetric conformer are marked.

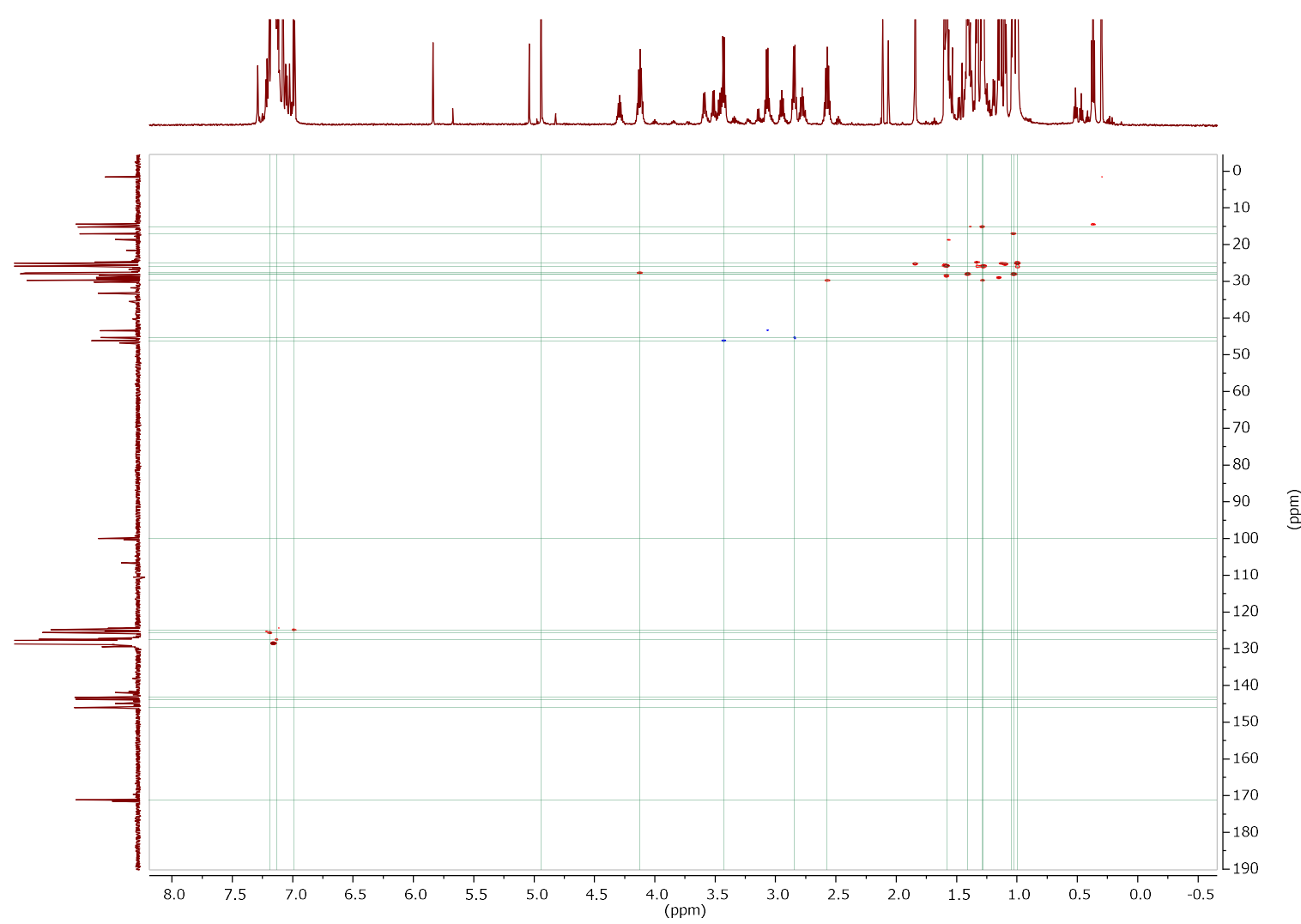

Fig. S8. HSQC 2D-NMR spectrum $\left(600 / 150.9 \mathrm{MHz}, \mathrm{C}_{6} \mathrm{D}_{6}, 25^{\circ} \mathrm{C}\right)$ of $\left[\mathrm{L}\left(\mathrm{Et}_{2} \mathrm{~N}\right) \mathrm{AlBi}\right]_{2}$ 9. The correlations of symmetric conformer are marked. 


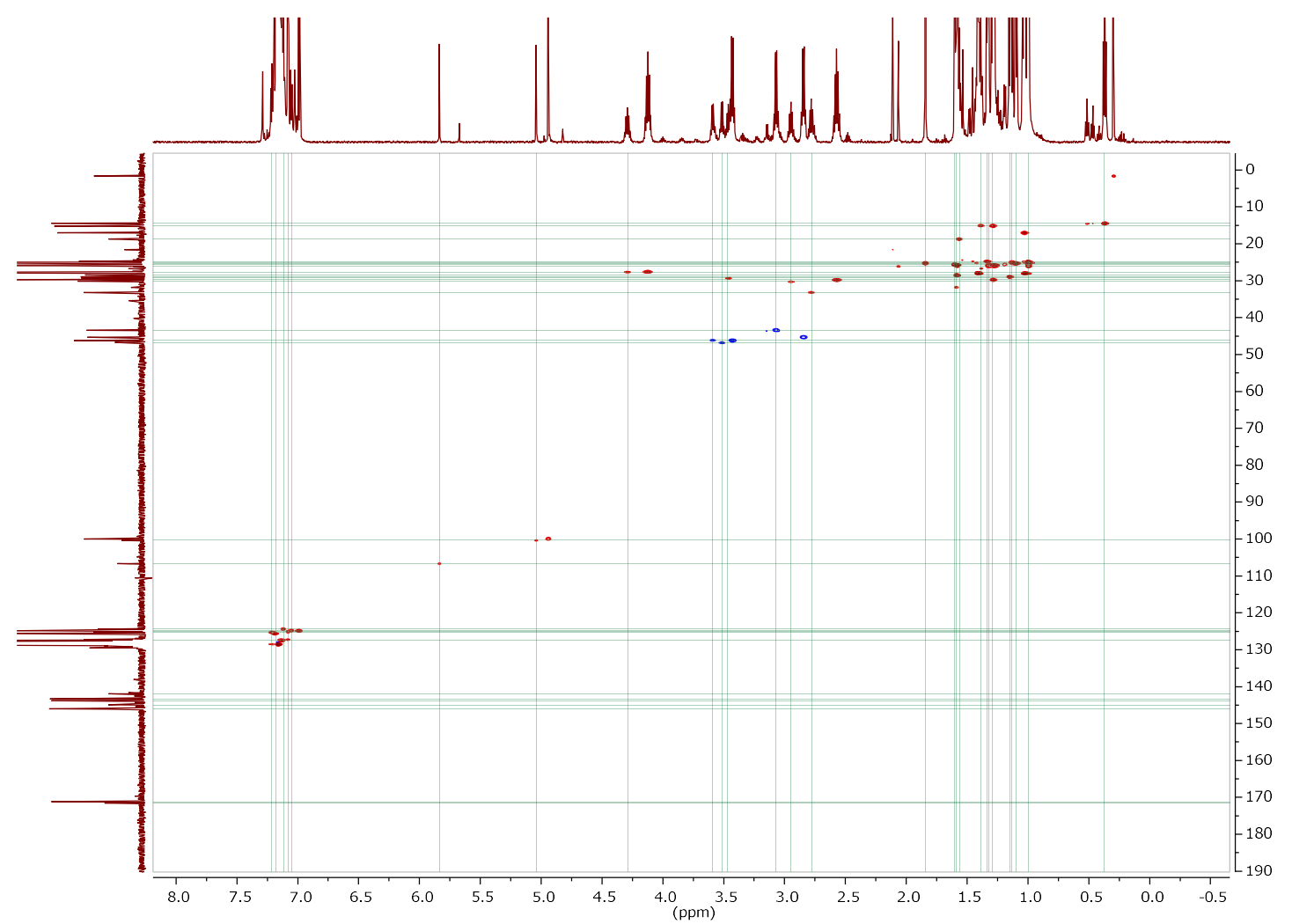

Fig. S9. HSQC 2D-NMR spectrum $\left(600 / 150.9 \mathrm{MHz}, \mathrm{C}_{6} \mathrm{D}_{6}, 25{ }^{\circ} \mathrm{C}\right)$ of $\left.\left[\mathrm{L}_{(\mathrm{Et}} \mathrm{N}\right) \mathrm{AlBi}\right]_{2}$ 9. The correlations of unsymmetric conformer are marked.

The hydrodynamic radius of compound $\left[\mathrm{L}\left(\mathrm{Et}_{2} \mathrm{~N}\right) \mathrm{AlBi}\right]_{2} 9$ was calculated from the sc-XRD data. The molecular volume $\mathrm{V}_{\mathrm{m}}=1634,5 \AA^{3}$ was calculated by the formula $\mathrm{V}_{\mathrm{m}}=\mathrm{V}_{\text {cell }} / \mathrm{Z}$. With respect to 68.7 percent filled space calculated by PLATON ${ }^{1}$ it is minimized to $1122,9 \AA^{3}$. $R_{\min }=6.45 \AA$ is derived from that molecular volume, $V_{\text {mol }}=(4 \pi / 3) R_{\min }{ }^{3} . D_{\max }=18.75 \AA$ is evaluated corresponding to the longest distance from the molecule's barycenter $(16.35 \AA)$ augmented by the two corresponding van der Waals radii $(H=1.2 \AA)$. Therefore, the average hydrodynamic radius $\left(R_{H}\right)$ is $\left(2 r_{\min }+d_{\max }\right) / 4=$ $7.91 \AA$. The $\mathrm{R}_{\mathrm{H}}$ value was then related to the value obtained from ${ }^{1} \mathrm{H}$ DOSY NMR spectrum via the Stokes-Einstein-equation. ${ }^{2-5}$ 

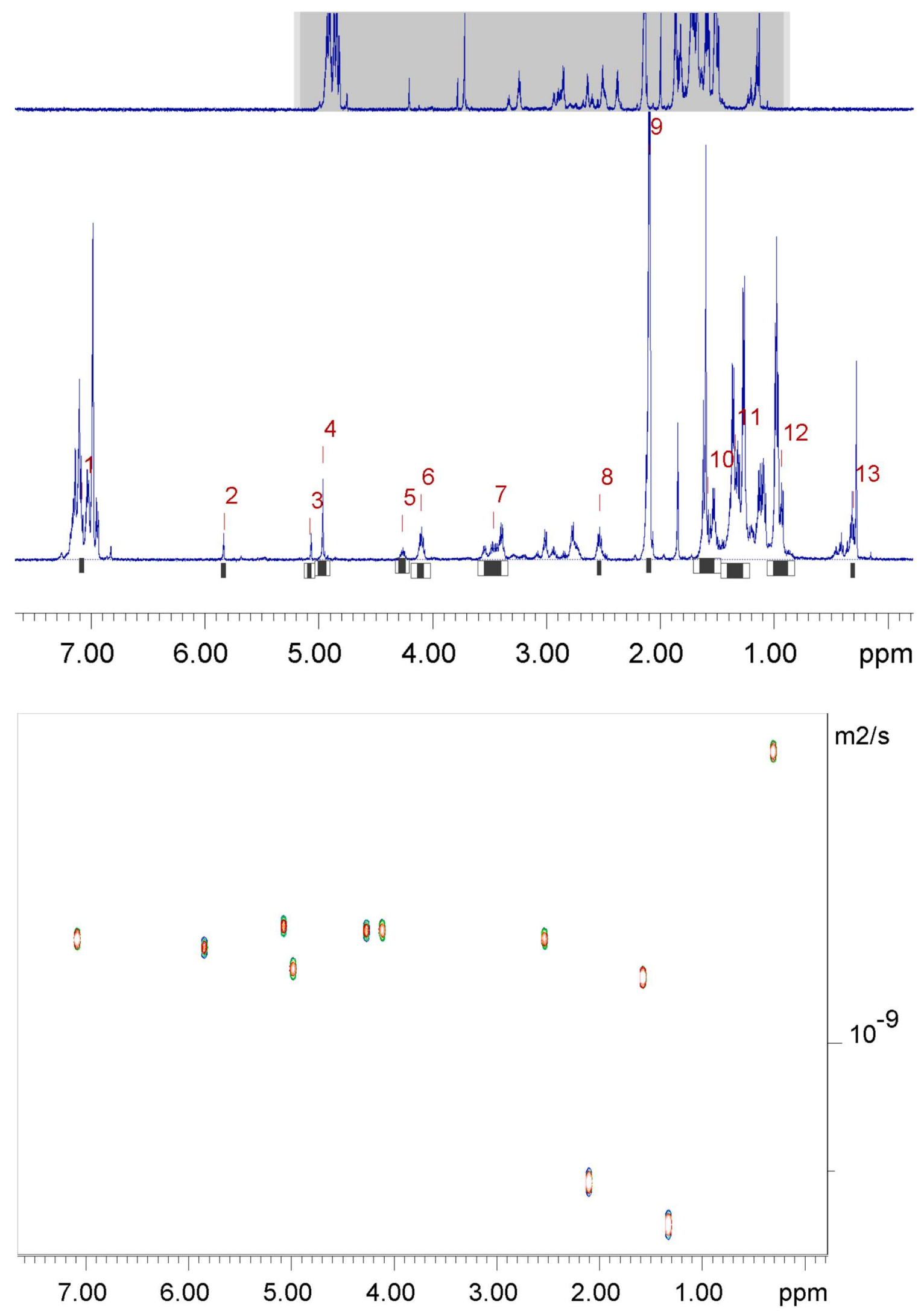

Fig. S10. DOSY NMR of $\left[\mathrm{L}\left(\mathrm{Et}_{2} \mathrm{~N}\right) \mathrm{AlBi}\right]_{2}$ 9. The 3 derivative signals are from left to right toluene, hexane and silicon grease. 
Table S2. DOSY NMR Results of chosen peaks of $\left[\mathrm{L}\left(\mathrm{Et}_{2} \mathrm{~N}\right) \mathrm{AlBi}\right]_{2} 9$.

\begin{tabular}{cccccc}
$\begin{array}{c}\text { Peak } \\
\text { number }\end{array}$ & F2 [ppm] & Io & Error & D [m2/s] & Error \\
\hline \hline 1 & 7.081 & $3.50 \mathrm{e}+09$ & $2.321 \mathrm{e}+06$ & $5.72 \mathrm{e}-10$ & $1.194 \mathrm{e}-12$ \\
2 & 5.834 & $2.96 \mathrm{e}+08$ & $2.278 \mathrm{e}+06$ & $5.90 \mathrm{e}-10$ & $1.153 \mathrm{e}-11$ \\
3 & 5.079 & $3.03 \mathrm{e}+08$ & $3.295 \mathrm{e}+06$ & $5.33 \mathrm{e}-10$ & $1.368 \mathrm{e}-11$ \\
4 & 4.967 & $1.24 \mathrm{e}+09$ & $4.087 \mathrm{e}+06$ & $6.67 \mathrm{e}-10$ & $4.214 \mathrm{e}-12$ \\
5 & 4.266 & $4.46 \mathrm{e}+08$ & $3.015 \mathrm{e}+06$ & $5.48 \mathrm{e}-10$ & $1.047 \mathrm{e}-11$ \\
6 & 4.103 & $1.25 \mathrm{e}+09$ & $2.774 \mathrm{e}+06$ & $5.44 \mathrm{e}-10$ & $3.238 \mathrm{e}-12$ \\
7 & 3.469 & 0.00 & 0.000 & 0.00 & 0.000 \\
8 & 2.533 & $1.22 \mathrm{e}+09$ & $2.339 \mathrm{e}+06$ & $5.62 \mathrm{e}-10$ & $3.315 \mathrm{e}-12$ \\
9 & 2.098 & $2.04 \mathrm{e}+10$ & $3.019 \mathrm{e}+06$ & $2.12 \mathrm{e}-09$ & $7.980 \mathrm{e}-13$ \\
10 & 1.587 & $7.36 \mathrm{e}+09$ & $3.240 \mathrm{e}+06$ & $6.95 \mathrm{e}-10$ & $7.008 \mathrm{e}-13$ \\
11 & 1.341 & $3.21 \mathrm{e}+10$ & $7.596 \mathrm{e}+06$ & $2.68 \mathrm{e}-09$ & $9.447 \mathrm{e}-13$ \\
12 & 0.939 & 0.00 & 0.000 & 0.00 & 0.000 \\
13 & 0.308 & $2.42 \mathrm{e}+09$ & $2.366 \mathrm{e}+06$ & $2.05 \mathrm{e}-10$ & $6.393 \mathrm{e}-13$
\end{tabular}

$B$ values variable. Little delta $=0.001 \mathrm{~s}$. Big delta $=0.03 \mathrm{~s}$. Random error estimation of data: RMS per Spectrum (or trace/plane). Systematic error estimation: worst case per peak scenario. Fit parameter Error estimation method: from fit using calculated y uncertainties. Confidence level: $95 \%$. Used integrals: area integral.

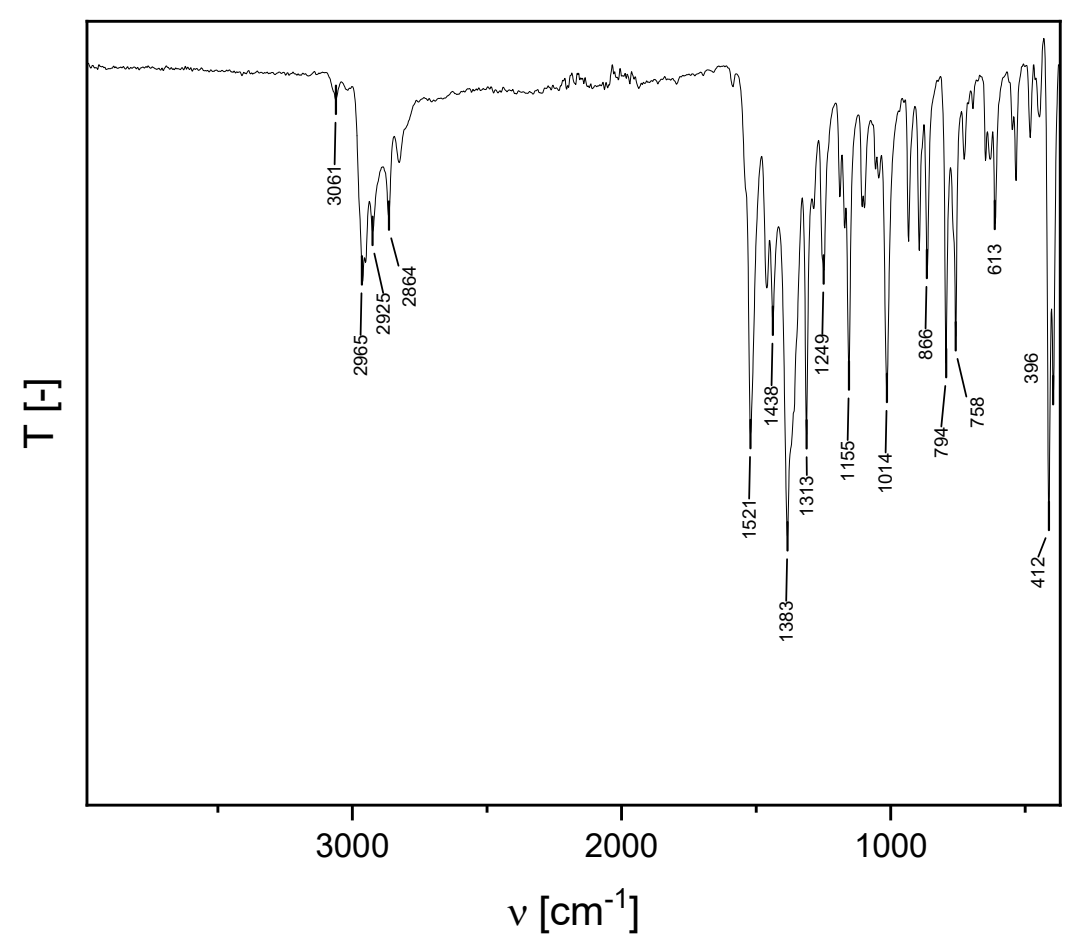

Fig. S11. ATR-IR spectrum of $\left[\mathrm{L}\left(\mathrm{Et}_{2} \mathrm{~N}\right) \mathrm{AlBi}\right]_{2} 9$. 


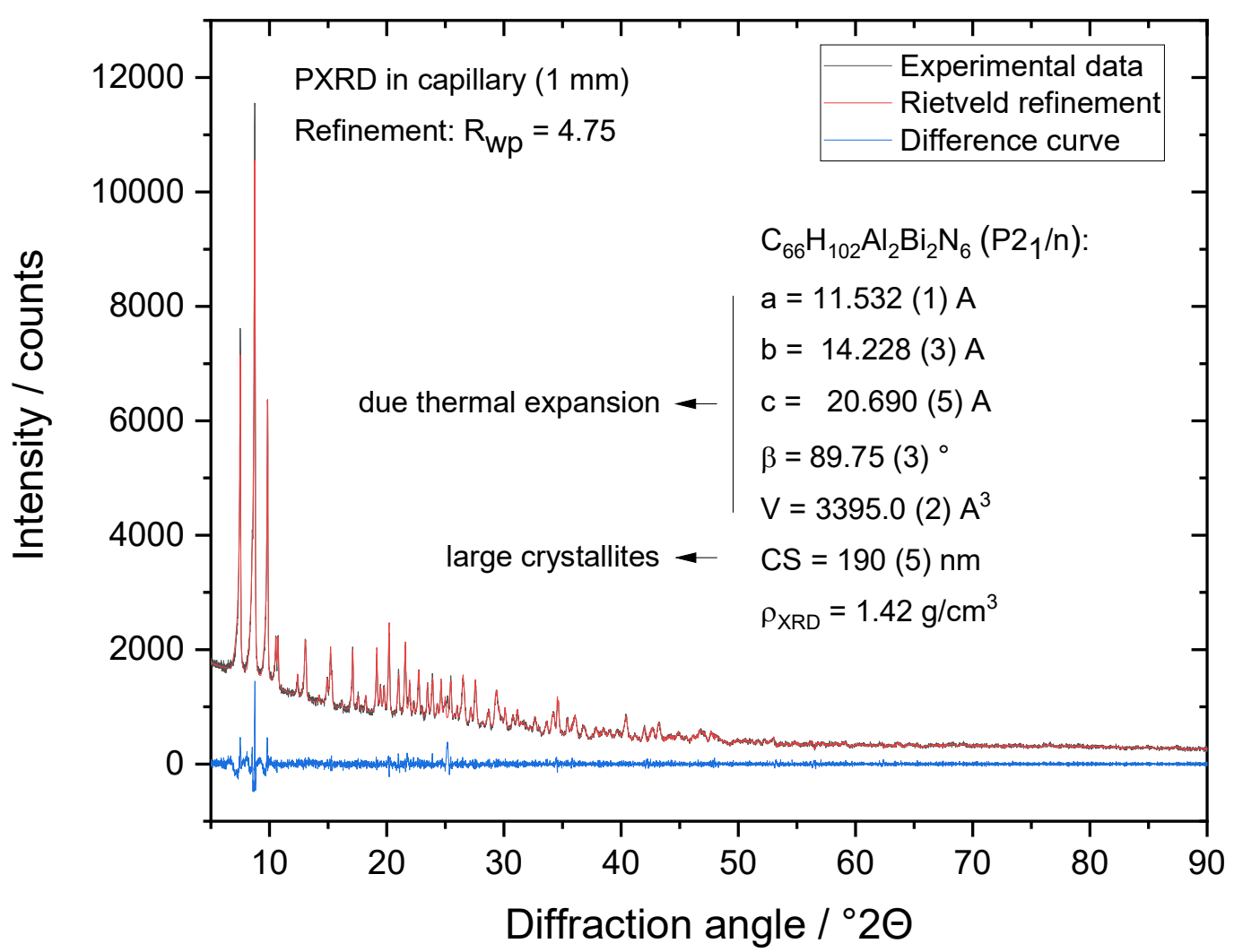

Fig. S12. Powder-XRD of $\left[\mathrm{L}\left(\mathrm{Et}_{2} \mathrm{~N}\right) \mathrm{AlBi}\right]_{2} 9$ and Rietveld refinement on basis of the SC-XRD.

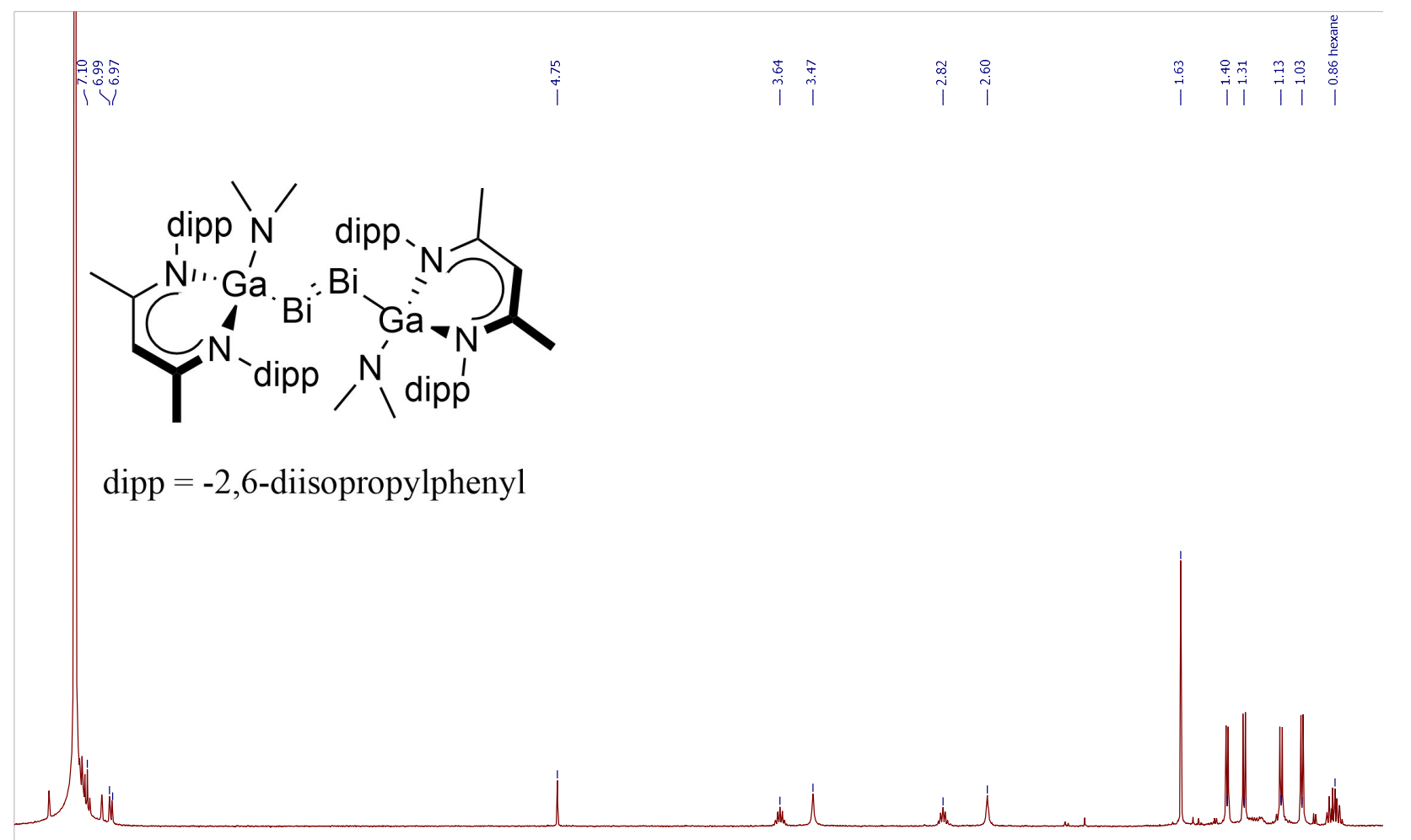

\begin{tabular}{lllllllllllllllllllllllllllllllllllllllllllllllll}
\hline 7.4 & 7.2 & 7.0 & 6.8 & 6.6 & 6.4 & 6.2 & 6.0 & 5.8 & 5.6 & 5.4 & 5.2 & 5.0 & 4.8 & 4.6 & 4.4 & 4.2 & 4.0 & 3.8 & 3.6 & 1 & 1.4 & 3.2 & 3.0 & 2.8 & 2.6 & 2.4 & 2.2 & 2.0 & 1.8 & 1.6 & 1.4 & 1.2 & 1.0 & 0.8
\end{tabular}

Fig. S13. ${ }^{1} \mathrm{H}$ NMR spectrum $\left(600 \mathrm{MHz}, \mathrm{C}_{6} \mathrm{D}_{6}, 25^{\circ} \mathrm{C}\right)$ of $\left[\mathrm{L}\left(\mathrm{Me}_{2} \mathrm{~N}\right) \mathrm{GaBi}\right]_{2} \mathbf{1 0}$. 


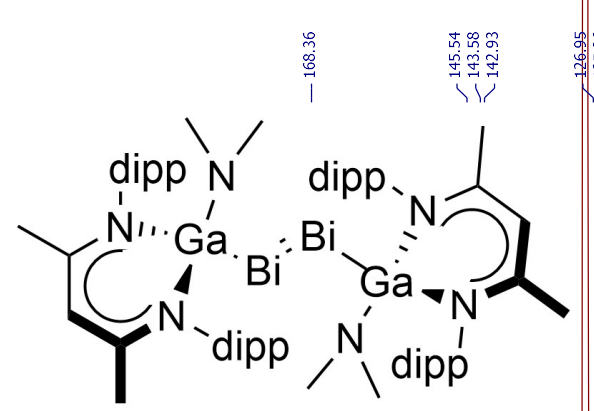

$\operatorname{dipp}=-2,6$-diisopropylphenyl

ig. S14. ${ }^{13} \mathrm{C}$ NMR spectrum $\left(150.9 \mathrm{MHz}, \mathrm{C}_{6} \mathrm{D}_{6}, 25^{\circ} \mathrm{C}\right)$ of $\left[\mathrm{L}\left(\mathrm{Me}_{2} \mathrm{~N}\right) \mathrm{GaBi}\right]_{2} \mathbf{1 0}$.

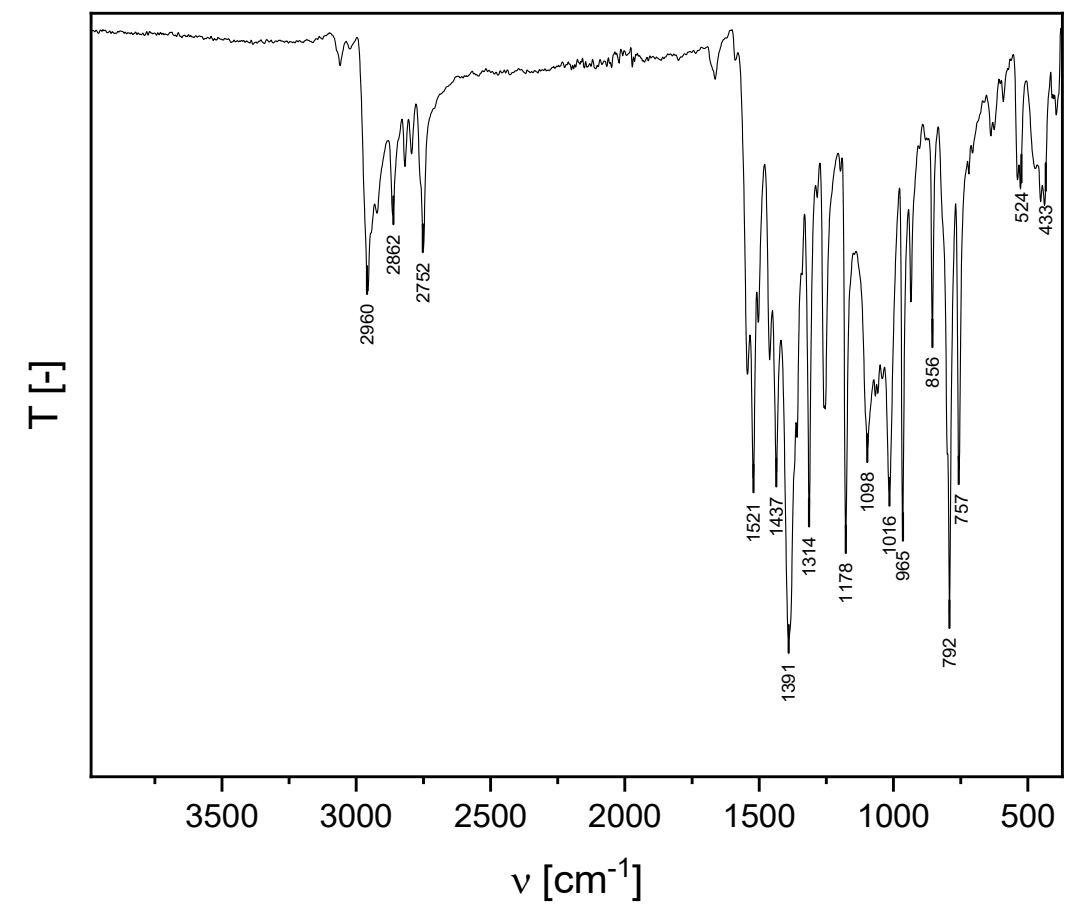

Fig. S15. ATR-IR spectrum of $\left[\mathrm{L}\left(\mathrm{Me}_{2} \mathrm{~N}\right) \mathrm{GaBi}\right]_{2} \mathbf{1 0}$. 


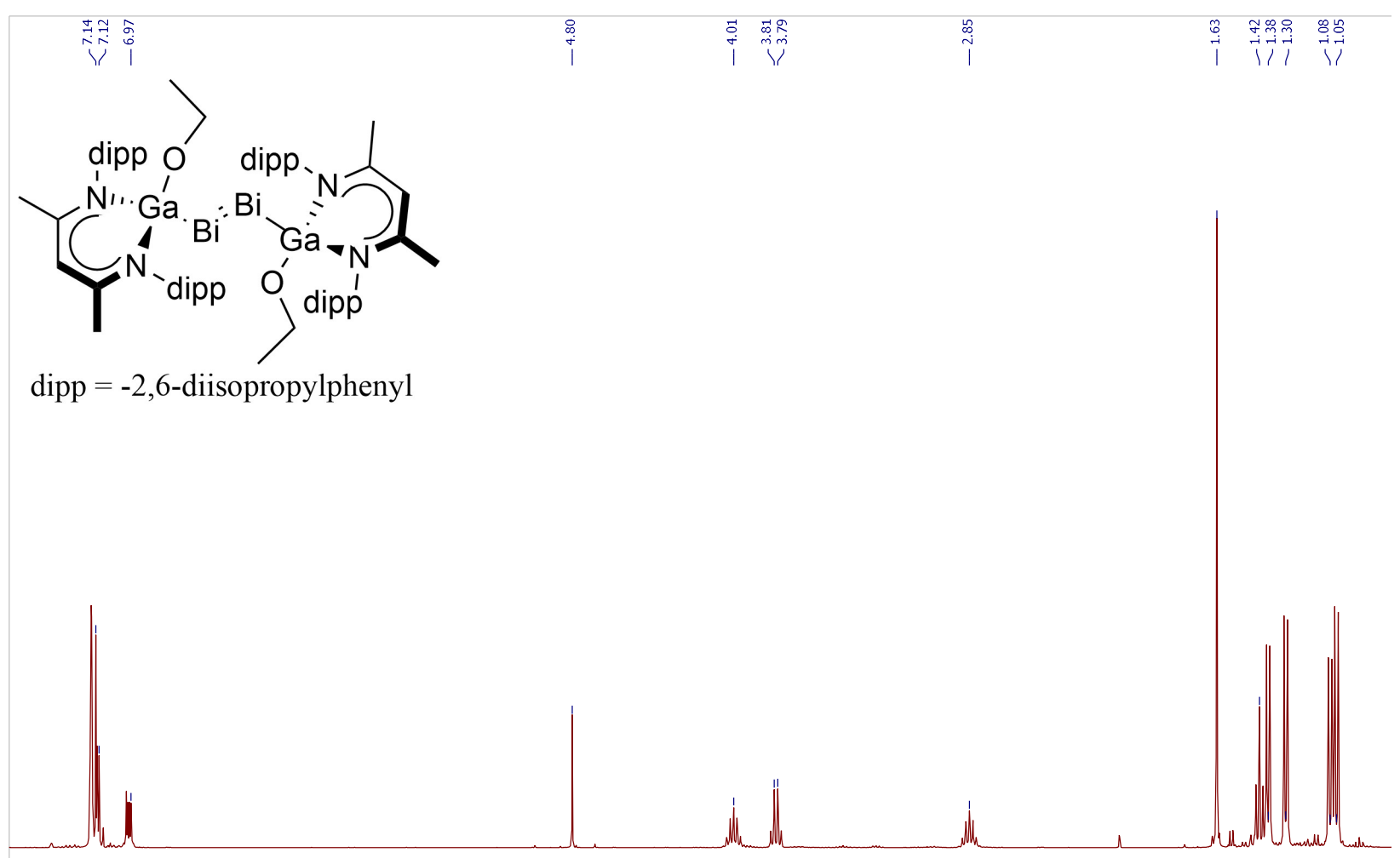

$\begin{array}{llllllllllllllllllllllllllllllllllllllllllllll}7.4 & 7.2 & 7.0 & 6.8 & 6.6 & 6.4 & 6.2 & 6.0 & 5.8 & 5.6 & 5.4 & 5.2 & 5.0 & 4.8 & 4.6 & 4.4 & 4.2 & 4.0 & 3.8 & 3.6 & 3.4 & 3.2 & 3.0 & 2.8 & 2.6 & 2.4 & 2.2 & 2.0 & 1.8 & 1.6 & 1.4 & 1.2 & 1.0 & 0\end{array}$

Fig. S16. ${ }^{1} \mathrm{H}$ NMR spectrum $\left(300 \mathrm{MHz}, \mathrm{C}_{6} \mathrm{D}_{6}, 25^{\circ} \mathrm{C}\right)$ of $[\mathrm{L}(\mathrm{OEt}) \mathrm{GaBi}]_{2} 11$.
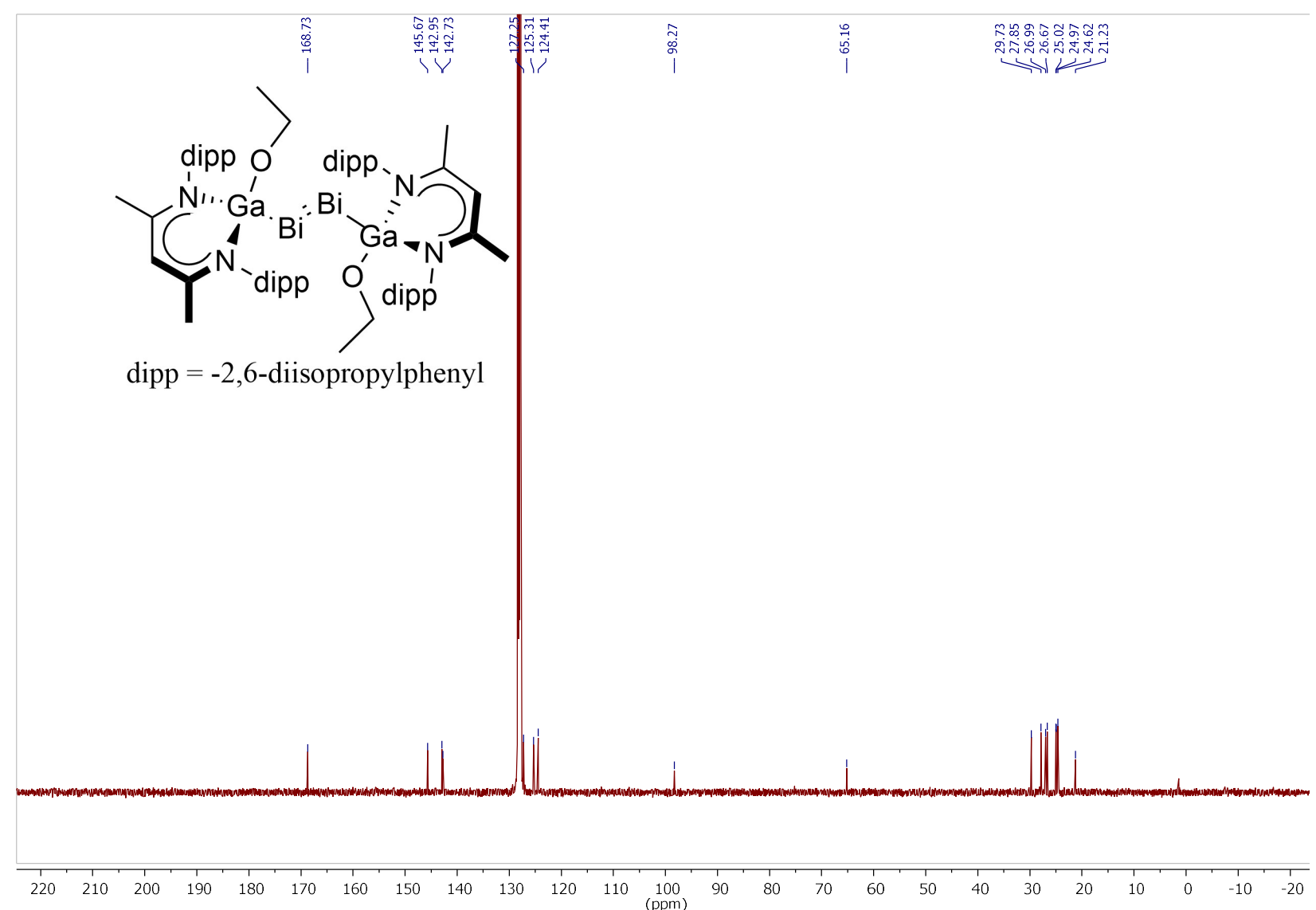

Fig. S17. ${ }^{13} \mathrm{C}$ NMR spectrum $\left(150.9 \mathrm{MHz}, \mathrm{C}_{6} \mathrm{D}_{6}, 25^{\circ} \mathrm{C}\right)$ of $[\mathrm{L}(\mathrm{EtO}) \mathrm{GaBi}]_{2} \mathbf{1 1}$. 


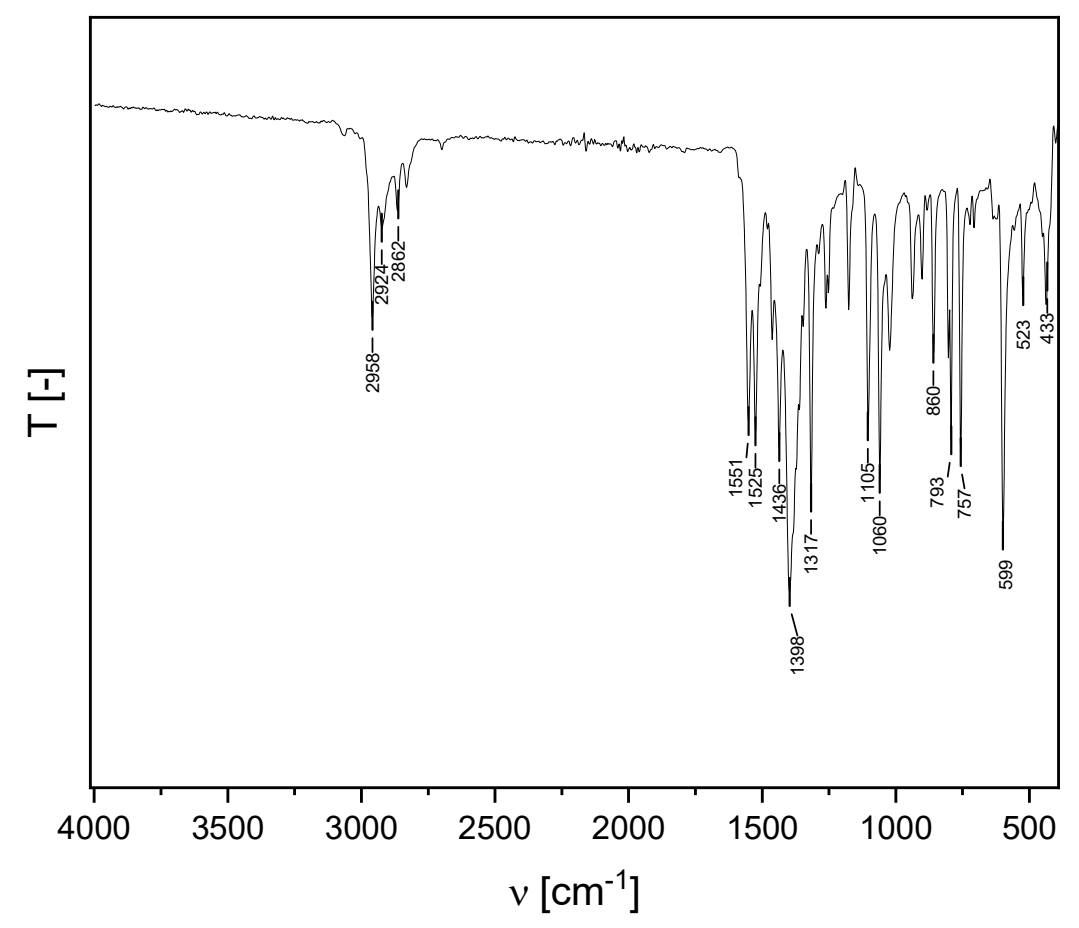

Fig. S 18. ATR-IR spectrum of $[\mathrm{L}(\mathrm{EtO}) \mathrm{GaBi}]_{2} 11$.

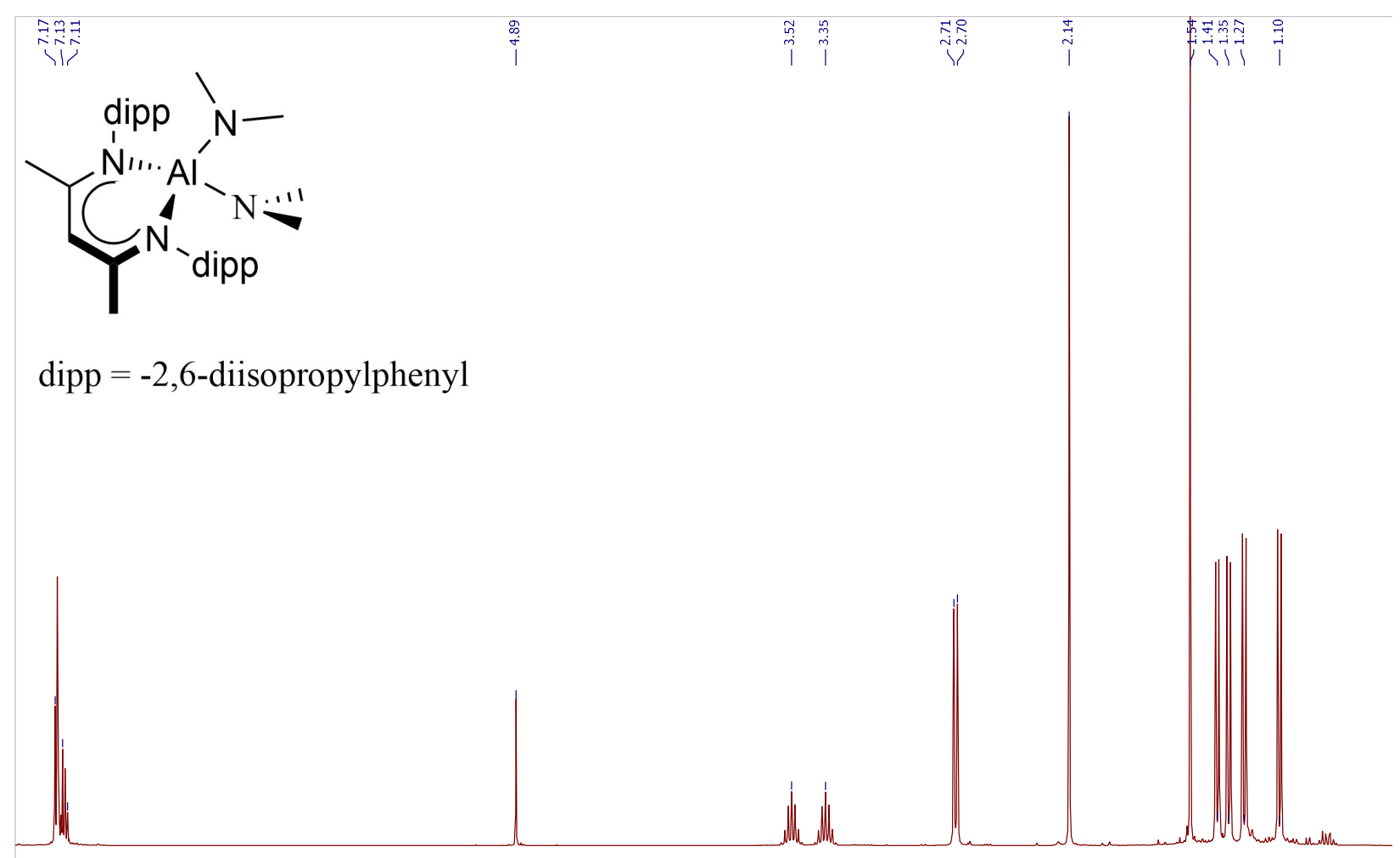

$\begin{array}{llllllllllllllllllllllllllllllllllllllll}7.2 & 7.0 & 6.8 & 6.6 & 6.4 & 6.2 & 6.0 & 5.8 & 5.6 & 5.4 & 5.2 & 5.0 & 4.8 & 4.6 & 4.4 & 4.2 & 4.0 & 3.8 & 3.6 & 3.4 & 3.2 & 3.0 & 2.8 & 2.6 & 2.4 & 2.2 & 2.0 & 1.8 & 1.6 & 1.4 & 1.2 & 1.0 & 0.8 & 0.6\end{array}$

Fig. S19. ${ }^{1} \mathrm{H}$ NMR spectrum $\left(400 \mathrm{MHz}, \mathrm{C}_{6} \mathrm{D}_{6}, 25^{\circ} \mathrm{C}\right)$ of $\mathrm{LAl}\left(\mathrm{NMe}_{2}\right)_{2} 12$. 


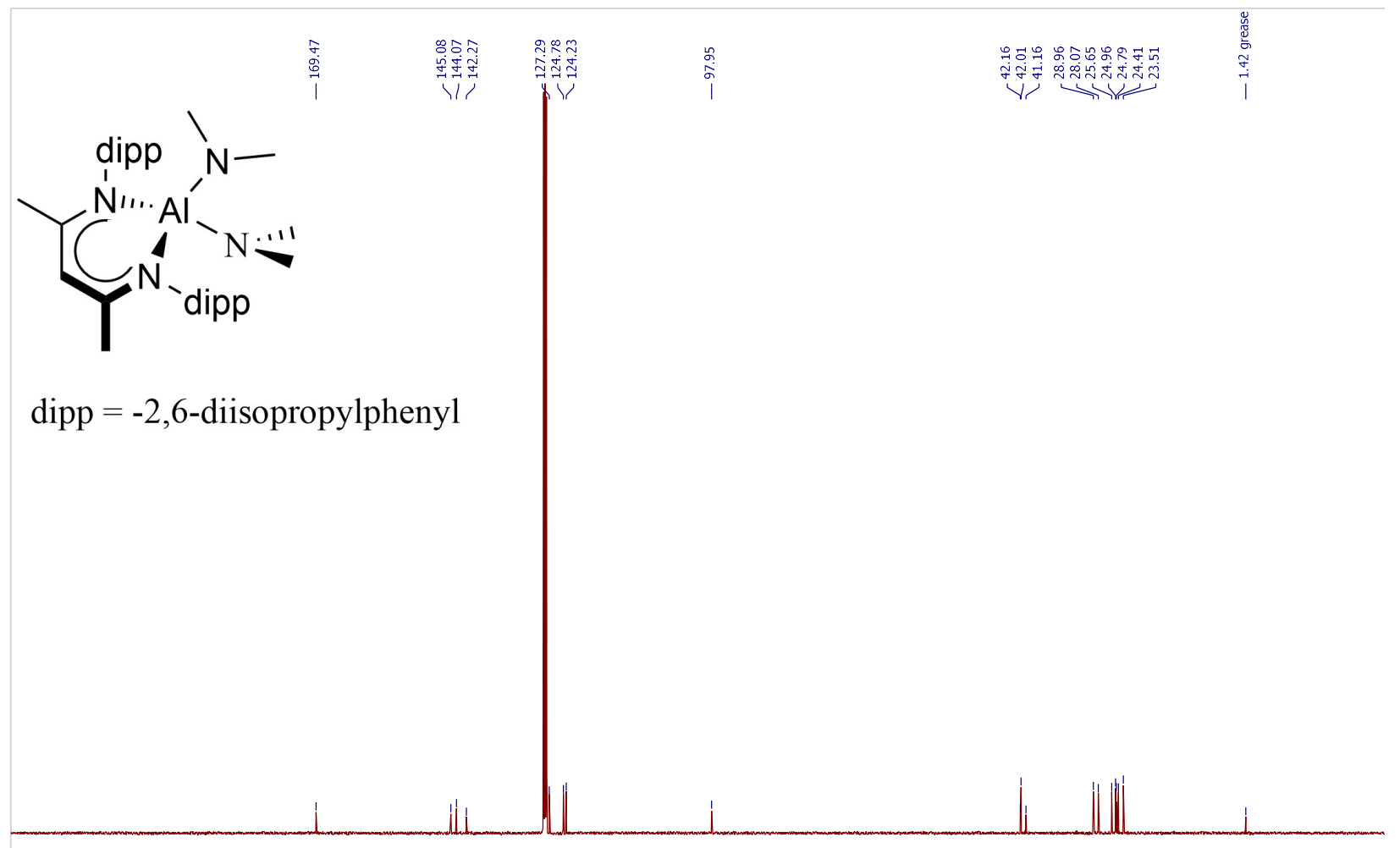

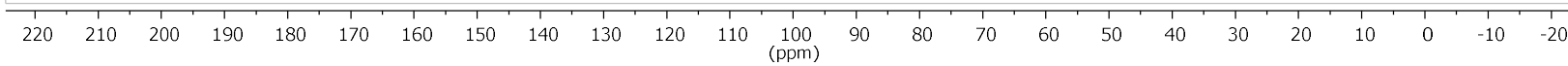

Fig. S20. ${ }^{13} \mathrm{C}$ NMR spectrum $\left(100.7 \mathrm{MHz}, \mathrm{C}_{6} \mathrm{D}_{6}, 25^{\circ} \mathrm{C}\right)$ of $\mathrm{LAl}\left(\mathrm{NMe}_{2}\right)_{2} 12$.

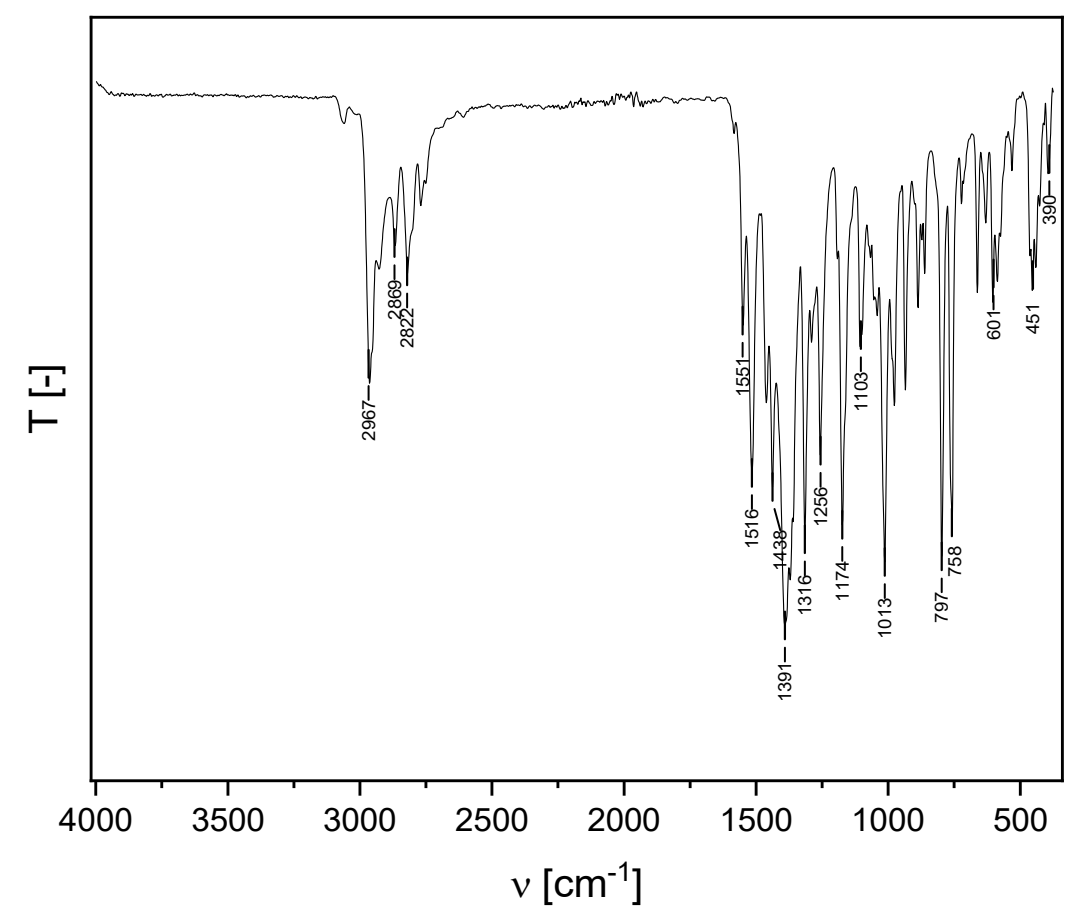

Fig. S21. ATR-IR spectrum of $\mathrm{LAl}\left(\mathrm{NMe}_{2}\right)_{2} 12$. 

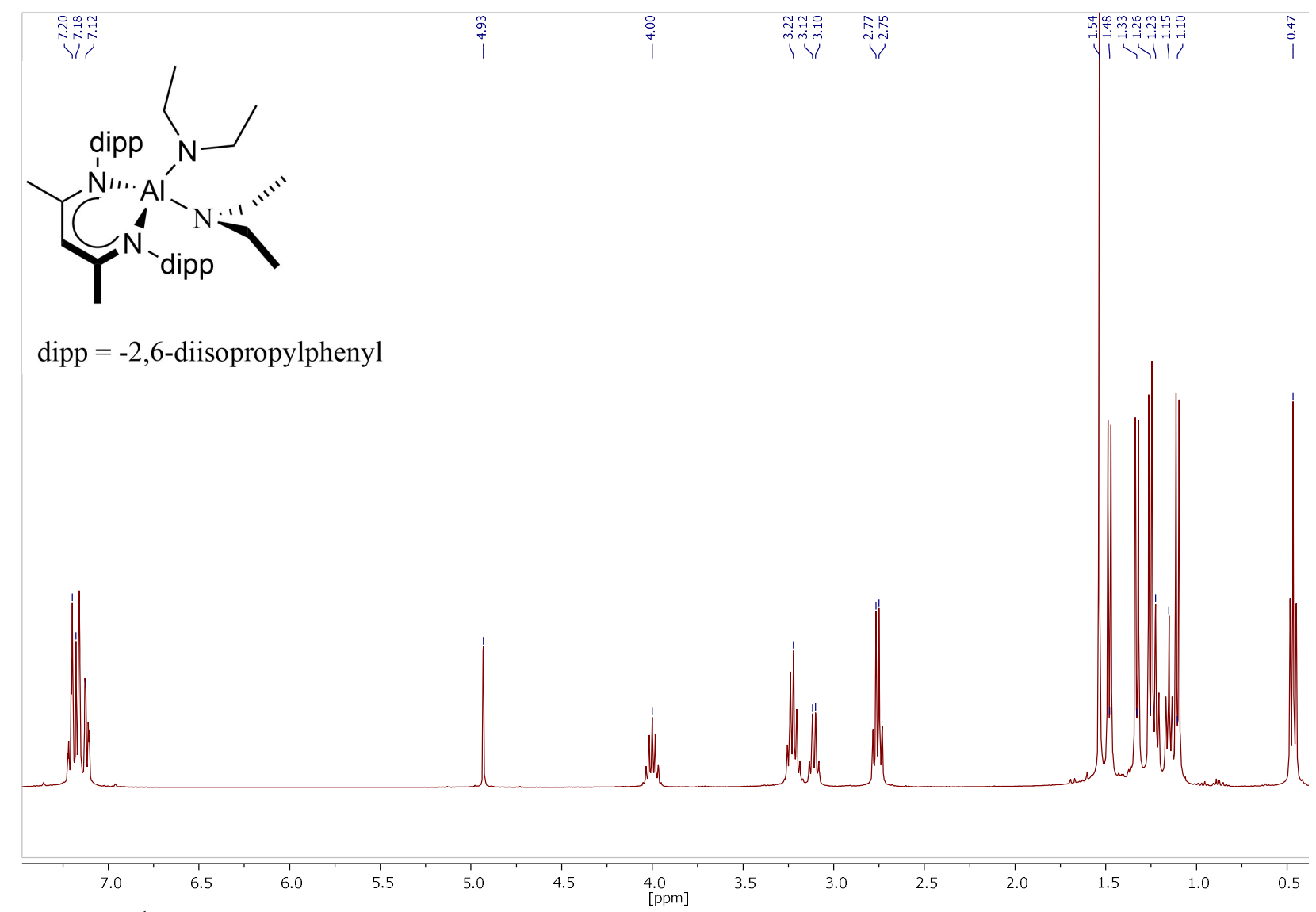

Fig. S22. ${ }^{1} \mathrm{H}$ NMR spectrum $\left(400 \mathrm{MHz}, \mathrm{C}_{6} \mathrm{D}_{6}, 25^{\circ} \mathrm{C}\right)$ of $\mathrm{LAl}_{\left(\mathrm{NEt}_{2}\right)_{2}} \mathbf{1 3}$.

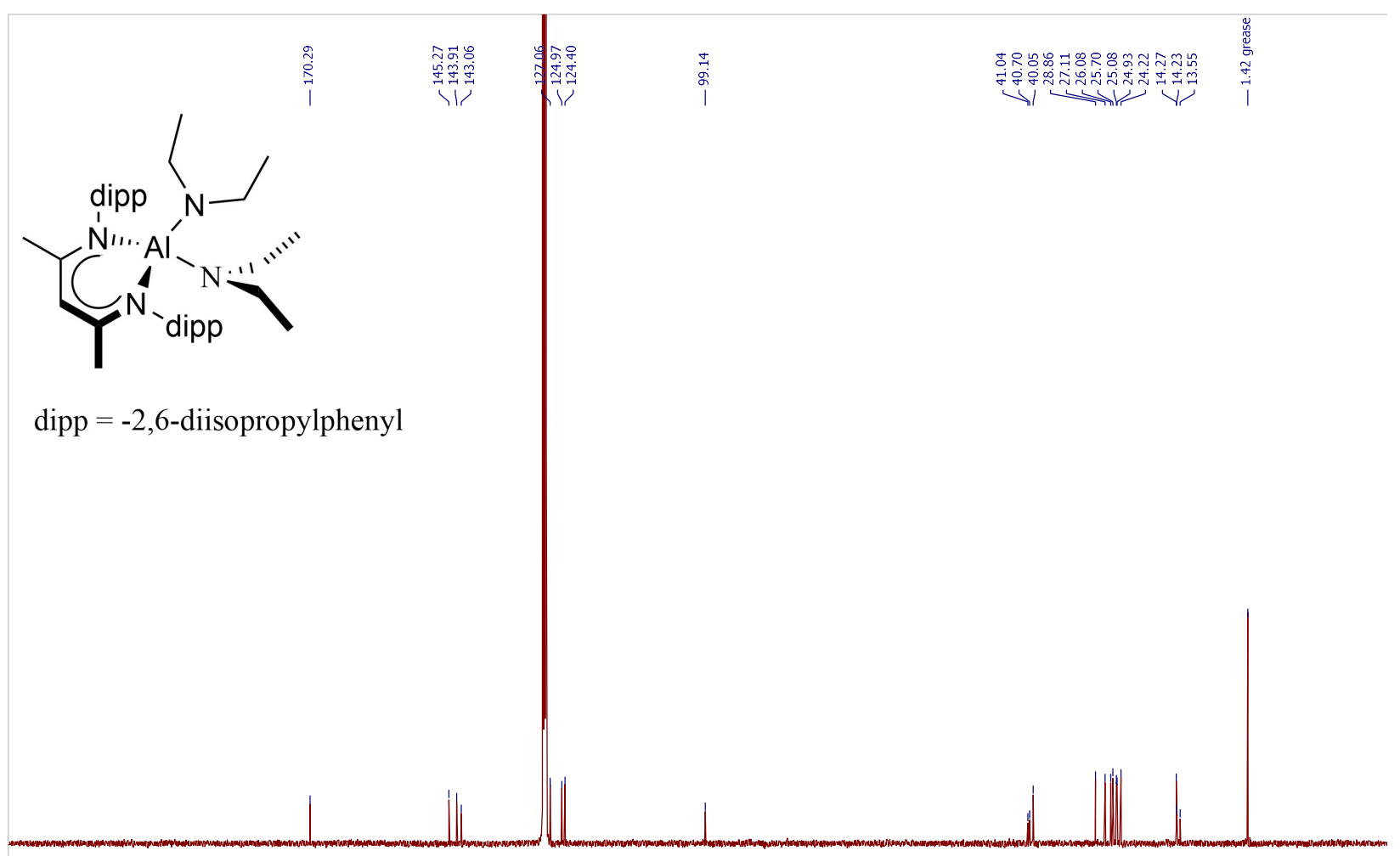

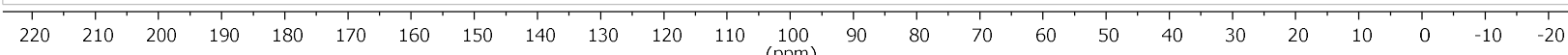

Fig. S23. ${ }^{13} \mathrm{C}$ NMR spectrum $\left(100.7 \mathrm{MHz}, \mathrm{C}_{6} \mathrm{D}_{6}, 25^{\circ} \mathrm{C}\right)$ of $\mathrm{LAl}_{\left(\mathrm{NEt}_{2}\right)_{2}} 13$. 


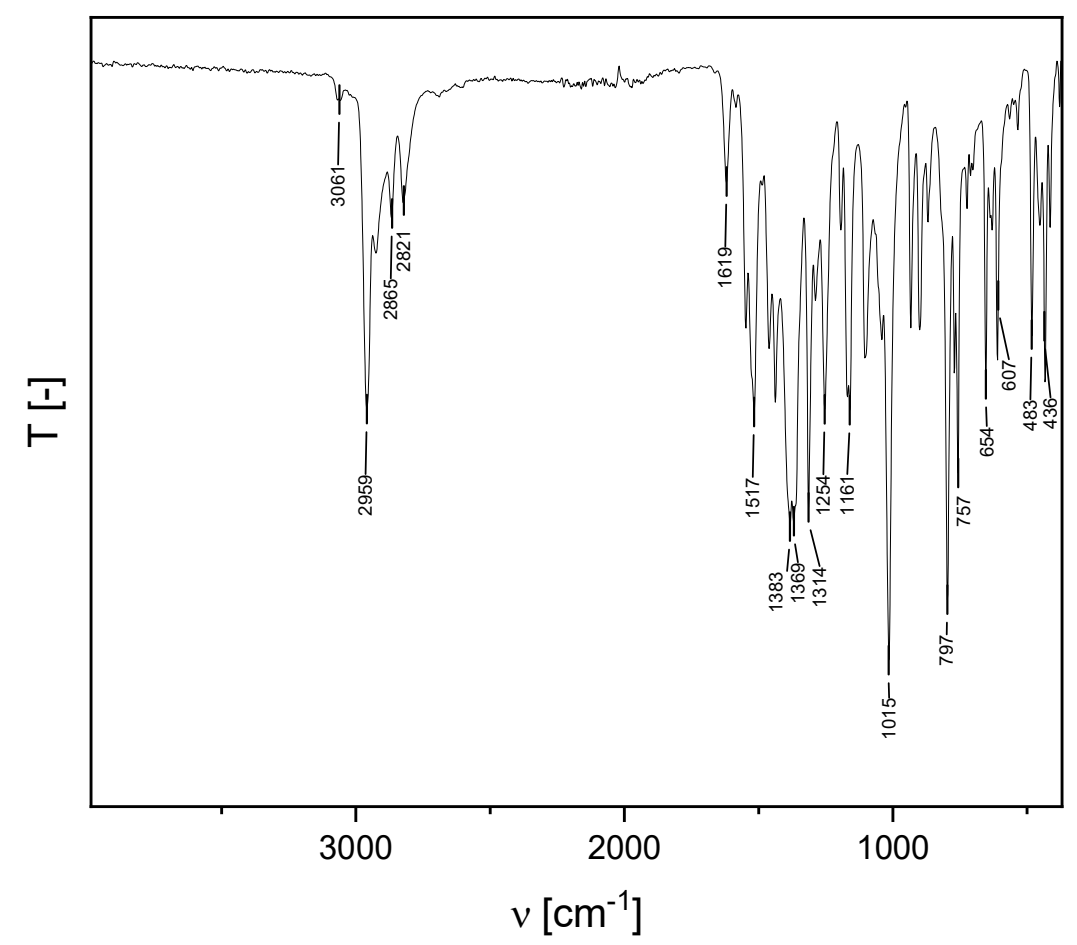

Fig. S24. ATR-IR spectrum of LAl( $\left(\mathrm{NEt}_{2}\right)_{2} 13$.

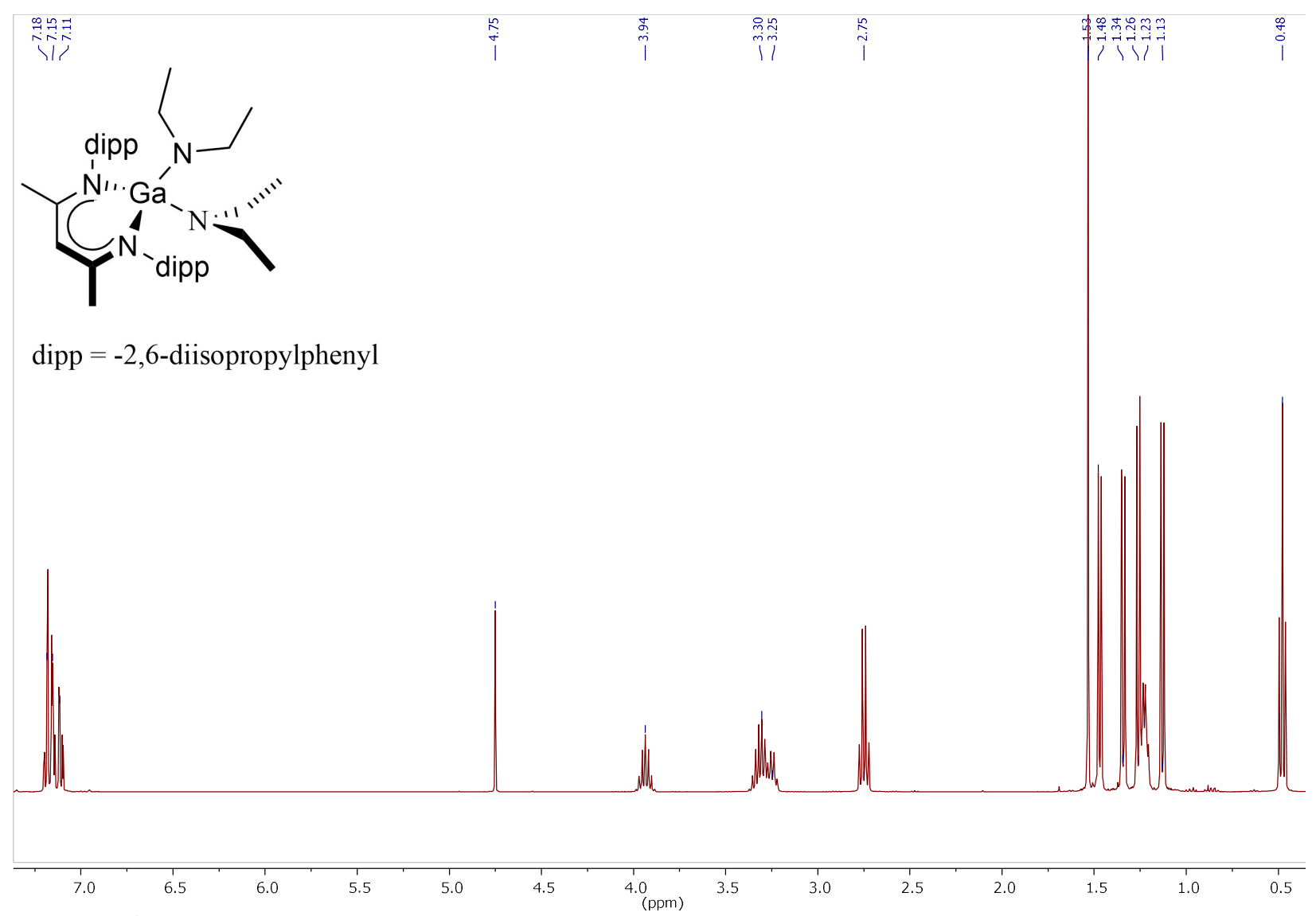

Fig. S25. ${ }^{1} \mathrm{H}$ NMR spectrum ( $\left.400 \mathrm{MHz}, \mathrm{C}_{6} \mathrm{D}_{6}, 25^{\circ} \mathrm{C}\right)$ of $\mathrm{LGa}\left(\mathrm{NEt}_{2}\right)_{2} 14$. 

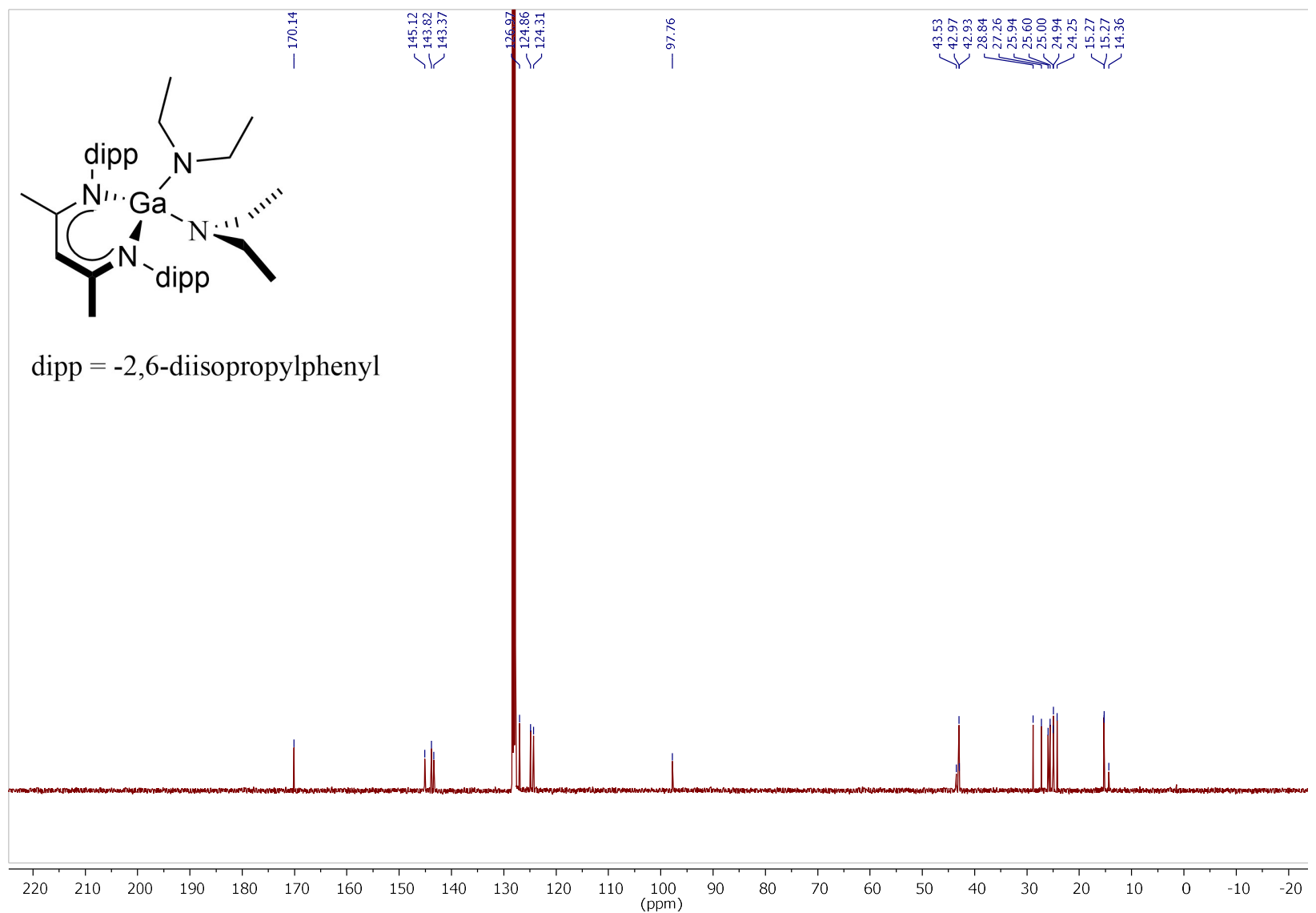

Fig. S26. ${ }^{13} \mathrm{C}$ NMR spectrum $\left(100.7 \mathrm{MHz}, \mathrm{C}_{6} \mathrm{D}_{6}, 25^{\circ} \mathrm{C}\right)$ of $\mathrm{LGa}\left(\mathrm{NEt}_{2}\right)_{2} 14$.

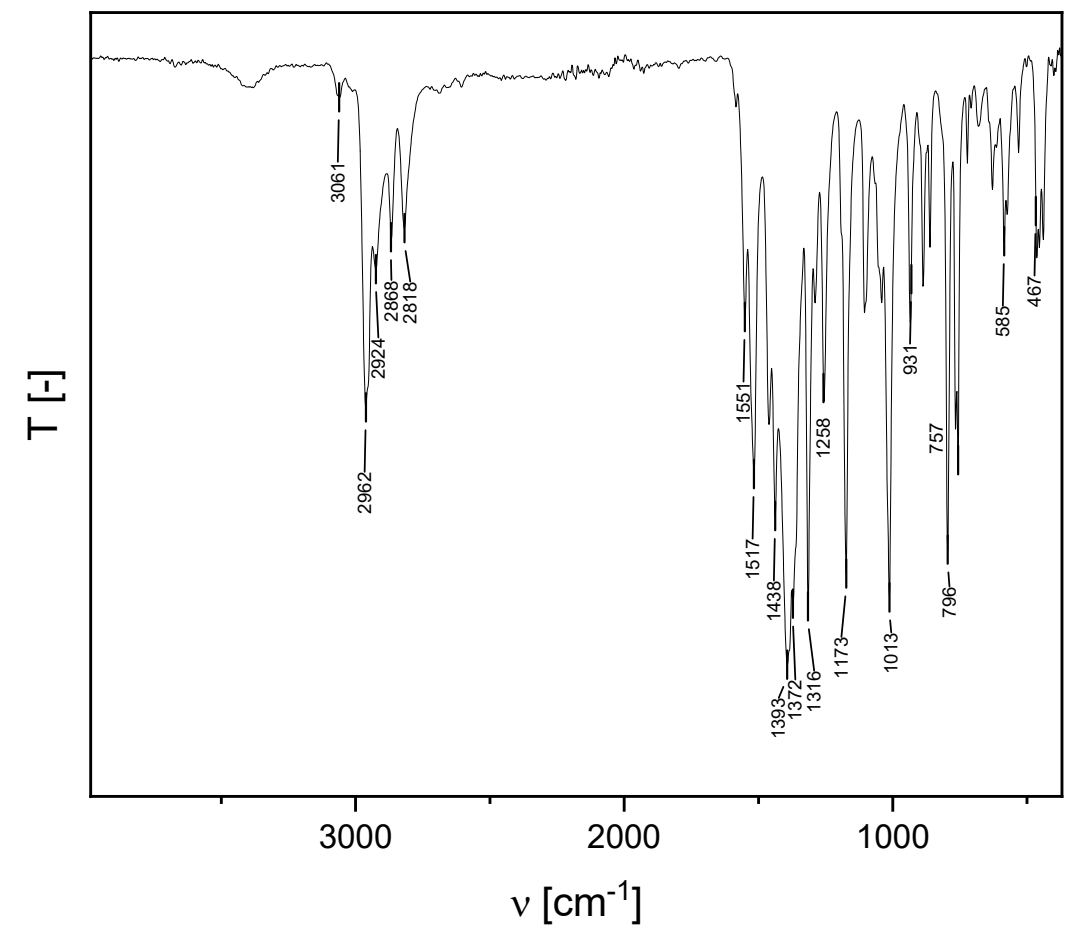

Fig. S27. ATR-IR spectrum of $\mathrm{LGa}\left(\mathrm{NEt}_{2}\right)_{2} 14$. 


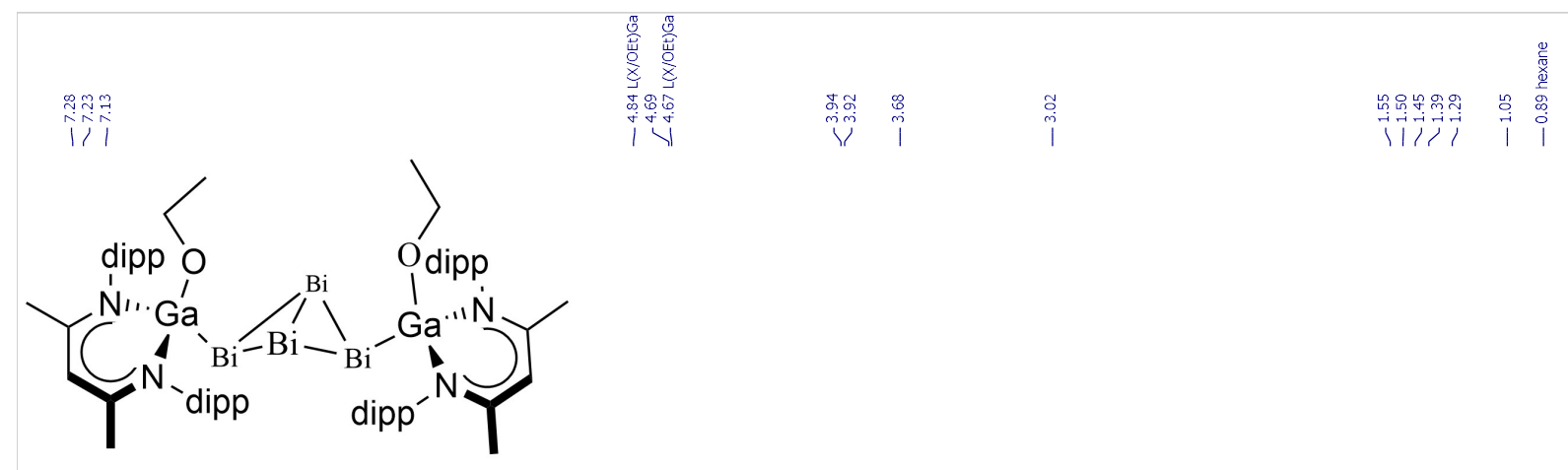

$\operatorname{dipp}=-2,6$-diisopropylphenyl

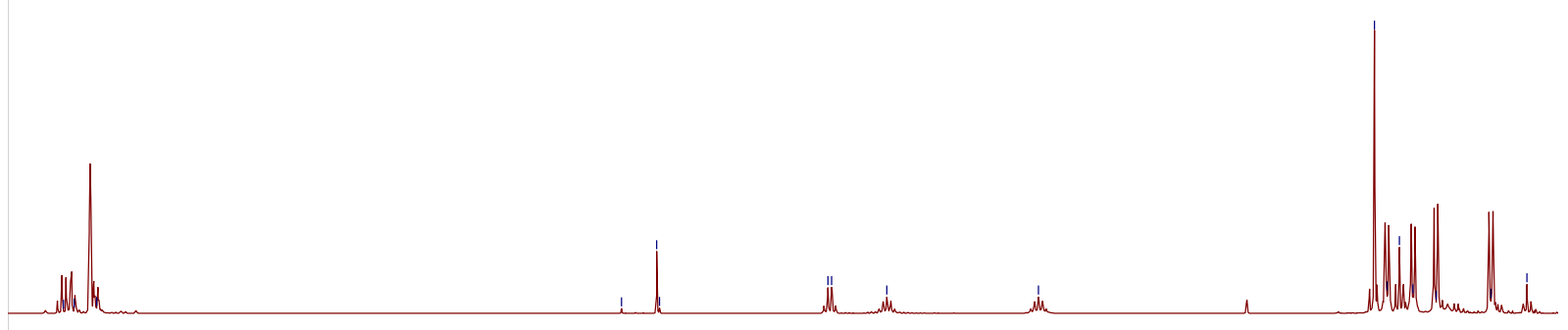

$\begin{array}{lllllllllllllllllllllllllllllllllllllllllll}7.4 & 7.2 & 7.0 & 6.8 & 6.6 & 6.4 & 6.2 & 6.0 & 5.8 & 5.6 & 5.4 & 5.2 & 5.0 & 4.8 & 4.6 & 4.4 & 4.2 & 4.0 & 3.8 & 3.6 & 3.4 & 3.2 & 3.0 & 2.8 & 2.6 & 2.4 & 1 & 2.2 & 2.0 & 1.8 & 1.6 & 1.4 & 1.2 & 1.0 & 0.4\end{array}$

Fig. S28. ${ }^{1} \mathrm{H}$ NMR spectrum $\left(300 \mathrm{MHz}, \mathrm{C}_{6} \mathrm{D}_{6}, 25^{\circ} \mathrm{C}\right)$ of $\left[\{\mathrm{L}(\mathrm{EtO}) \mathrm{Ga}\}_{2}-\mu, \eta^{1: 1}-\mathrm{Bi}_{4}\right] 15$. Impurity likely $\left[\{\mathrm{L}(\mathrm{EtO}) \mathrm{Ga}\}_{1}\left[\{\mathrm{~L}(\mathrm{I}) \mathrm{Ga}\}_{1}-\mu, \eta^{1: 1}-\mathrm{Bi}_{4}\right]\right.$, circa $5 \%$. Recrystallization did not improve the ratio and scXRD suggest the incorporation in the cell.

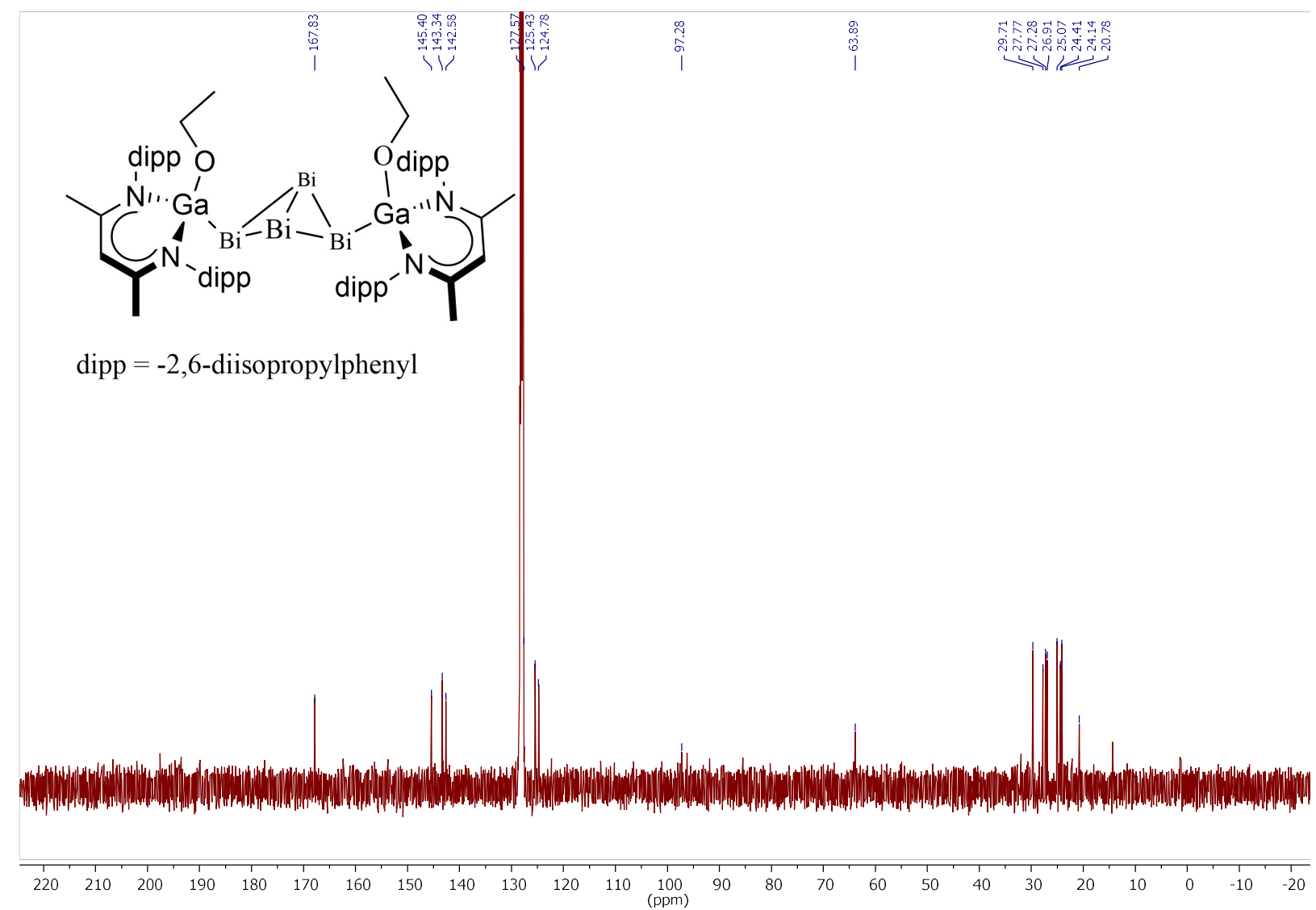

Fig. S29. ${ }^{13} \mathrm{C}$ NMR spectrum $\left(150.9 \mathrm{MHz}, \mathrm{C}_{6} \mathrm{D}_{6}, 25^{\circ} \mathrm{C}\right)$ of $\left[\{\mathrm{L}(\mathrm{EtO}) \mathrm{Ga}\}_{2}-\mu, \eta^{1: 1}-\mathrm{Bi}_{4}\right] 15$. 


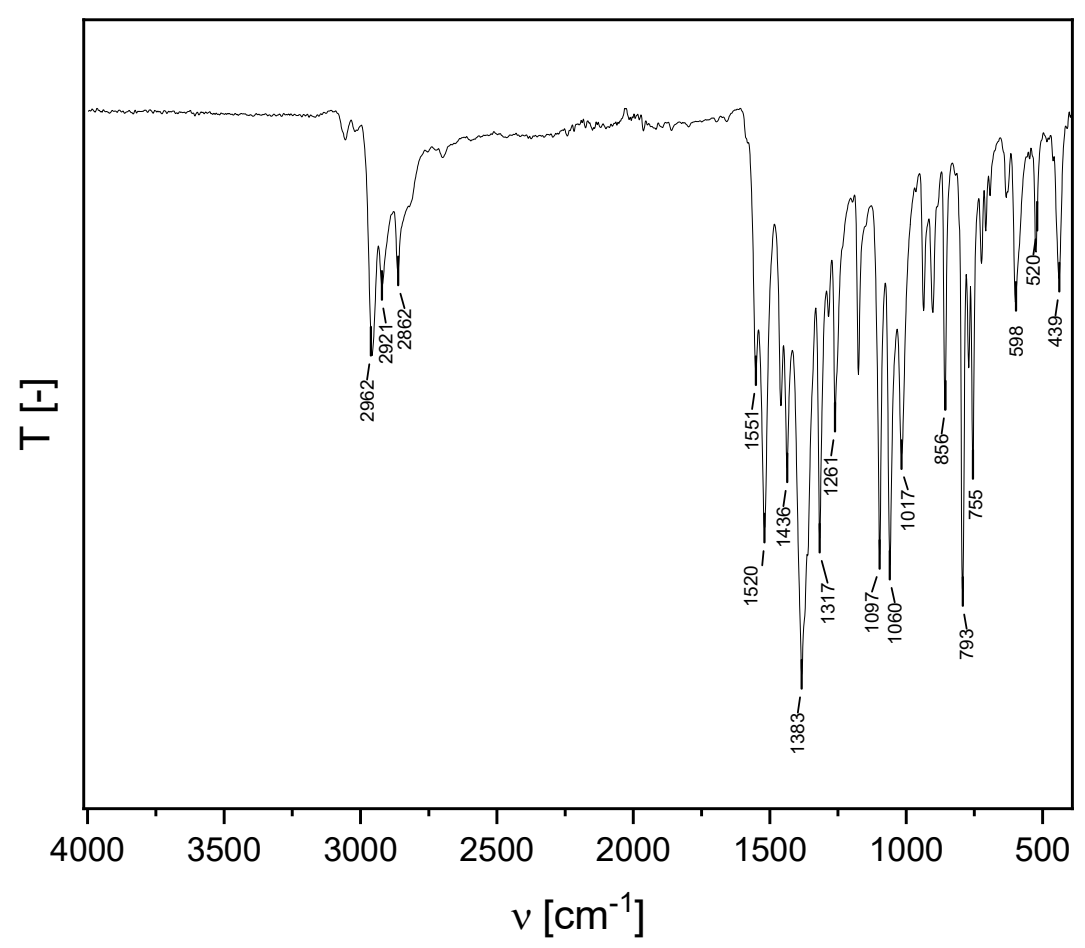

Fig. S30. ATR-IR spectrum of $\left[\{\mathrm{L}(\mathrm{EtO}) \mathrm{Ga}\}_{2}-\mu, \eta^{1: 1}-\mathrm{Bi}_{4}\right] 15$.

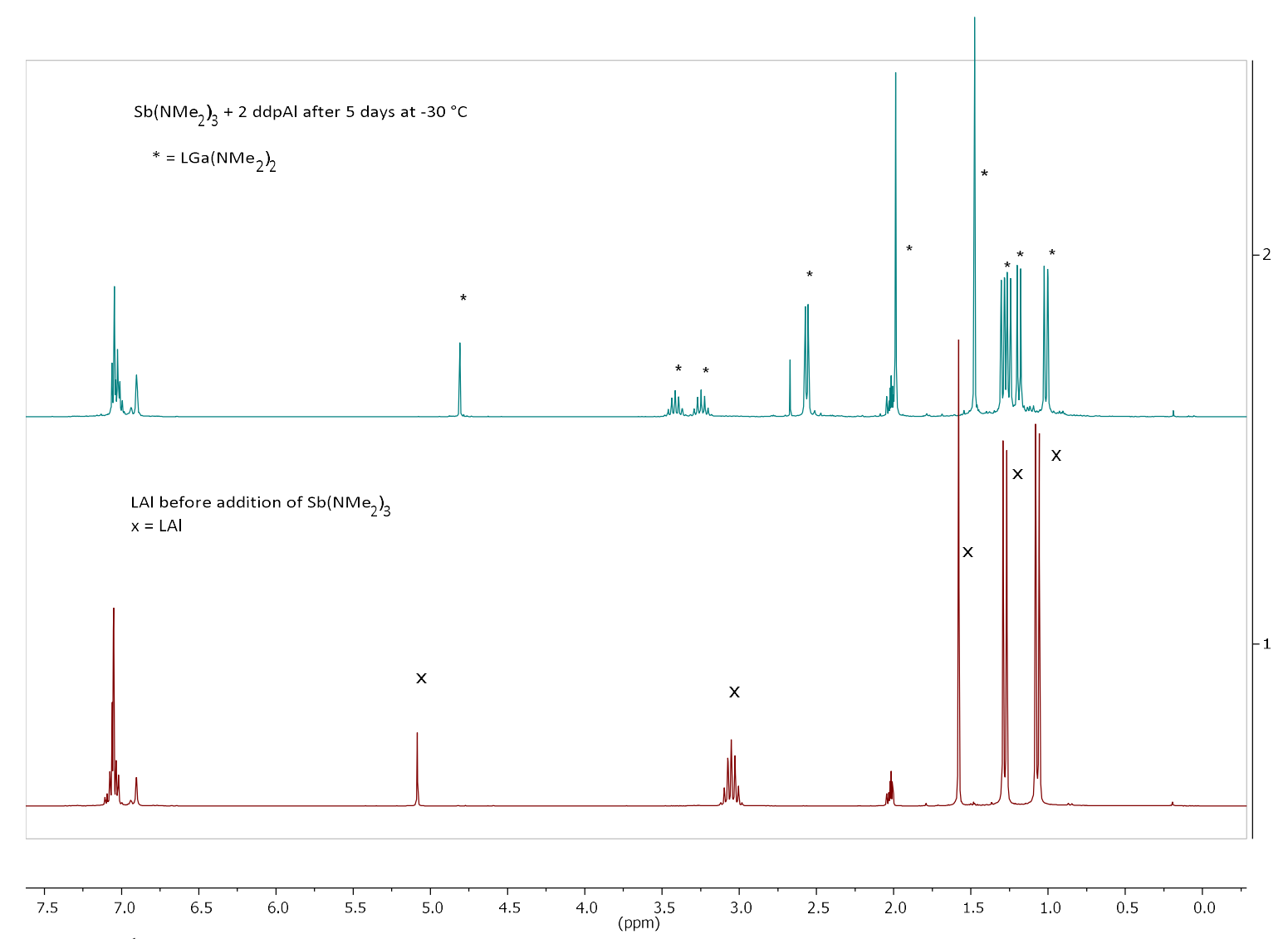

Fig. S31. ${ }^{1} \mathrm{H}$ NMR spectra of the reaction of $\mathrm{LAl}$ and $\mathrm{Sb}\left(\mathrm{NMe}_{2}\right)_{3}$ at $-30{ }^{\circ} \mathrm{C}$ in $\mathrm{C}_{7} \mathrm{D}_{8}$. 


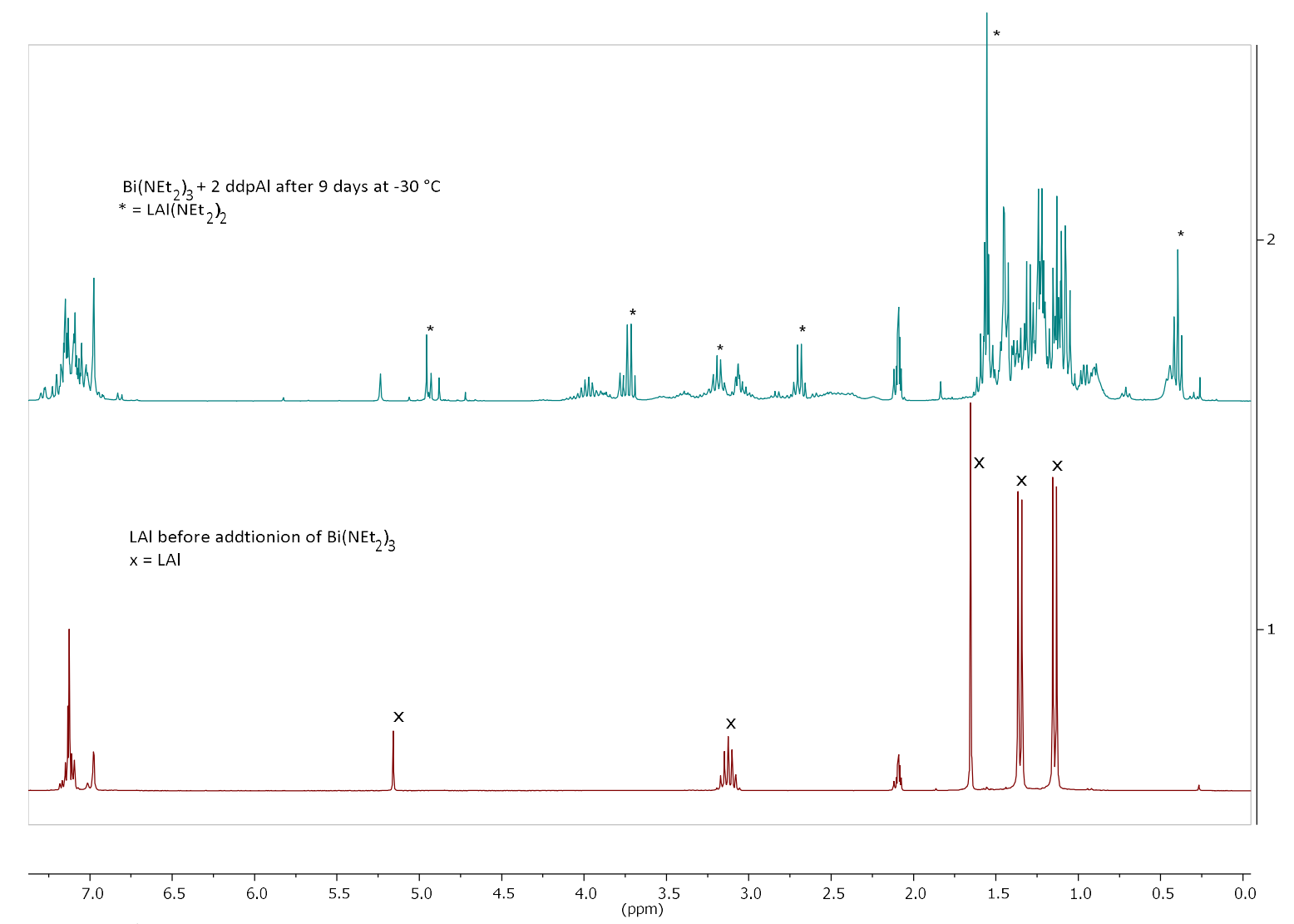

Fig. S32. ${ }^{1} \mathrm{H}$ NMR spectra of the reaction of $\mathrm{LAl}$ and $\mathrm{Bi}\left(\mathrm{NEt}_{2}\right)_{3}$ at $-30{ }^{\circ} \mathrm{C}$ in $\mathrm{C}_{7} \mathrm{D}_{8}$.

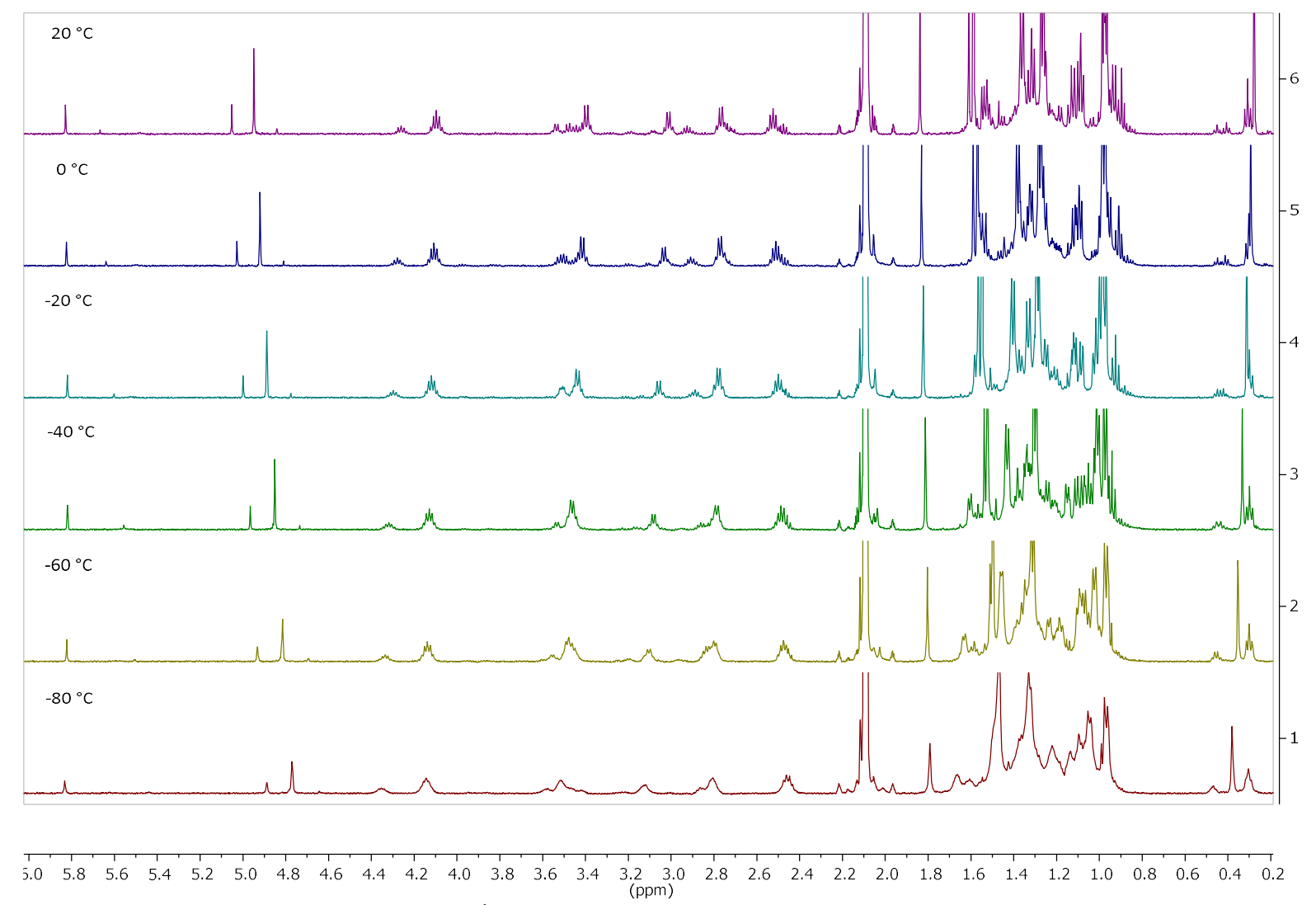

Fig. S33. Temperature dependent ${ }^{1} \mathrm{H}$ NMR spectra $\left(500 \mathrm{MHz}, \mathrm{C}_{6} \mathrm{D}_{6}\right)$ of $\left[\mathrm{L}\left(\mathrm{Et}_{2} \mathrm{~N}\right) \mathrm{AlBi}\right]_{2} 9$. 

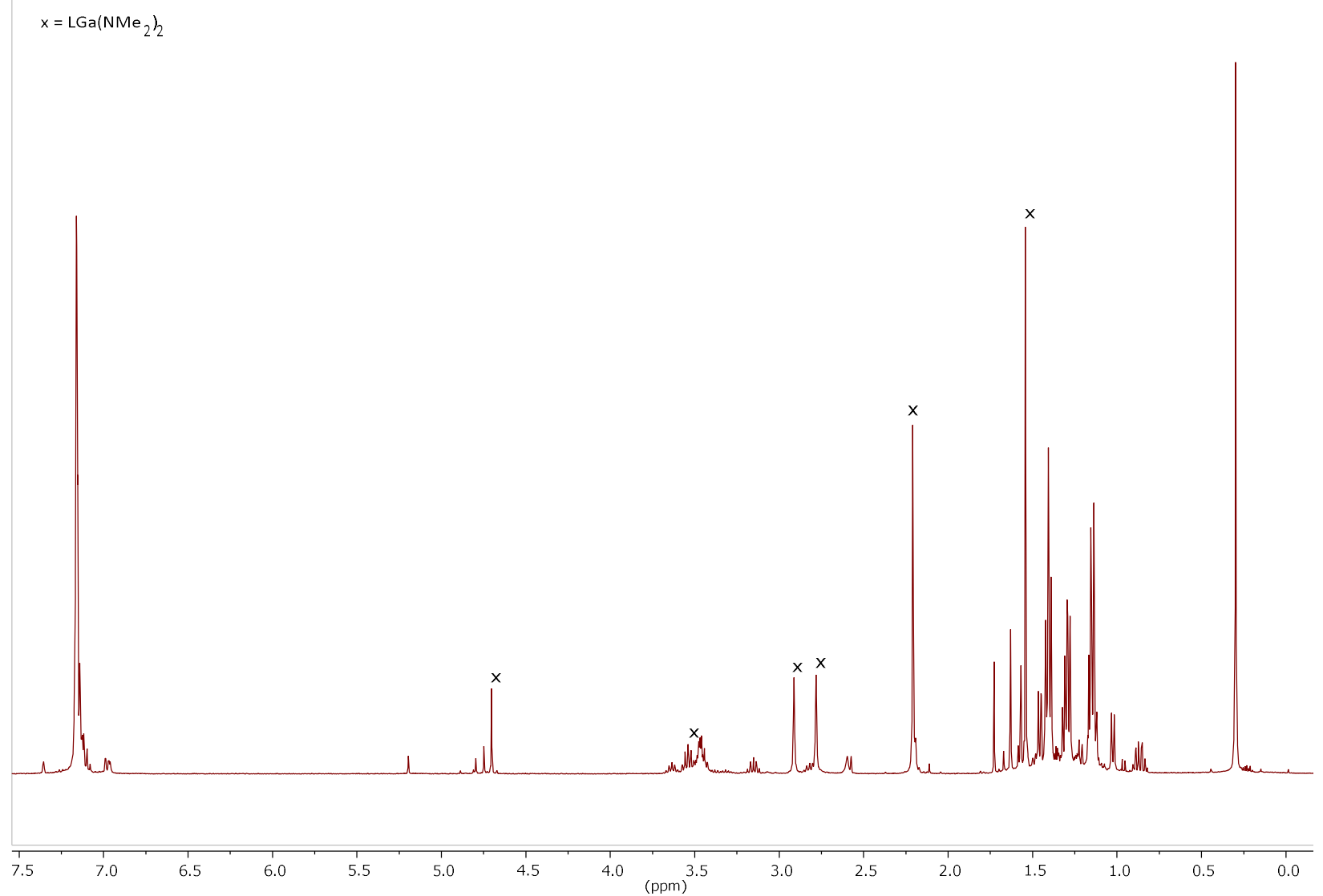

Fig. S34. ${ }^{1} \mathrm{H}$ NMR spectrum $\left(300 \mathrm{MHz}, \mathrm{C}_{6} \mathrm{D}_{6}, 25{ }^{\circ} \mathrm{C}\right)$ of the filtrate from the synthesis of $\left[\mathrm{L}\left(\mathrm{Me}_{2} \mathrm{~N}\right) \mathrm{GaBi}\right]_{2} \mathbf{1 0}$.

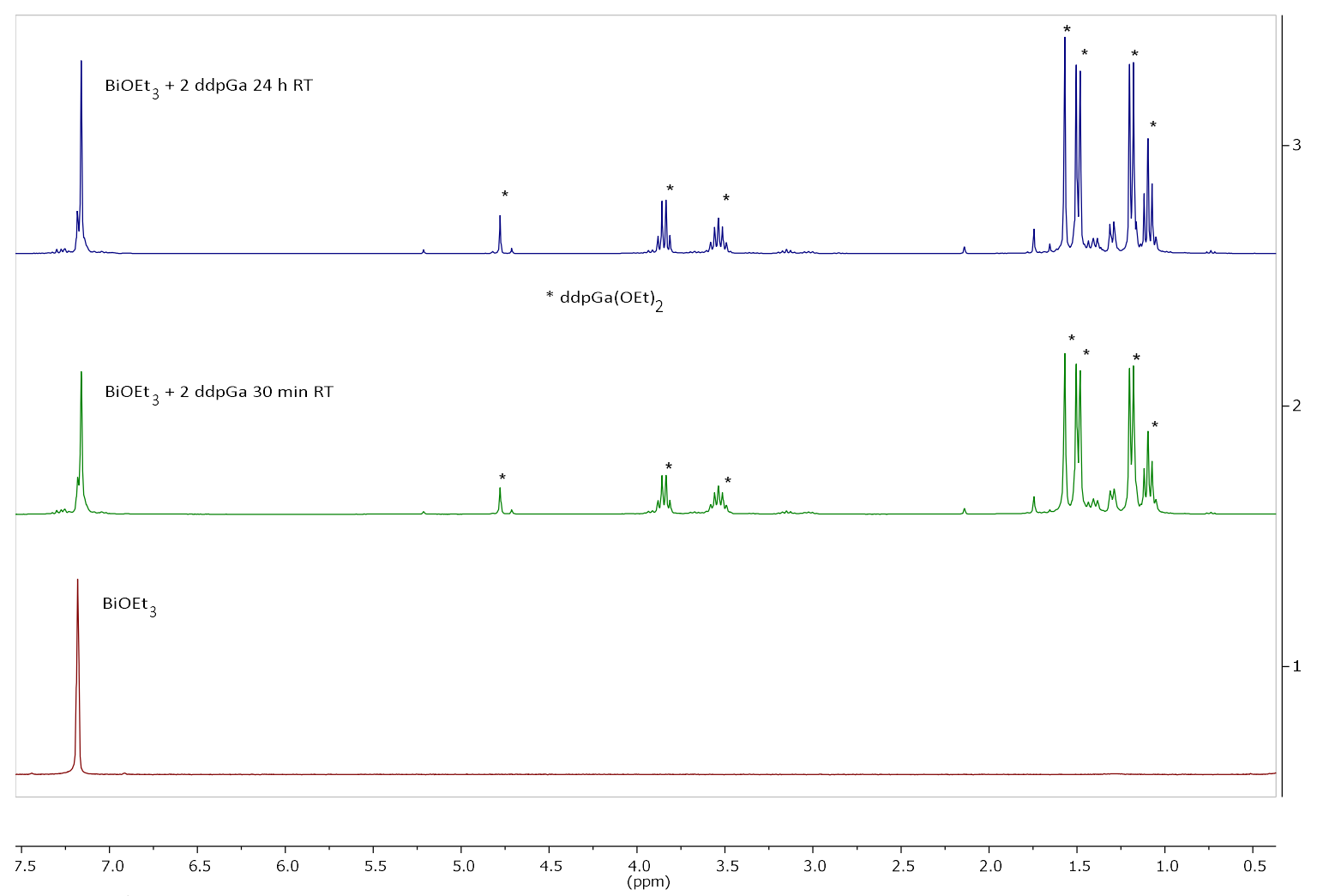

Fig. S35. ${ }^{1} \mathrm{H}$ NMR spectra $\left(300 \mathrm{MHz}, \mathrm{C}_{6} \mathrm{D}_{6}, 25^{\circ} \mathrm{C}\right)$ of the reaction of $\mathrm{LGa}$ and $\mathrm{Bi}(\mathrm{OEt})_{3}$. 


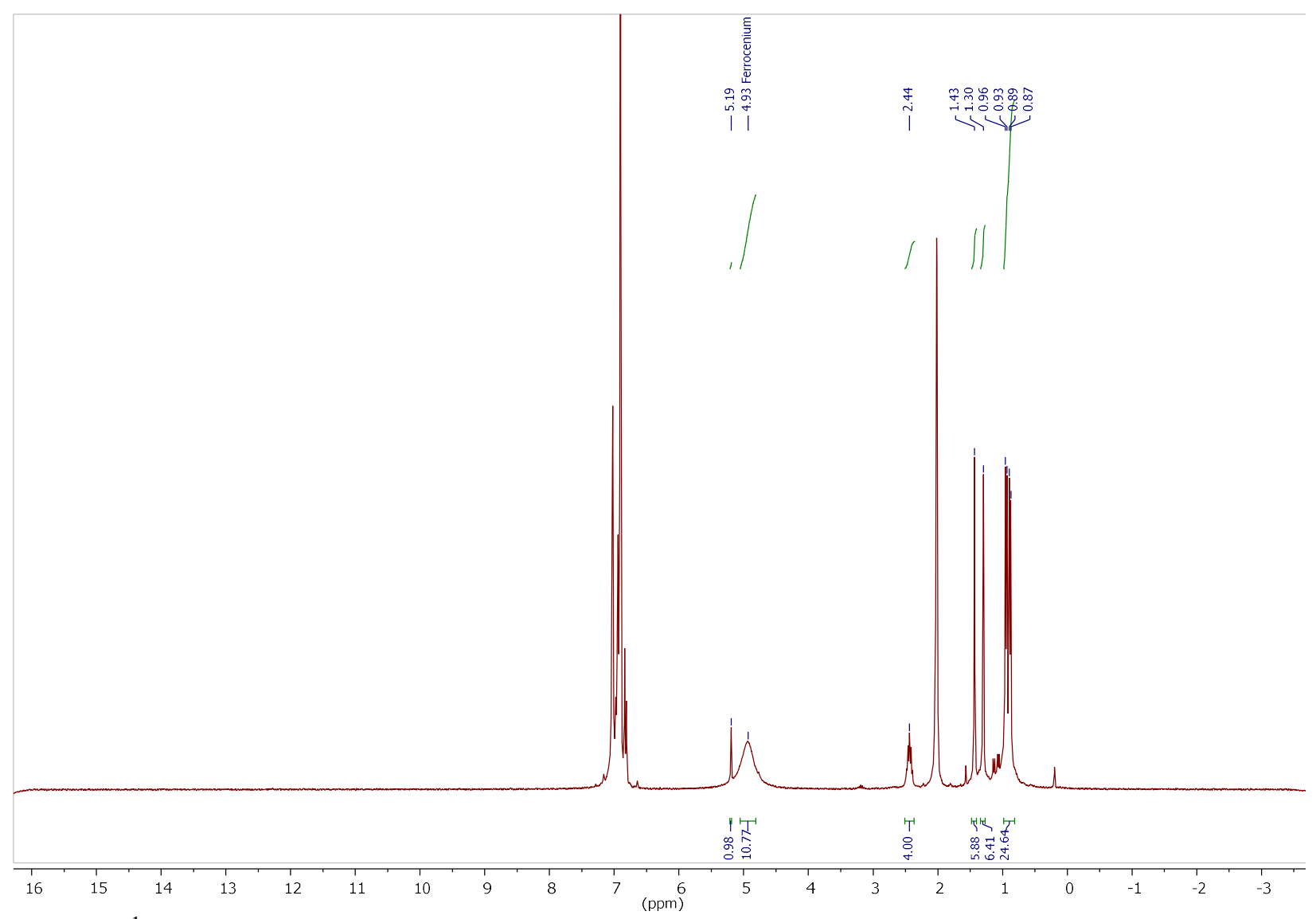

Fig. S36. ${ }^{1} \mathrm{H}$ NMR spectrum $\left(300 \mathrm{MHz}, \mathrm{C}_{7} \mathrm{D}_{8}, 25^{\circ} \mathrm{C}\right)$ of the reaction of $\left[\mathrm{L}\left(\mathrm{Me}_{2} \mathrm{~N}\right) \mathrm{AlSb}\right]_{2} 8$ and 2 eq. of $\mathrm{Fc}\left[\mathrm{B}\left(\mathrm{C}_{6} \mathrm{~F}_{5}\right)_{4}\right]$.

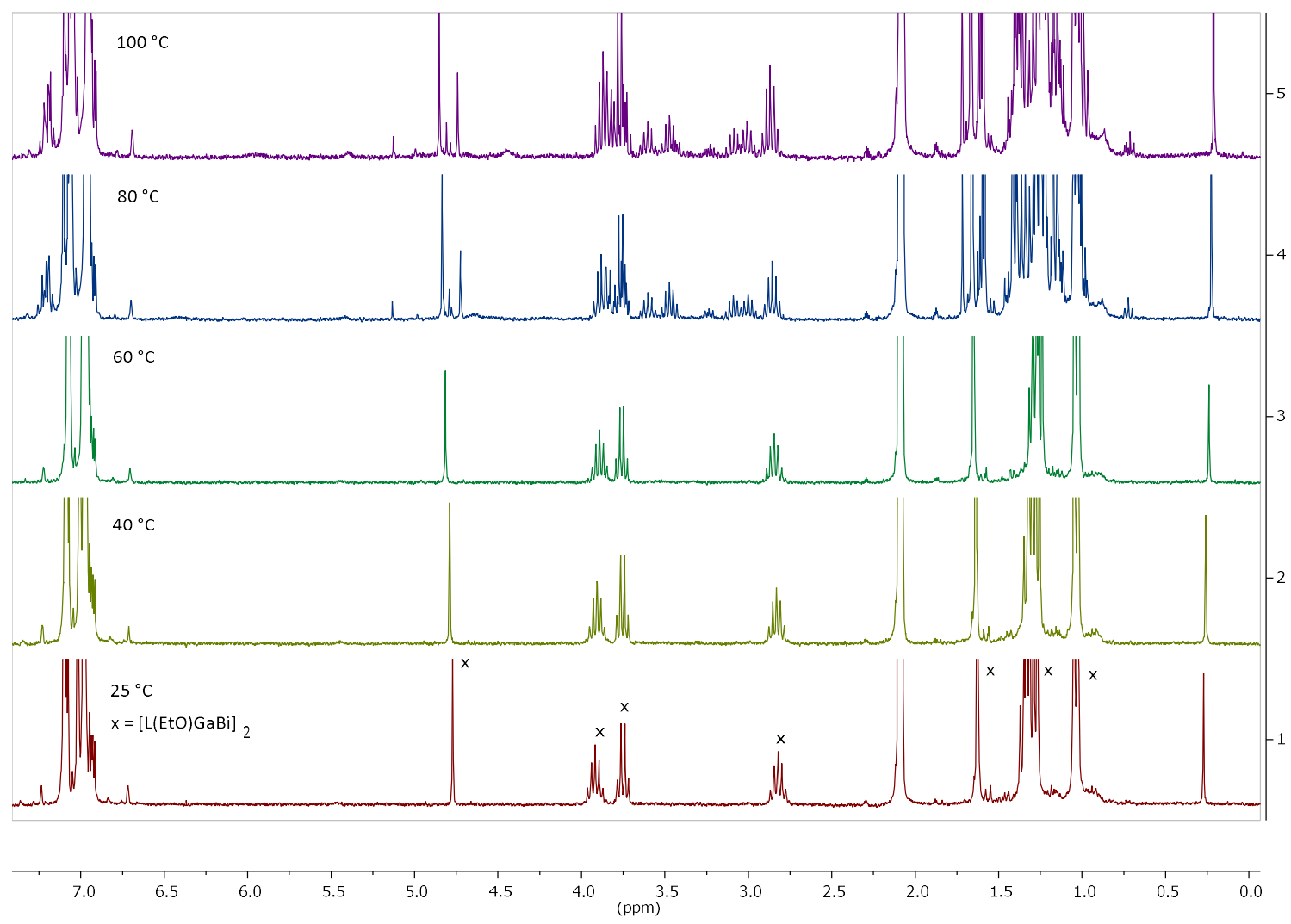

Fig. S37. Temperature dependent ${ }^{1} \mathrm{H}$ NMR spectra $\left(300 \mathrm{MHz}, \mathrm{C}_{7} \mathrm{D}_{8}\right)$ of $[\mathrm{L}(\mathrm{EtO}) \mathrm{GaBi}]_{2} 11$. 

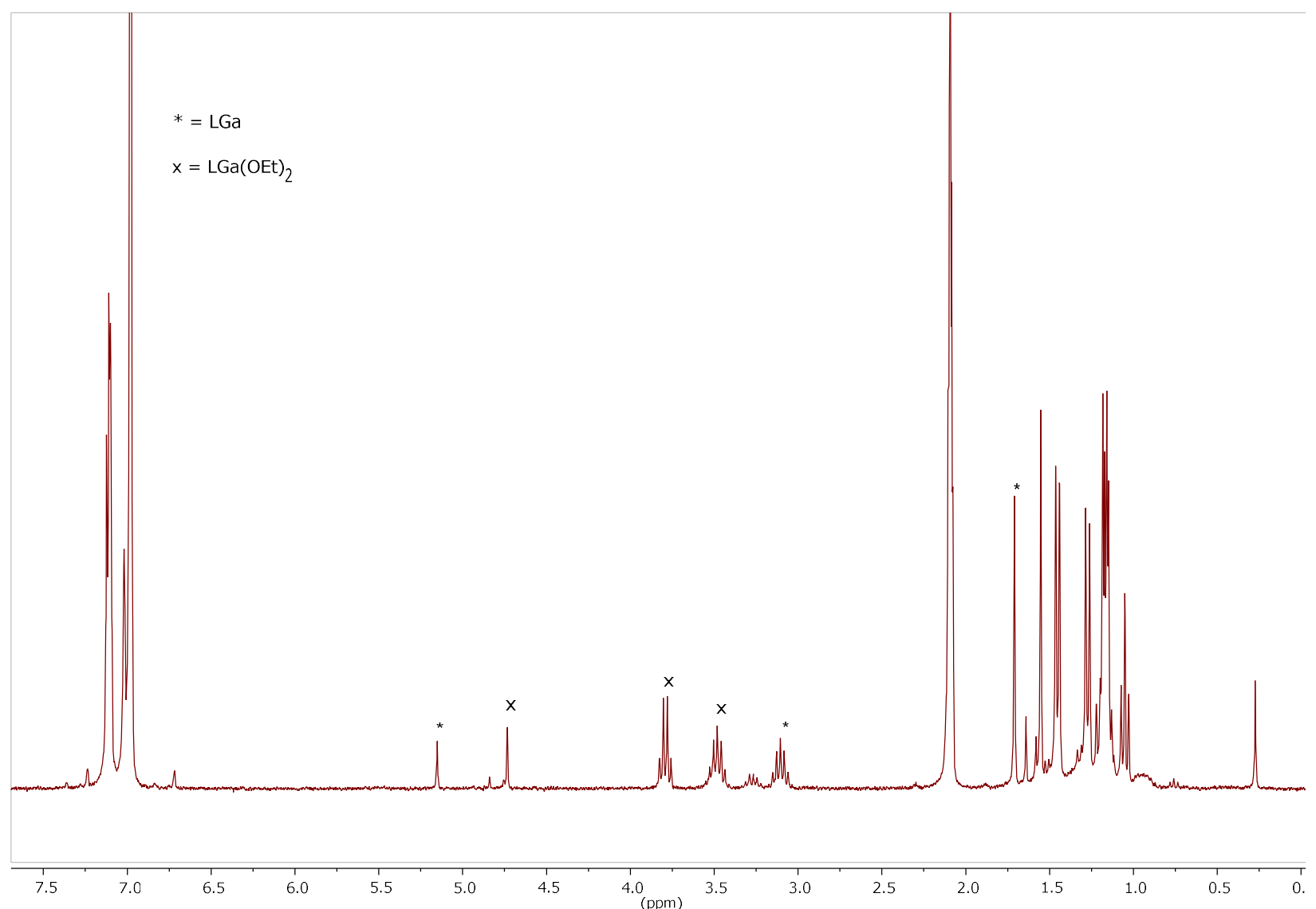

Fig. S38. ${ }^{1} \mathrm{H}$ NMR spectrum $\left(300 \mathrm{MHz}, \mathrm{C}_{7} \mathrm{D}_{8}, 25^{\circ} \mathrm{C}\right)$ after thermal treatment $(24 \mathrm{~h})$ of a solution of $[\mathrm{L}(\mathrm{EtO}) \mathrm{GaBi}]_{2} \mathbf{1 1}$ at $100{ }^{\circ} \mathrm{C}$. 


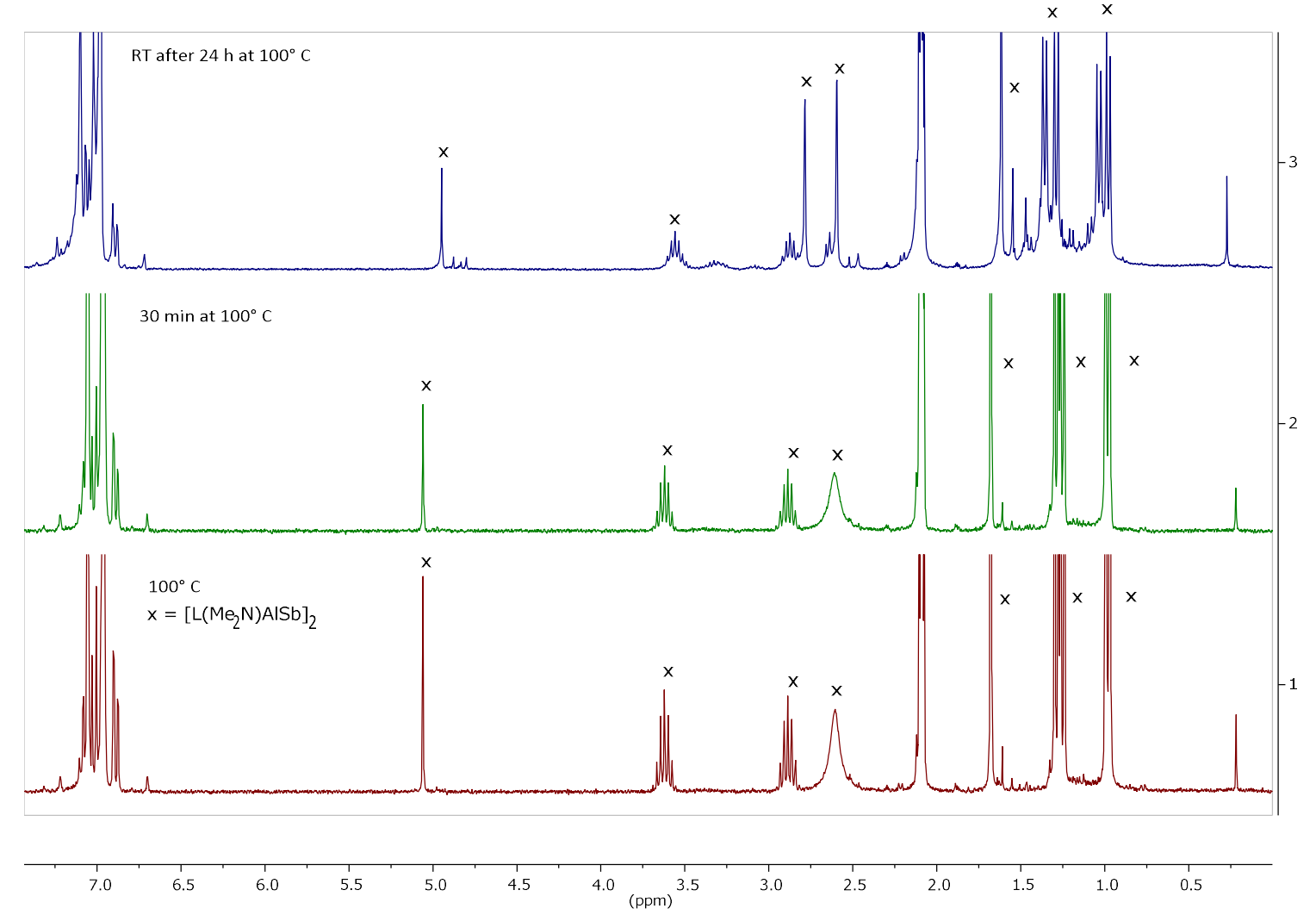

Fig. S39. ${ }^{1} \mathrm{H}$ NMR spectra $\left(300 \mathrm{MHz}, \mathrm{C}_{7} \mathrm{D}_{8}, 25^{\circ} \mathrm{C}\right)$ of $\left[\mathrm{L}\left(\mathrm{Me}_{2} \mathrm{~N}\right) \mathrm{AlSb}\right]_{2} 8$ after heating to $100{ }^{\circ} \mathrm{C}$ for $1 \mathrm{~min}, 30 \mathrm{~min}$ and $24 \mathrm{~h}$ in a aluminum heating block.
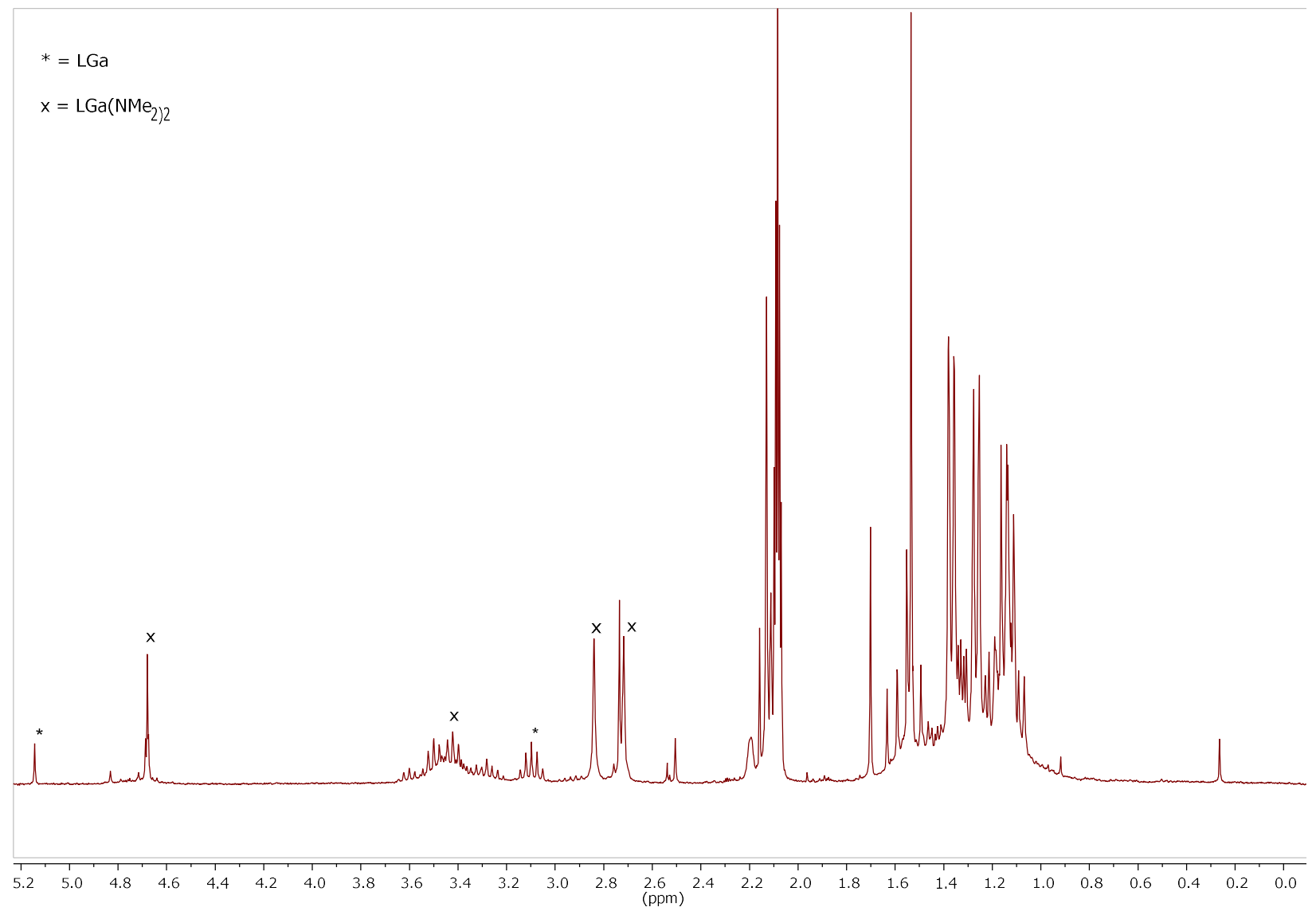

Fig. S40. ${ }^{1} \mathrm{H}$ NMR spectrum $\left(300 \mathrm{MHz}, \mathrm{C}_{7} \mathrm{D}_{8}, 25^{\circ} \mathrm{C}\right)$ after thermal treatment $(24 \mathrm{~h})$ of a solution of $\left[\mathrm{L}\left(\mathrm{Me}_{2} \mathrm{~N}\right) \mathrm{GaBi}\right]_{2} \mathbf{1 0}$ at $100{ }^{\circ} \mathrm{C}$. 


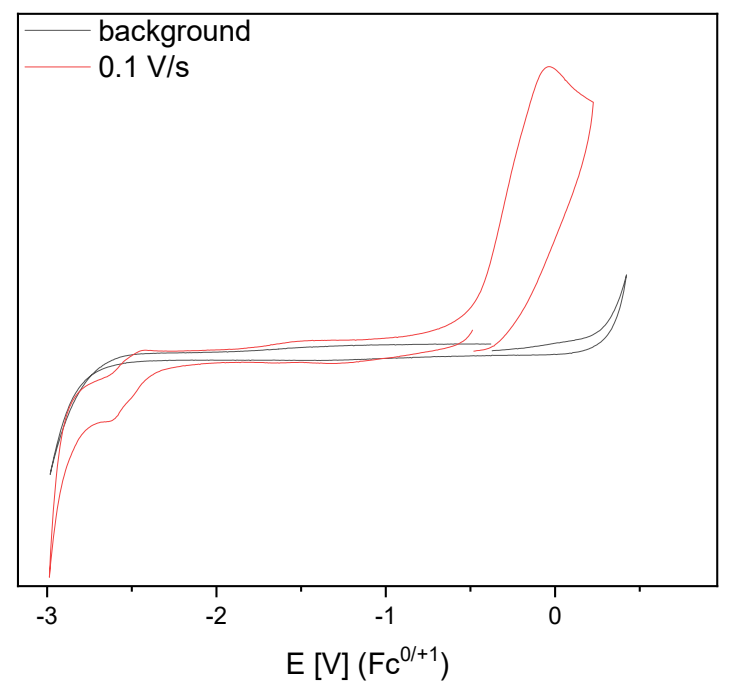

Fig. S41. Cyclic voltammograms of $\left[\mathrm{L}\left(\mathrm{Me}_{2} \mathrm{~N}\right) A 1 A s\right]_{2} 1$ in $\mathrm{THF}$ solution $(1 \mathrm{mM})$ at ambient temperature containing $\left[n-\mathrm{Bu}_{4} \mathrm{~N}\right]\left[\mathrm{PF}_{6}\right](100 \mathrm{mM})$ as electrolyte.

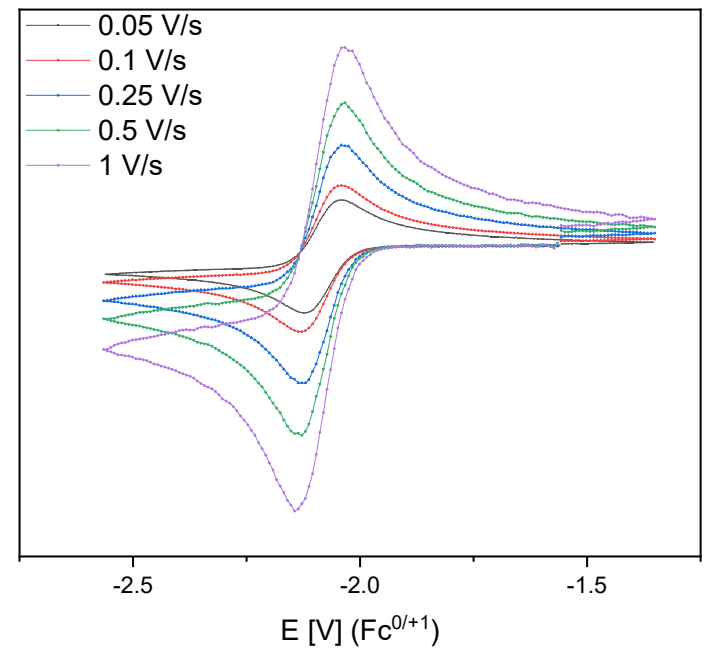

Fig. S42. Cyclic voltammograms of $[\mathrm{L}(\mathrm{EtO}) \mathrm{GaAs}]_{2} \mathbf{2}$ in THF solution $(1 \mathrm{mM})$ at ambient temperature containing $\left[n-\mathrm{Bu}_{4} \mathrm{~N}\right]\left[\mathrm{PF}_{6}\right](100 \mathrm{mM})$ as electrolyte at varying scan rate showing the reversible reduction event.

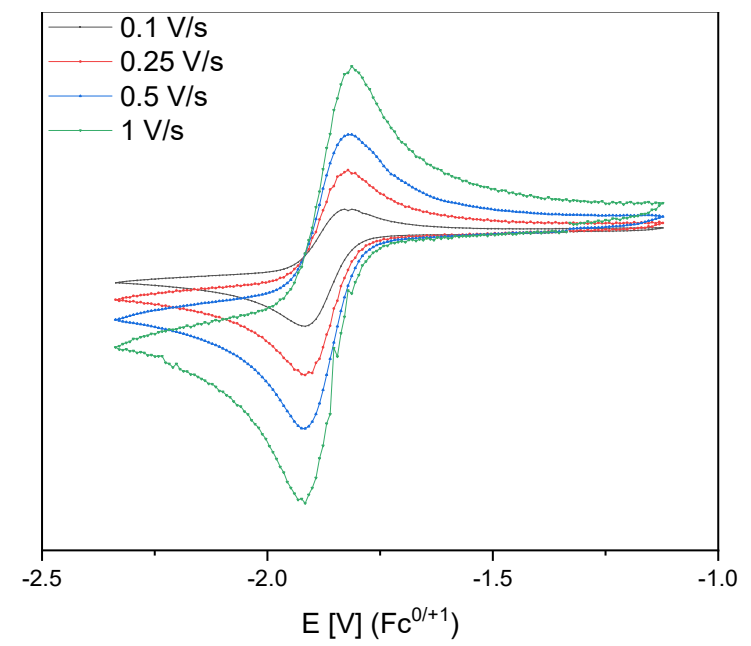

Fig. S43. Cyclic voltammograms of $[\mathrm{L}(\mathrm{Cl}) \mathrm{GaAs}]_{2} 3$ in THF solution $(1 \mathrm{mM})$ at ambient temperature containing $\left[n-\mathrm{Bu}_{4} \mathrm{~N}\right]\left[\mathrm{PF}_{6}\right](100 \mathrm{mM})$ as electrolyte at varying scan rate showing the reversible reduction event. 


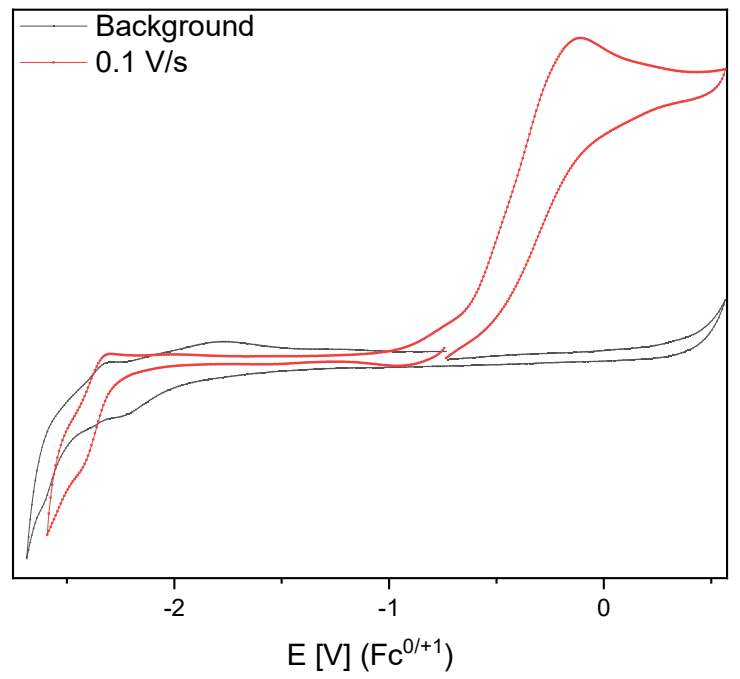

Fig. S44. Cyclic voltammograms of $\left[\mathrm{L}\left(\mathrm{Me}_{2} \mathrm{~N}\right) \mathrm{AlSb}\right]_{2} 8$ in $\mathrm{THF}$ solution $(1 \mathrm{mM})$ at ambient temperature containing $\left[n-\mathrm{Bu}_{4} \mathrm{~N}\right]\left[\mathrm{BARF}^{24}\right](100 \mathrm{mM})$ as electrolyte.

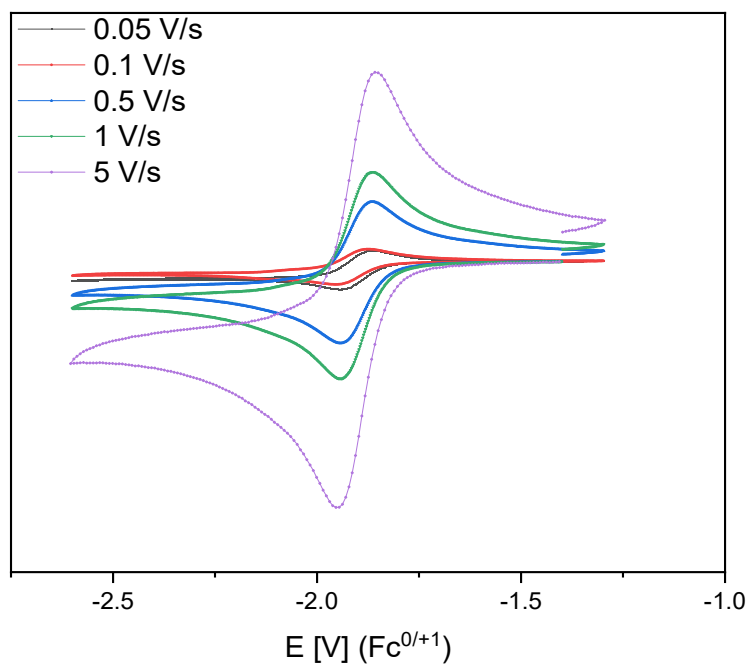

Fig. S45. Cyclic voltammograms of $\left[\mathrm{L}\left(\mathrm{Me}_{2} \mathrm{~N}\right) \mathrm{GaSb}_{2} 4\right.$ in THF solution $(1 \mathrm{mM})$ at $45{ }^{\circ} \mathrm{C}$ containing $\left[n-\mathrm{Bu}_{4} \mathrm{~N}\right]\left[\mathrm{PF}_{6}\right](100 \mathrm{mM})$ as electrolyte at varying scan rate showing the reversible reduction event. 


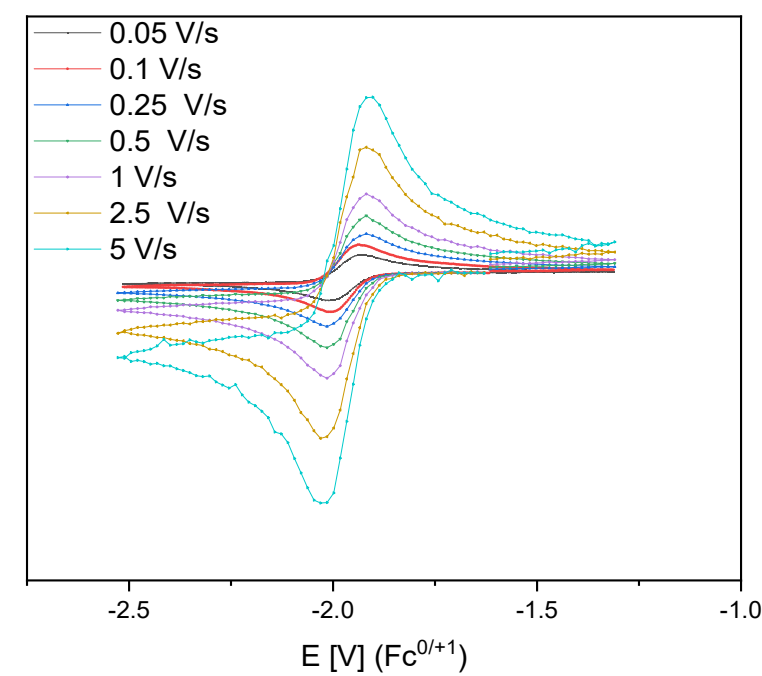

Fig. S46. Cyclic voltammograms of $[\mathrm{L}(\mathrm{EtO}) \mathrm{GaSb}]_{2} 5$ in THF solution $(1 \mathrm{mM})$ at ambient temperature containing $\left[n-\mathrm{Bu}_{4} \mathrm{~N}\right]\left[\mathrm{PF}_{6}\right](100 \mathrm{mM})$ as electrolyte at varying scan rate showing the reversible reduction event.

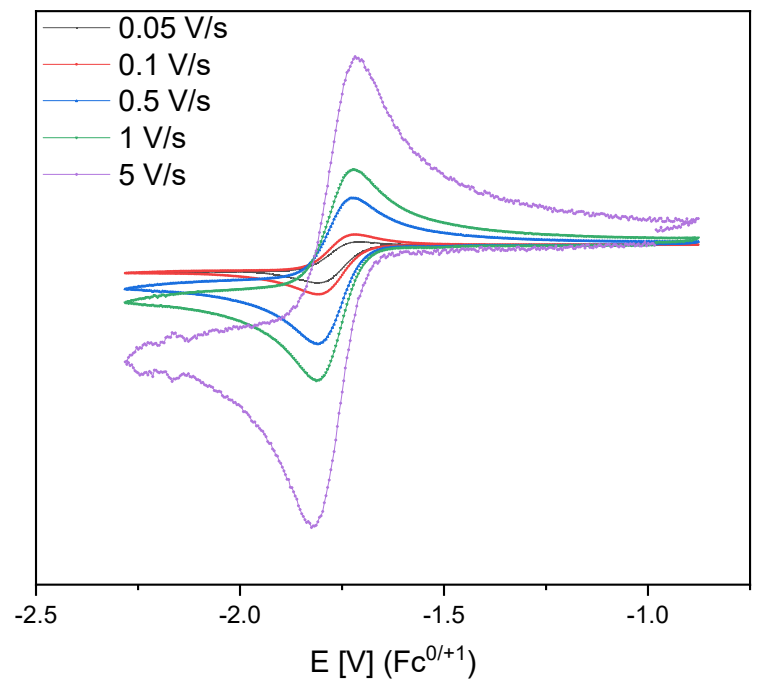

Fig. S47. Cyclic voltammograms of $[\mathrm{L}(\mathrm{Cl}) \mathrm{GaSb}]_{2} 6$ in THF solution $(1 \mathrm{mM})$ at $45{ }^{\circ} \mathrm{C}$ containing $[n-$ $\left.\mathrm{Bu}_{4} \mathrm{~N}\right]\left[\mathrm{PF}_{6}\right](100 \mathrm{mM})$ as electrolyte at varying scan rate showing the reversible reduction event. 


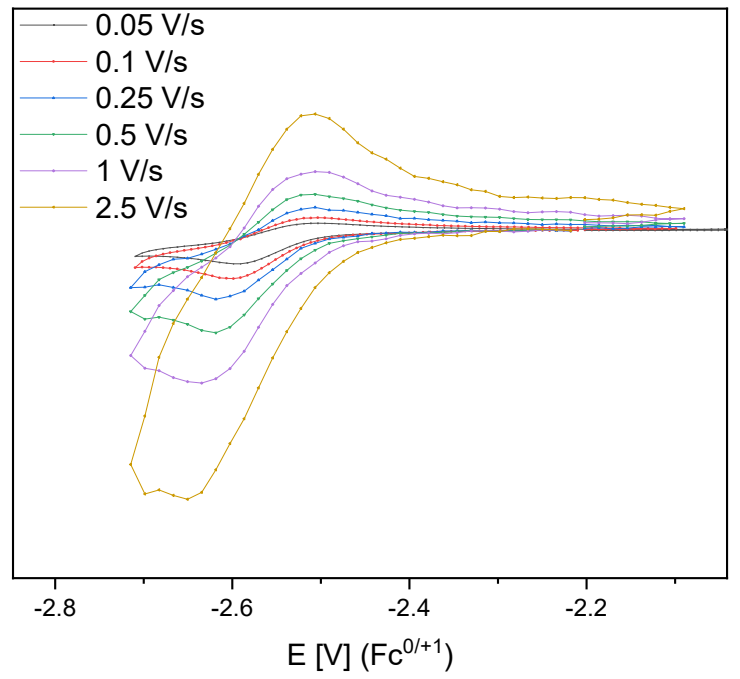

Fig. S48. Cyclic voltammograms of $\left[\mathrm{L}\left(\mathrm{Et}_{2} \mathrm{~N}\right) \mathrm{AlBi}\right]_{2} 9$ in THF solution $(1 \mathrm{mM})$ at ambient temperature containing $\left[n-\mathrm{Bu}_{4} \mathrm{~N}\right]\left[\mathrm{PF}_{6}\right](100 \mathrm{mM})$ as electrolyte at varying scan rate showing s pseudo reversible reduction event.

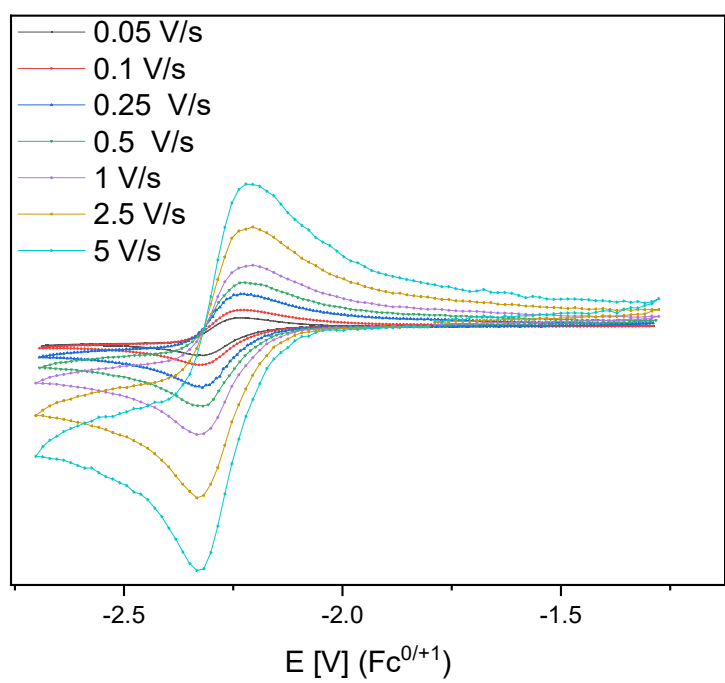

Fig. S49. Cyclic voltammograms of $\left[\mathrm{L}\left(\mathrm{Me}_{2} \mathrm{~N}\right) \mathrm{GaBi}\right]_{2} 10$ in $\mathrm{THF}$ solution $(1 \mathrm{mM})$ at $45{ }^{\circ} \mathrm{C}$ containing $\left[n-\mathrm{Bu}_{4} \mathrm{~N}\right]\left[\mathrm{PF}_{6}\right](100 \mathrm{mM})$ as electrolyte at varying scan rate showing the reversible reduction event. 


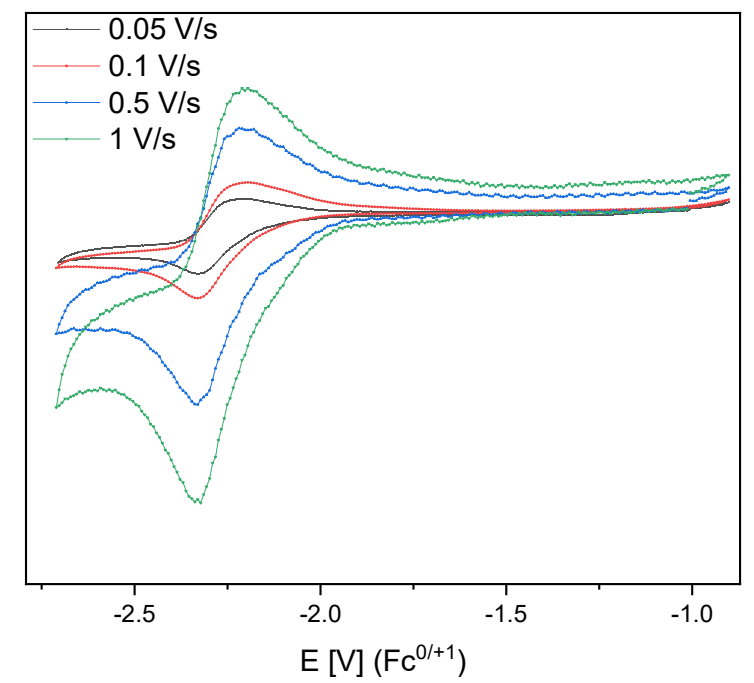

Fig. S50. Cyclic voltammograms of $\left[\mathrm{L}\left(\mathrm{Et}_{2} \mathrm{~N}\right) \mathrm{GaBi}\right]_{2} 7$ in THF solution $(1 \mathrm{mM})$ at $45{ }^{\circ} \mathrm{C}$ containing $\left[n-\mathrm{Bu}_{4} \mathrm{~N}\right]\left[\mathrm{PF}_{6}\right](100 \mathrm{mM})$ as electrolyte at varying scan rate showing the reversible reduction event.

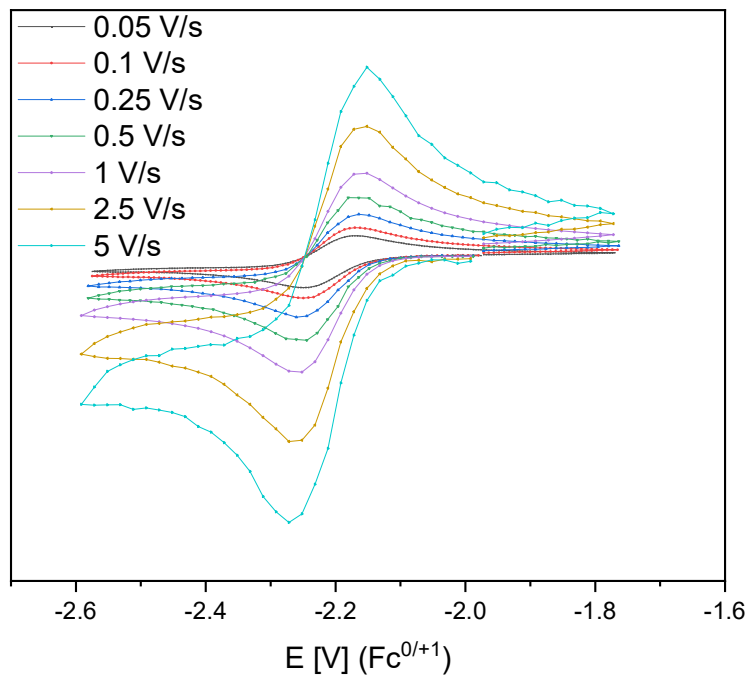

Fig. S51. Cyclic voltammograms of $[\mathrm{L}(\mathrm{EtO}) \mathrm{GaBi}]_{2} 11$ in $\mathrm{THF}$ solution $(1 \mathrm{mM})$ at ambient temperature containing $\left[n-\mathrm{Bu}_{4} \mathrm{~N}\right]\left[\mathrm{PF}_{6}\right](100 \mathrm{mM})$ as electrolyte at varying scan rate showing the reversible reduction event. 


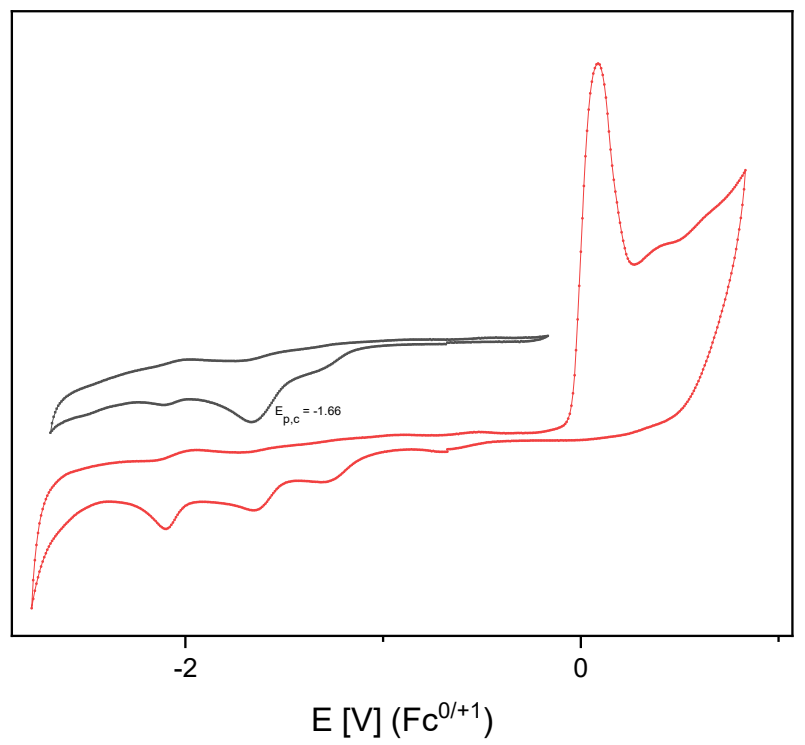

Fig. S52. Cyclic voltammograms of $[\mathrm{L}(\mathrm{TfO}) \mathrm{GaBi}]_{2}$ in THF solution $(1 \mathrm{mM})$ at ambient temperature containing $\left[n-\mathrm{Bu}_{4} \mathrm{~N}\right][\mathrm{BARF}](50 \mathrm{mM})$ as electrolyte. In black first scan. The purple color degraded during the measurement indicating decomposition similar to the aluminum substituted dipnictenes. No sign of an reversible reduction event was observed even at fist cycle (circa $30 \mathrm{~s}$ after addition of $\left.[\mathrm{L}(\mathrm{TfO}) \mathrm{GaBi}]_{2}\right)$, but a irreversible reduction at $-1.66 \mathrm{~V}$ which faded during the measurements.

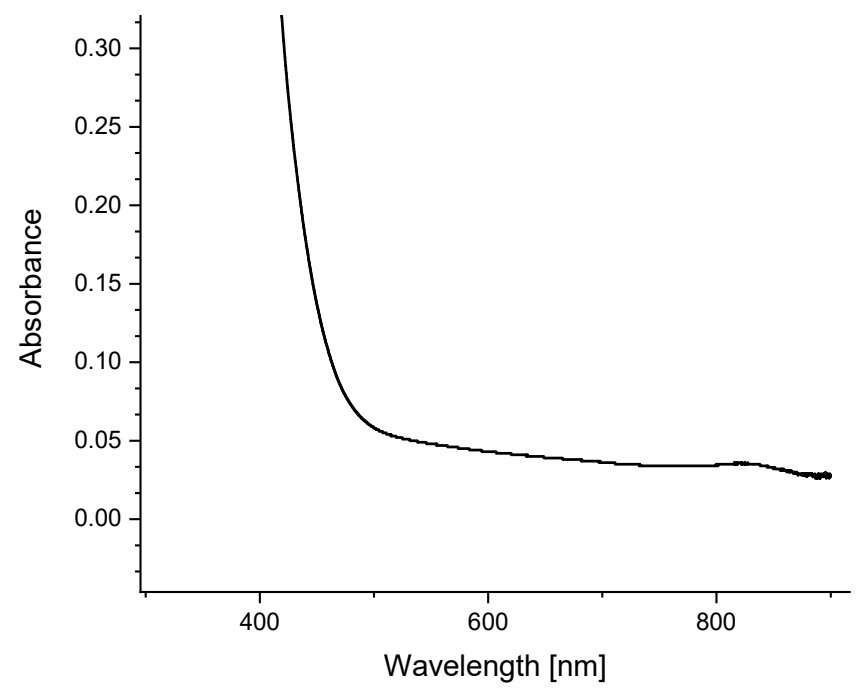

Fig. S53. UV-VIS spectrum of $\left[\mathrm{L}\left(\mathrm{Me}_{2} \mathrm{~N}\right) \mathrm{AlAs}\right]_{2} \mathbf{1}$ in benzene. 


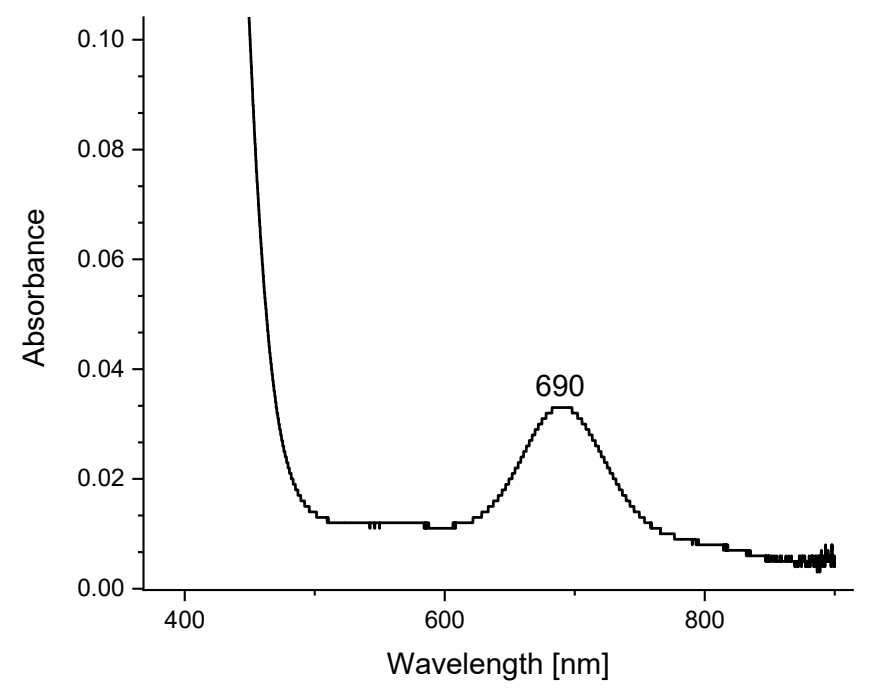

Fig. S54. UV-VIS spectrum of $[\mathrm{L}(\mathrm{EtO}) \mathrm{GaAs}]_{2} 2$ in benzene.

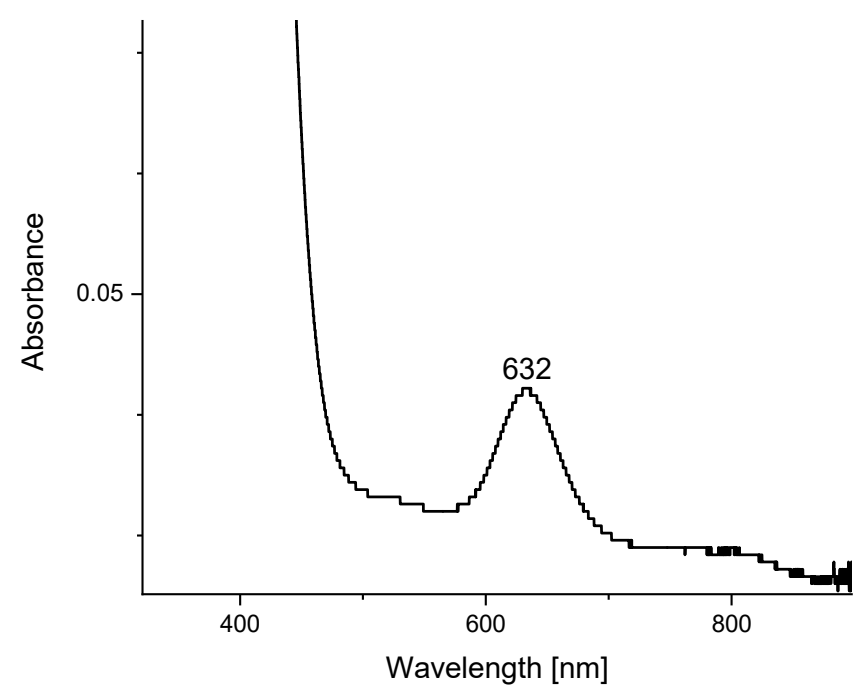

Fig. S55. UV-VIS spectrum of $[\mathrm{L}(\mathrm{Cl}) \mathrm{GaAs}]_{2} 3$ in benzene.

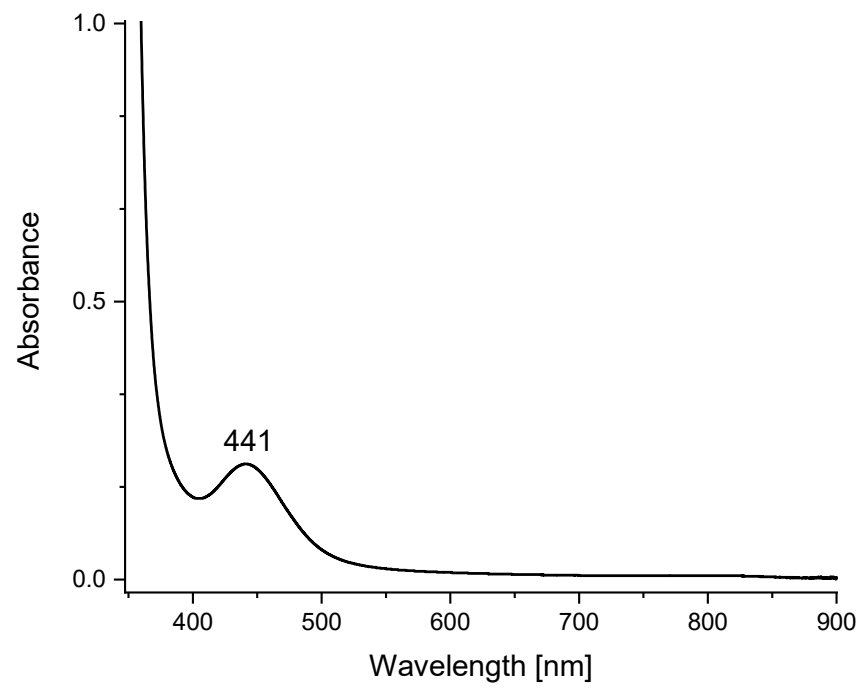

Fig. S56. UV-VIS spectrum of $\left[\mathrm{L}\left(\mathrm{Me}_{2} \mathrm{~N}\right) \mathrm{AlSb}\right]_{2} 8$ in benzene. Decomposed during measurement. 


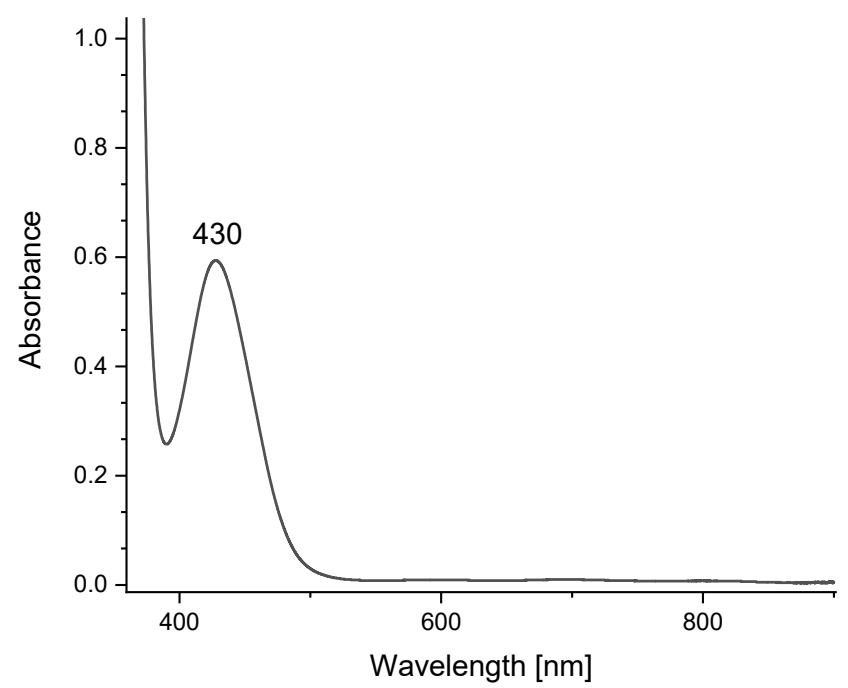

Fig. S57. UV-VIS spectrum of $\left[\mathrm{L}\left(\mathrm{Me}_{2} \mathrm{~N}\right) \mathrm{GaSb}\right]_{2} 4$ in benzene.

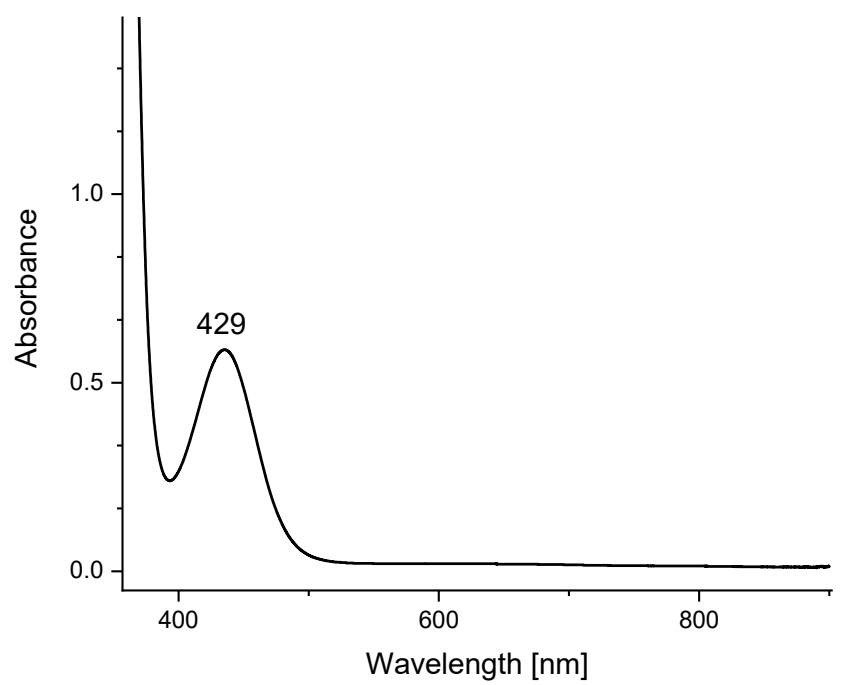

Fig. S58. UV-VIS spectrum of $\left[\mathrm{L}(\mathrm{EtO}) \mathrm{GaSb}_{2} \mathbf{5}\right.$ in benzene.

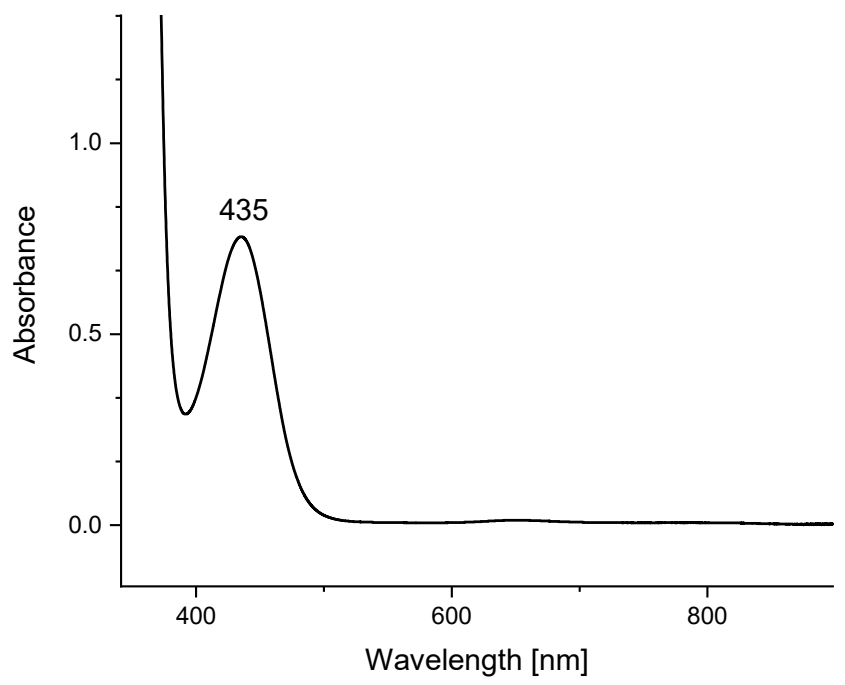

Fig. S59. UV-VIS spectrum of $[\mathrm{L}(\mathrm{Cl}) \mathrm{GaSb}]_{2} 6$ in benzene. 


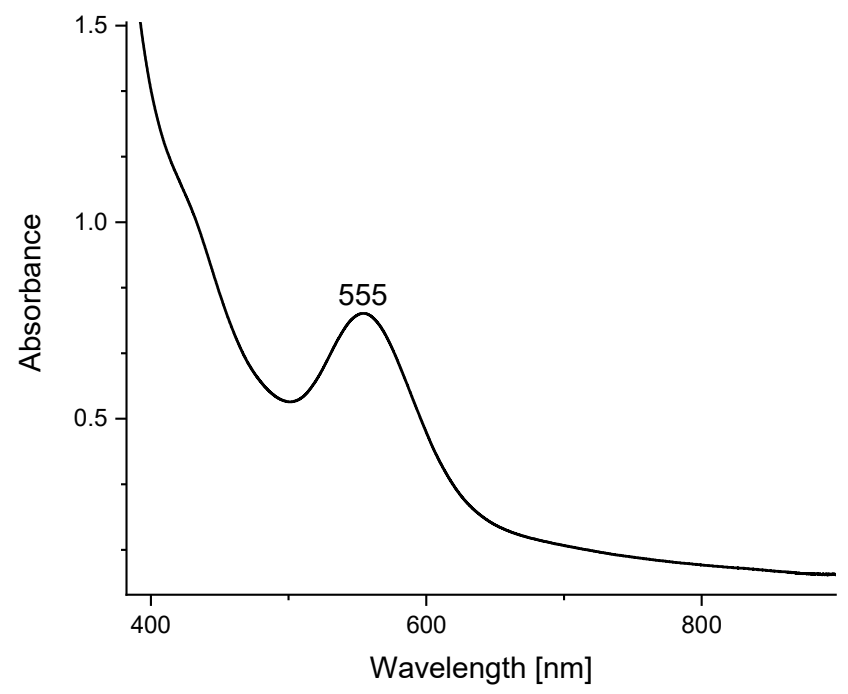

Fig. S60. UV-VIS spectrum of $\left[\mathrm{L}\left(\mathrm{Et}_{2} \mathrm{~N}\right) \mathrm{AlBi}_{2} \mathbf{9}\right.$ in benzene.

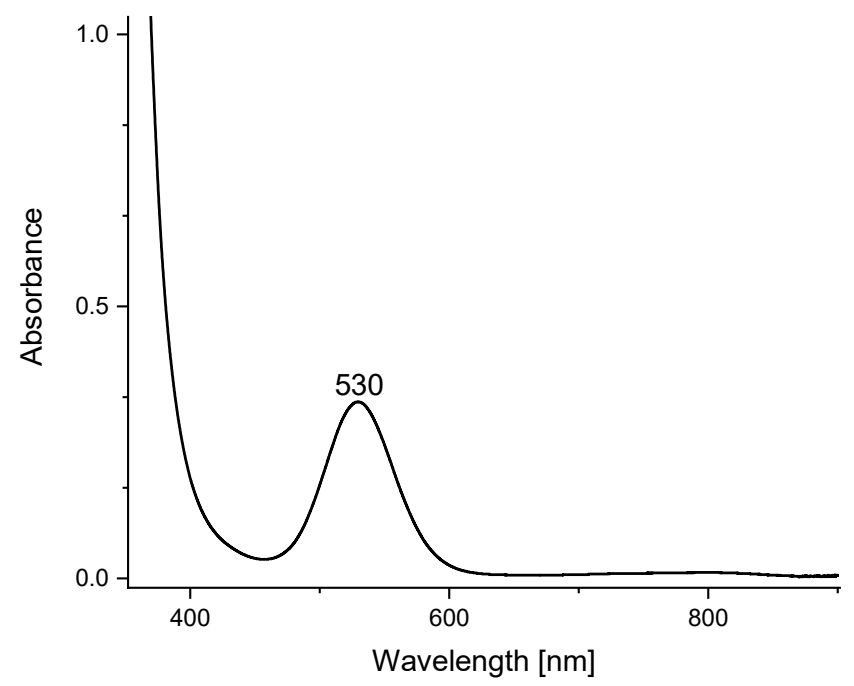

Fig. S61. UV-VIS spectrum of $\left[\mathrm{L}\left(\mathrm{Me}_{2} \mathrm{~N}\right) \mathrm{GaBi}\right]_{2} \mathbf{1 0}$ in benzene.

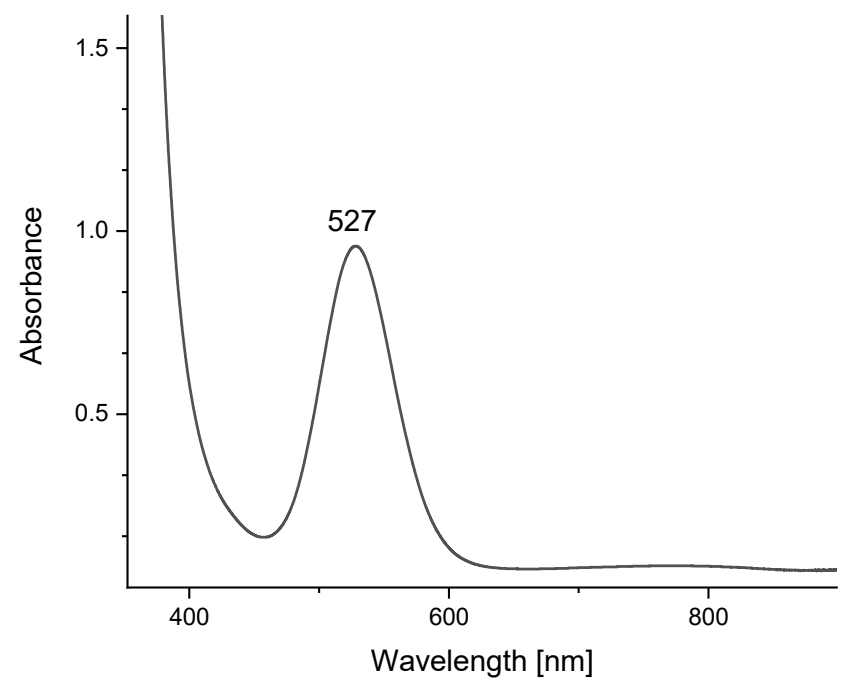

Fig. S62. UV-VIS spectrum of $\left[\mathrm{L}\left(\mathrm{Et}_{2} \mathrm{~N}\right) \mathrm{GaBi}\right]_{2} \mathbf{7}$ in benzene. 


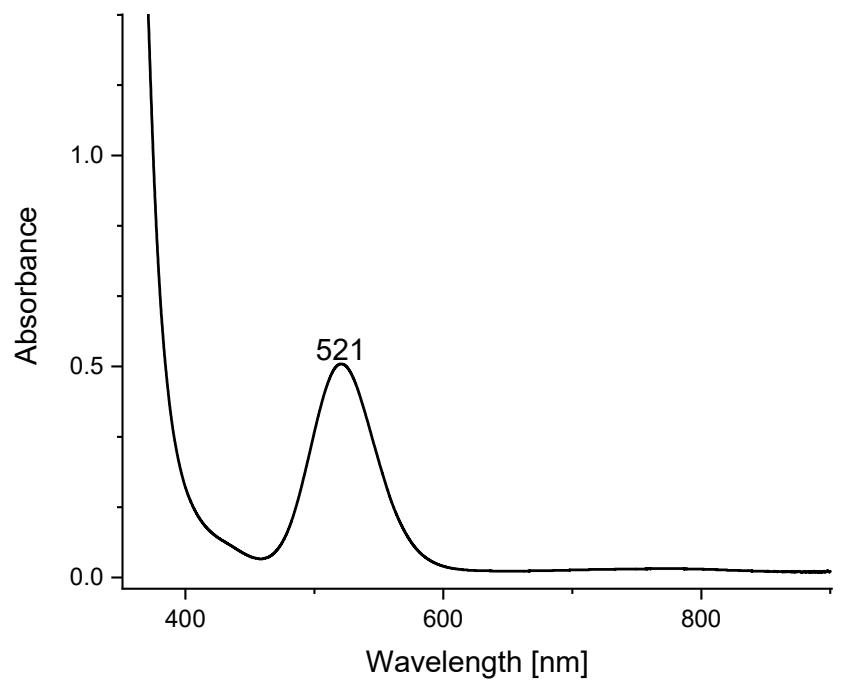

Fig. S63. UV-VIS spectrum of $[\mathrm{L}(\mathrm{EtO}) \mathrm{GaBi}]_{2} \mathbf{1 1}$ in benzene.

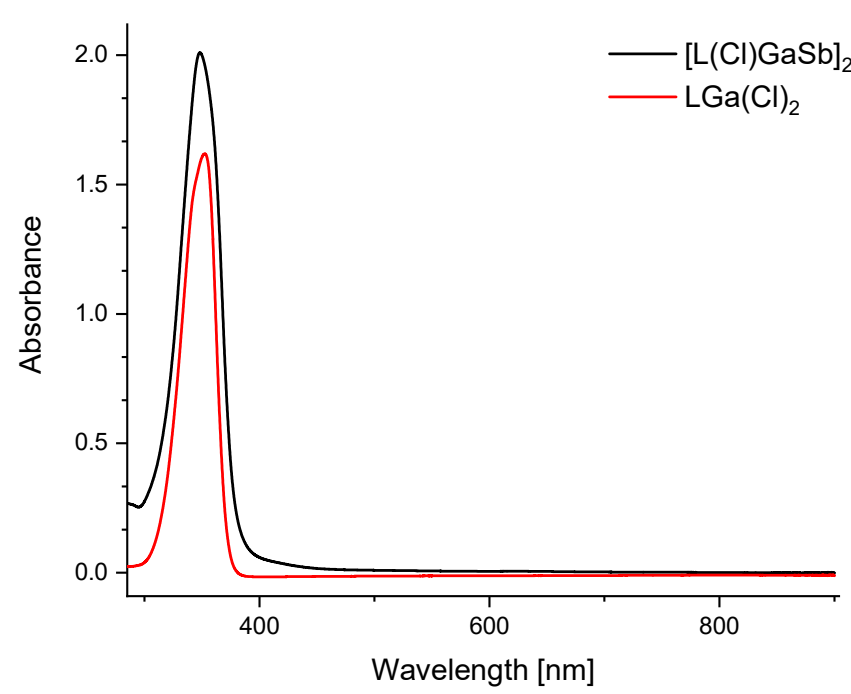

Fig. S64. UV-VIS spectra of $[\mathrm{L}(\mathrm{Cl}) \mathrm{GaAs}]_{2} \mathbf{3}$ and $\mathrm{LGa}(\mathrm{Cl})_{2}$. 


\section{Crystallographic Details.}

The crystals were mounted on nylon loops in inert oil. Data of 9, 11, and $\mathbf{1 5}$ were collected on a Bruker AXS D8 Kappa diffractometer with APEX2 detector (mono-chromated $\mathrm{Mo}_{K a}$ radiation, $l=$ $0.71073 \AA$ ) while the data of $\mathbf{8 , 1 0}$, and $\mathbf{1 3}$ were collected on a Bruker AXS D8 Venture diffractometer with Photon II detector (mono-chromated $\mathrm{Cu}_{K a}$ radiation, $l=1.54178 \AA$, micro-focus source) at 100(2) K. The structures were solved by Direct Methods (SHELXS-97) ${ }^{7}$ and refined anisotropically by full-matrix least-squares on $F^{2}$ (SHELXL-2014) ${ }^{8,9}$. Absorption corrections were performed semiempirically from equivalent reflections on basis of multi-scans (Bruker AXS APEX2) and numerical from indexed faces (15). Hydrogen atoms were refined using a riding model or rigid methyl groups. In 9 an ethyl group is disordered over two positions. RIGU restraints were applied to the anisotropic displacement parameters of the corresponding atoms. In 15 one ethanolate is partially (approx. 3\%) replaced by iodine and one dipp group is disordered over two positions. The phenyl rings were constrained to be a regular hexagon with edges $1.39 \AA$ of length (AFIX 66). All corresponding bond lengths and angles of the iso-propyl groups were restrained to be equal (SADI). RIGU restraints were applied to the anisotropic displacement parameters of all disordered atoms. Additional SIMU restraints were used for the dipp groups. The $n$-hexane molecule is disordered over a center of inversion. Its atoms were refined with common displacement parameters (EADP). The high residual electron density in the vicinity of the Bi atoms suggests remaining absorption problems. Several methods and parameters were tried but no further improvement could be achieved. Consequently, quantitative results should be carefully assessed.

CCDC-2077926 (8), -2077927 (9), -2077928 (10), -2077929 (11), -2077930 (13), and -2077931 (15) contain the supplementary crystallographic data for this paper. These data can be obtained free of charge from The Cambridge Crystallographic Data Centre via www.ccdc.cam.ac.uk/data request/cif.

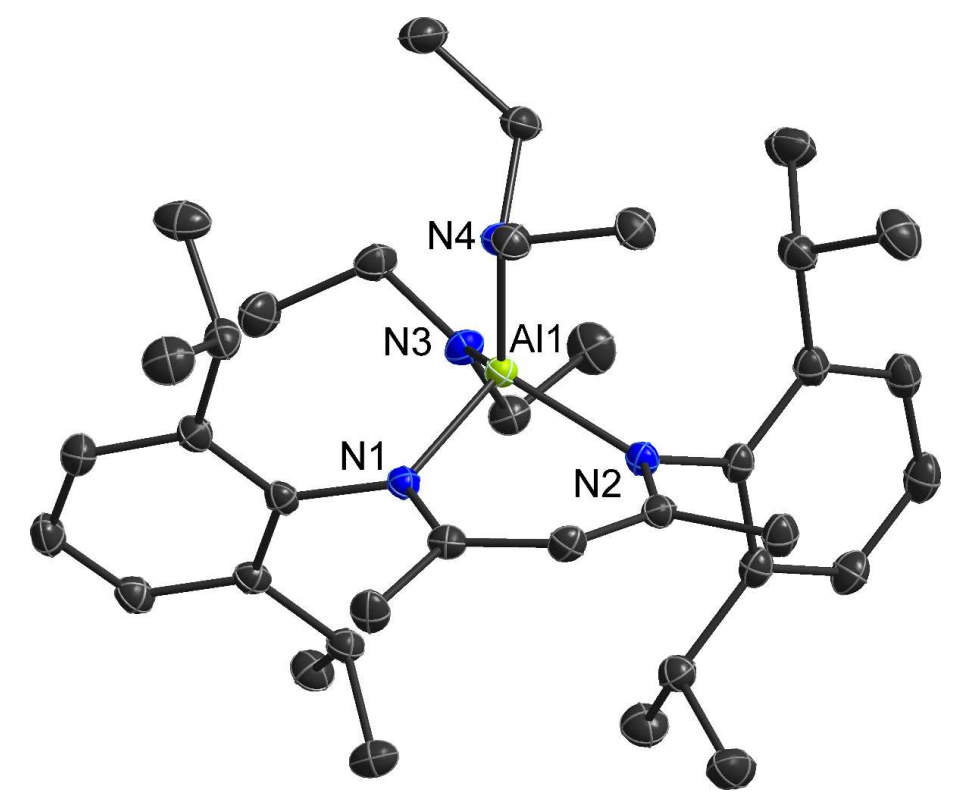

Fig. S 65. Molecular structure of $\mathrm{LAl}\left(\mathrm{NEt}_{2}\right)_{2} \mathbf{1 3}$ in the crystal. H-atoms have been omitted for clarity. Displacement ellipsoids are drawn at the $50 \%$ probability level. 
Table S3a. Crystallographic data of $\left[\mathrm{L}\left(\mathrm{Me}_{2} \mathrm{~N}\right) \mathrm{AlSb}\right]_{2}(\mathbf{8})$, $\left[\mathrm{L}\left(\mathrm{Et}_{2} \mathrm{~N}\right) \mathrm{AlBi}\right]_{2}(\mathbf{9})$, and $\left[\mathrm{L}\left(\mathrm{Me}_{2} \mathrm{~N}\right) \mathrm{GaBi}\right]_{2}(\mathbf{1 0})$.

\begin{tabular}{|c|c|c|c|}
\hline & 8 & 9 & 10 \\
\hline Empirical formula & $\mathrm{C}_{62} \mathrm{H}_{94} \mathrm{Al}_{2} \mathrm{~N}_{6} \mathrm{Sb}_{2}$ & $\mathrm{C}_{66} \mathrm{H}_{102} \mathrm{Al}_{2} \mathrm{Bi}_{2} \mathrm{~N}_{6}$ & $\mathrm{C}_{62} \mathrm{H}_{94} \mathrm{Bi}_{2} \mathrm{Ga}_{2} \mathrm{~N}_{6}$ \\
\hline $\mathrm{M}$ & 1220.89 & 1451.45 & 1480.83 \\
\hline Crystal size $[\mathrm{mm}]$ & $0.293 \times 0.171 \times 0.087$ & $0.101 \times 0.053 \times 0.045$ & $0.304 \times 0.287 \times 0.152$ \\
\hline $\mathrm{T}[\mathrm{K}]$ & $100(2)$ & $100(2)$ & $100(2)$ \\
\hline Crystal system & triclinic & monoclinic & triclinic \\
\hline Space group & $P-1$ & $P 2_{1} / n$ & $P-1$ \\
\hline $\mathrm{a}[\AA ̊]$ & $10.5975(14)$ & $11.331(4)$ & $10.6015(7)$ \\
\hline $\mathrm{b}[\AA]$ & $12.2926(16)$ & $13.980(4)$ & $12.3243(8)$ \\
\hline $\mathrm{c}[\AA]$ & $14.1227(19)$ & $20.635(6)$ & $14.1072(9)$ \\
\hline$\alpha\left[^{\circ}\right]$ & $107.189(3)$ & 90 & $106.8132(11)$ \\
\hline$\beta\left[^{\circ}\right]$ & $103.366(3)$ & $90.306(7)$ & $103.0605(12)$ \\
\hline$\gamma\left[^{\circ}\right]$ & $109.673(3)$ & 90 & $109.7870(12)$ \\
\hline $\mathrm{V}\left[\AA^{3}\right]$ & $1539.2(4)$ & $3268.9(17)$ & $1549.17(17)$ \\
\hline Z & 1 & 2 & 1 \\
\hline Dcalc $[\mathrm{g} \cdot \mathrm{cm}-3]$ & 1.317 & 1.475 & 1.587 \\
\hline$\mu\left(\mathrm{Mo} / \mathrm{CuK}_{\alpha}\right)\left[\mathrm{mm}^{-1}\right]$ & 7.544 & 5.445 & 12.242 \\
\hline Transmissions & $0.75 / 0.47$ & $0.75 / 0.61$ & $0.75 / 0.35$ \\
\hline $\mathrm{F}(000)$ & 636 & 1464 & 736 \\
\hline \multirow[t]{3}{*}{ Index ranges } & $-13 \leq h \leq 11$ & $-17 \leq h \leq 17$ & $-13 \leq h \leq 13$ \\
\hline & $-15 \leq k \leq 15$ & $-21 \leq k \leq 21$ & $-14 \leq k \leq 15$ \\
\hline & $-17 \leq l \leq 18$ & $-31 \leq l \leq 31$ & $-18 \leq l \leq 18$ \\
\hline$\theta_{\max }\left[{ }^{\circ}\right]$ & 81.153 & 33.138 & 80.795 \\
\hline Refl. collected & 148073 & 78359 & 72163 \\
\hline Independ. refl. & 6690 & 12457 & 6737 \\
\hline $\mathrm{R}_{\text {int }}$ & 0.0446 & 0.0990 & 0.0473 \\
\hline Refined parameters & 337 & 375 & 337 \\
\hline $\mathrm{R}_{1}[\mathrm{I}>2 \sigma(\mathrm{I})]$ & 0.0209 & 0.0422 & 0.0286 \\
\hline $\mathrm{wR}_{2}$ [all data] & 0.0570 & 0.0940 & 0.0745 \\
\hline GooF & 1.041 & 1.003 & 1.043 \\
\hline $\begin{array}{c}\Delta \rho_{\text {final }}(\max / \min ) \\
{\left[\mathrm{e} \cdot \AA^{-3}\right]}\end{array}$ & $0.656 /-0.914$ & $2.336 /-1.379$ & $2.104 /-1.551$ \\
\hline
\end{tabular}


Table S3b. Crystallographic data of $[\mathrm{L}(\mathrm{EtO}) \mathrm{GaBi}]_{2}(\mathbf{1 1}), \mathrm{LAl}\left(\mathrm{NEt}_{2}\right)_{2}(\mathbf{1 3})$, and $\left[\left\{\mathrm{L}(\mathrm{EtO}) \mathrm{Ga}_{2}-\mu, \eta^{1: 1}-\mathrm{Bi}_{4}\right]\right.$ (15).

\begin{tabular}{|c|c|c|c|}
\hline & 11 & 13 & 15 \\
\hline Empirical formula & $\mathrm{C}_{62} \mathrm{H}_{92} \mathrm{Bi}_{2} \mathrm{Ga}_{2} \mathrm{~N}_{4} \mathrm{O}_{2}$ & $\mathrm{C}_{37} \mathrm{H}_{61} \mathrm{AlN}_{4}$ & $\mathrm{C}_{64.96} \mathrm{H}_{98.89} \mathrm{Bi}_{4} \mathrm{Ga}_{2} \mathrm{~N}_{4} \mathrm{O}_{1.98}$ \\
\hline M & 1482.79 & 588.87 & 1945.64 \\
\hline Crystal size $[\mathrm{mm}]$ & $0.357 \times 0.288 \times 0.180$ & $0.126 \times 0.124 \times 0.082$ & $0.154 \times 0.056 \times 0.055$ \\
\hline $\mathrm{T}[\mathrm{K}]$ & $100(2)$ & $100(2)$ & $100(2)$ \\
\hline Crystal system & orthorhombic & monoclinic & monoclinic \\
\hline Space group & $P b c a$ & $P 2_{1} / c$ & $P 2_{1} / c$ \\
\hline $\mathrm{a}[\AA]$ & $16.0046(5)$ & $19.6396(7)$ & $20.211(2)$ \\
\hline $\mathrm{b}[\AA]$ & $18.5086(6)$ & $11.3304(4)$ & $17.2577(19)$ \\
\hline c $[\AA]$ & $21.2763(6)$ & $16.2478(6)$ & $20.781(2)$ \\
\hline$\alpha\left[^{\circ}\right]$ & 90 & 90 & 90 \\
\hline$\beta\left[^{\circ}\right]$ & 90 & $100.7950(15)$ & $106.208(4)$ \\
\hline$\gamma\left[^{\circ}\right]$ & 90 & 90 & 90 \\
\hline $\mathrm{V}\left[\AA^{3}\right]$ & $6302.5(3)$ & $3551.6(2)$ & $6960.3(13)$ \\
\hline Z & 4 & 4 & 4 \\
\hline Dcalc $[\mathrm{g} \cdot \mathrm{cm}-3]$ & 1.563 & 1.101 & 1.857 \\
\hline$\mu\left(\mathrm{Mo} / \mathrm{CuK}_{\alpha}\right)\left[\mathrm{mm}^{-1}\right]$ & 6.455 & 0.707 & 10.892 \\
\hline Transmissions & $0.75 / 0.48$ & $0.75 / 0.68$ & $0.17 / 0.05$ \\
\hline $\mathrm{F}(000)$ & 2944 & 1296 & 3710 \\
\hline \multirow[t]{3}{*}{ Index ranges } & $-26 \leq h \leq 26$ & $-24 \leq h \leq 21$ & $-27 \leq \mathrm{h} \leq 27$ \\
\hline & $-30 \leq k \leq 30$ & $-14 \leq k \leq 14$ & $-23 \leq \mathrm{k} \leq 23$ \\
\hline & $-35 \leq l \leq 35$ & $-20 \leq l \leq 20$ & $-27 \leq 1 \leq 27$ \\
\hline$\theta_{\max }\left[{ }^{\circ}\right]$ & 36.428 & 80.903 & 28.418 \\
\hline $\begin{array}{l}\text { Reflections } \\
\text { collected }\end{array}$ & 481040 & 120373 & 135672 \\
\hline $\begin{array}{l}\text { Independ. } \\
\text { reflections }\end{array}$ & 15367 & 7788 & 17412 \\
\hline $\mathrm{R}_{\text {int }}$ & 0.0655 & 0.0444 & 0.0626 \\
\hline Refined parameters & 336 & 393 & 811 \\
\hline $\mathrm{R}_{1}[\mathrm{I}>2 \sigma(\mathrm{I})]$ & 0.0255 & 0.0346 & 0.0448 \\
\hline $\mathrm{wR}_{2}$ [all data] & 0.0530 & 0.0930 & 0.0976 \\
\hline GooF & 1.159 & 1.030 & 1.045 \\
\hline $\begin{array}{c}\Delta \rho_{\text {final }}(\max / \min ) \\
{\left[\mathrm{e} \cdot \AA^{-3}\right]}\end{array}$ & $1.634 /-1.438$ & $0.279 /-0.282$ & $7.197 /-2.935$ \\
\hline
\end{tabular}




\section{Computational details}

The ORCA quantum chemistry package version (4.2.1) ${ }^{10-12}$ was used for the DFT calculations. The geometric parameters of the species were optimized in the gas phase employing the PBE0 density functional ${ }^{13}$ with ultrafine grid and Def2-SVP ${ }^{14}$ basis set on $\mathrm{H}, \mathrm{C}, \mathrm{N}$ and Def2-TZVP ${ }^{14}$ on Al, Ga, $\mathrm{Sb}$, utilizing atom-pairwise dispersion correction with Becke-Johnson damping scheme (D3BJ). ${ }^{15,16}$ A small effective core potential was employed on $\mathrm{Sb}^{17}$ To accelerate the calculations the RIJCOSX approximation with ultrafine grid was utilized with the def-2J auxiliary basis sets. ${ }^{18}$ Frequency calculations were carried out for all optimized structures (no imaginary frequencies were found). The electron densities and frontier molecular orbitals were calculated with Def2-TZVP ${ }^{14}$ basis set on $\mathrm{H}, \mathrm{C}$, and Def2-QZVPP ${ }^{14}$ on $\mathrm{Al}, \mathrm{Ga}, \mathrm{Sb}$. Natural bond orbital analysis was performed using the NBO 6.0 program. ${ }^{19}$

Atoms in molecules (AIM) ${ }^{19-22}$ and electron localization function (ELF) $)^{23,24}$ computations were performed with the Multiwfn program $3.7^{25}$ replacing the inner-core density by a pseudopotential. ${ }^{26}$ The high-quality rectangular grid with a mesh size of $0.06 \mathrm{Bohr}$ with basin boundary refinement was employed. VMD was developed by the Theoretical and Computational Biophysics Group in the Beckman Institute for Advanced Science and Technology at the University of Illinois at Urbana-Champaign and used to plot MO. ${ }^{27}$
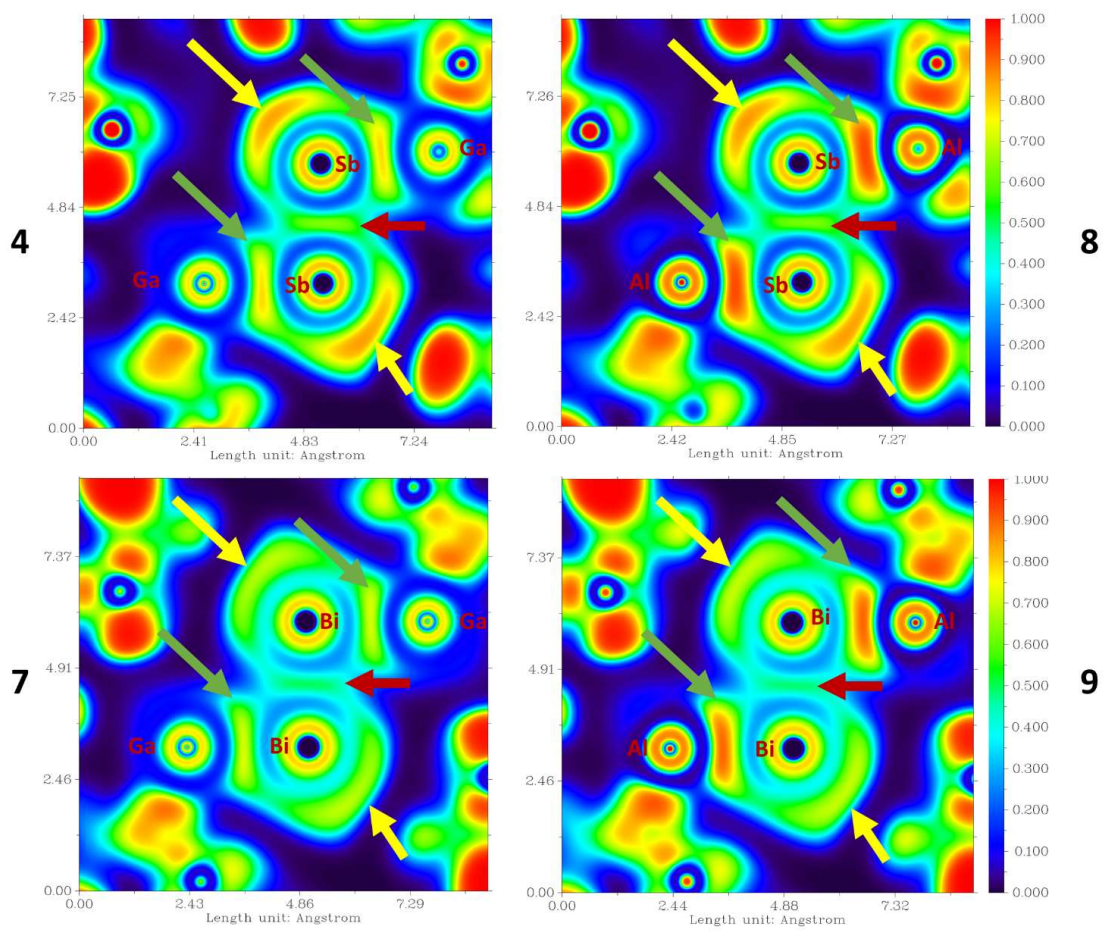

Fig. S66. ELF distribution in the $\left[\left(\mathrm{L}\left(\mathrm{Me}_{2} \mathrm{~N}\right) \mathrm{AlSb}\right]_{2} \mathbf{8},\left[\left(\mathrm{L}\left(\mathrm{Me}_{2} \mathrm{~N}\right) \mathrm{GaSb}\right]_{2} \mathbf{4}\left[\left(\mathrm{L}_{(\mathrm{Et}} \mathrm{N}\right) \mathrm{AlBi}\right]_{2} \mathbf{9}\right.\right.$ and $\left[\left(\mathrm{L}\left(\mathrm{Et}_{2} \mathrm{~N}\right) \mathrm{GaBi}\right]_{2} 7\right.$ in the $\mathrm{M}-\mathrm{E}-\mathrm{E}-\mathrm{M}$ plane, left to right. $\mathrm{V}(\mathrm{M}, \mathrm{E}), \mathrm{V}(\mathrm{E})$, and $\mathrm{V}(\mathrm{E}, \mathrm{E})$ basins are indicated by green, yellow, and red arrows, respectively. 
Table S 4. Calculated X-Y bond lengths (r, Å) (exp.), X and Y NPA (AIM) atomic charges (q, ee $\mid$ ), Wiberg bond indices (WBI), occupation numbers $(\mathrm{ON},|\mathrm{e}|)$ of the $\sigma \mathrm{XY}$ bonds according to NBO analysis, populations of $\mathrm{V}(\mathrm{X}, \mathrm{Y})$ ELF basins $(\bar{N}[\mathrm{~V}(\mathrm{X}, \mathrm{Y})]$, e $)$, and AIM parameters at the bond critical points $\left(\rho\left(\mathrm{r}_{\mathrm{b}}\right), \Delta \rho\left(\mathrm{r}_{\mathrm{b}}\right), \mid \mathrm{V}\left(\mathrm{r}_{\mathrm{b}}\right) / / \mathrm{G}\left(\mathrm{r}_{\mathrm{b}}\right), \mathrm{H}\left(\mathrm{r}_{\mathrm{b}}\right)\right.$, atomic units) for the $\mathrm{Ga}_{2} \mathrm{Sb}_{2}$ skeleton of $\left[\mathrm{L}\left(\mathrm{NMe}_{2}\right) \mathrm{GaSb}\right]_{2} 4$.

\begin{tabular}{|c|c|c|c|c|c|c|c|c|c|c|}
\hline $\mathrm{X}-\mathrm{Y}$ & $\mathrm{r}(\mathrm{X}-\mathrm{Y})$ & $\mathrm{q}(\mathrm{X})$ & $\mathrm{q}(\mathrm{Y})$ & WBI & $\mathrm{ON}^{[\mathrm{a}]}$ & $\bar{N}[V(X, Y)]^{[\mathrm{b}]}$ & $\rho\left(r_{b}\right)$ & $\Delta \rho\left(r_{b}\right)$ & $\left|\mathrm{V}\left(\mathrm{r}_{\mathrm{b}}\right)\right| / \mathrm{G}\left(\mathrm{r}_{\mathrm{b}}\right)$ & $\mathrm{H}\left(\mathrm{r}_{\mathrm{b}}\right)$ \\
\hline $\mathrm{Ga} 2-\mathrm{Sb} 1$ & $\begin{array}{c}2.60 \\
(2.62)\end{array}$ & $\begin{array}{c}1.36 \\
(1.37)\end{array}$ & $\begin{array}{c}-0.16 \\
(-0.16)\end{array}$ & 0.97 & $\begin{array}{c}1.96 \\
0.392 / 0.608\end{array}$ & $\begin{array}{c}2.259 \\
0.975 / 1.264\end{array}$ & 0.069 & -0.030 & 2.384 & -0.027 \\
\hline Ga85-Sb84 & $\begin{array}{c}2.60 \\
(2.62)\end{array}$ & $\begin{array}{c}1.38 \\
(1.34)\end{array}$ & $\begin{array}{c}-0.16 \\
(-0.16)\end{array}$ & 0.96 & $\begin{array}{c}1.96 \\
0.390 / 0.601\end{array}$ & $\begin{array}{c}2.297 \\
0.997 / 1.280\end{array}$ & 0.069 & -0.031 & 2.406 & -0.027 \\
\hline $\mathrm{Sb} 1-\mathrm{Sb} 84$ & $\begin{array}{c}2.62 \\
(2.65)\end{array}$ & & & 1.82 & $\begin{array}{c}\sigma 1.95 \\
0.502 / 0.498 \\
\pi 1.91 \\
0.501 / 0.499\end{array}$ & $\begin{array}{c}1.438 \\
0.660 / 0.764 \\
1.410 \\
0.728 / 0.671\end{array}$ & 0.076 & -0.011 & 2.009 & -0.030 \\
\hline $\begin{array}{l}\text { Sb1 lone- } \\
\text { Sb84 pair }\end{array}$ & & & & & $\begin{array}{l}1.94 \\
1.93\end{array}$ & $\begin{array}{l}2.720 / 2.691 \\
2.619 / 2.584\end{array}$ & & & & \\
\hline
\end{tabular}

[a]: Squared polarization coefficients $\mathrm{c}_{\mathrm{X}}\left(\left|\mathrm{c}_{\mathrm{X}}\right|^{2}\right)$ of the $\sigma \mathrm{XY}$ bond NBOs. [b]: Contributions of $\mathrm{X}_{1}-\mathrm{X}_{2}$ electrons into the basins $\left(\bar{N}\left[\mathrm{~V}\left(\mathrm{X}_{1}, \mathrm{X}_{2}\right) \mid \mathrm{X}_{1} / \mathrm{X}_{2}\right], \mathrm{e}\right)$ are given. 
Table S 5. Calculated X-Y bond lengths (r, Å) (exp.), X and Y NPA (AIM) atomic charges (q, ee $\mid$ ), Wiberg bond indices (WBI), occupation numbers $(\mathrm{ON},|\mathrm{e}|)$ of the $\sigma \mathrm{XY}$ bonds according to NBO analysis, populations of $\mathrm{V}(\mathrm{X}, \mathrm{Y})$ ELF basins $(\bar{N}[\mathrm{~V}(\mathrm{X}, \mathrm{Y})]$, e $)$, and AIM parameters at the bond critical points $\left(\rho\left(\mathrm{r}_{\mathrm{b}}\right), \Delta \rho\left(\mathrm{r}_{\mathrm{b}}\right),\left|\mathrm{V}\left(\mathrm{r}_{\mathrm{b}}\right)\right| / \mathrm{G}\left(\mathrm{r}_{\mathrm{b}}\right), \mathrm{H}\left(\mathrm{r}_{\mathrm{b}}\right)\right.$, atomic units) for the $\mathrm{Ga}_{2} \mathrm{Al}_{2}$ skeleton of [LAl( $\left.\left.\mathrm{Me}_{2} \mathrm{~N}\right) \mathrm{AlSb}\right]_{2} 8$.

\begin{tabular}{|c|c|c|c|c|c|c|c|c|c|c|}
\hline$X-Y$ & $r(X-Y)$ & $q(X)$ & $\mathrm{q}(\mathrm{Y})$ & WBI & $\mathrm{ON}^{[a]}$ & $\bar{N}[V(X, Y)]^{[\mathrm{b}]}$ & $\rho\left(\mathrm{r}_{\mathrm{b}}\right)$ & $\Delta \rho\left(\mathrm{r}_{\mathrm{b}}\right)$ & $\left|\mathrm{V}\left(\mathrm{r}_{\mathrm{b}}\right)\right| / \mathrm{G}\left(\mathrm{r}_{\mathrm{b}}\right)$ & $\mathrm{H}\left(\mathrm{r}_{\mathrm{b}}\right)$ \\
\hline Al1-Sb2 & $\begin{array}{c}2.64 \\
(2.67)\end{array}$ & $\begin{array}{c}1.69 \\
(2.33)\end{array}$ & $\begin{array}{c}-0.29 \\
(-0.64)\end{array}$ & 0.89 & $\begin{array}{c}1.92 \\
0.292 / 0.708\end{array}$ & $\begin{array}{c}2.188 \\
0.281 / 1.860\end{array}$ & 0.057 & -0.0124 & 1.890 & -0.025 \\
\hline A184-Sb85 & $\begin{array}{c}2.64 \\
(2.67)\end{array}$ & $\begin{array}{c}1.69 \\
(2.34)\end{array}$ & $\begin{array}{c}-0.30 \\
(-0.62)\end{array}$ & 0.89 & $\begin{array}{c}1.94 \\
0.29 / 0.709\end{array}$ & $\begin{array}{c}2.212 \\
0.287 / 1.878\end{array}$ & 0.057 & -0.0102 & 1.908 & -0.025 \\
\hline $\mathrm{Sb} 2-\mathrm{Sb} 85$ & $\begin{array}{c}2.63 \\
(2.66)\end{array}$ & & & 1.81 & $\begin{array}{c}\sigma 1.95 \\
0.499 / 0.501 \\
\pi 1.91 \\
0.500 / 0.500\end{array}$ & $\begin{array}{c}1.331 \\
0.683 / 0.637 \\
1.356 \\
0.622 / 0.720\end{array}$ & 0.075 & -0.0020 & 1.983 & -0.029 \\
\hline $\begin{array}{l}\text { Sb2 lone- } \\
\text { Sb85 pair }\end{array}$ & & & & & $\begin{array}{l}1.94 \\
1.93\end{array}$ & $\begin{array}{c}2.686 / 2.654 \\
2.593 / 2.556\end{array}$ & & & & \\
\hline
\end{tabular}

[a]: Squared polarization coefficients $\mathrm{c}_{\mathrm{X}}\left(\left|\mathrm{c}_{\mathrm{X}}\right|^{2}\right)$ of the $\sigma \mathrm{XY}$ bond NBOs. [b]: Contributions of $\mathrm{X}_{1}-\mathrm{X}_{2}$ electrons into the basins $\left(\bar{N}\left[\mathrm{~V}\left(\mathrm{X}_{1}, \mathrm{X}_{2}\right) \mid \mathrm{X}_{1} / \mathrm{X}_{2}\right]\right.$, e $)$ are given. 
Table S 6. Calculated X-Y bond lengths (r, Å) (exp.), X and Y NPA (AIM) atomic charges (q, ee $\mid$ ), Wiberg bond indices (WBI), occupation numbers $(\mathrm{ON},|\mathrm{e}|)$ of the $\sigma \mathrm{XY}$ bonds according to NBO analysis, populations of $\mathrm{V}(\mathrm{X}, \mathrm{Y})$ ELF basins $(\bar{N}[\mathrm{~V}(\mathrm{X}, \mathrm{Y})]$, e), and AIM parameters at the bond critical points $\left(\rho\left(\mathrm{r}_{\mathrm{b}}\right), \Delta \rho\left(\mathrm{r}_{\mathrm{b}}\right),\left|\mathrm{V}\left(\mathrm{r}_{\mathrm{b}}\right)\right| / \mathrm{G}\left(\mathrm{r}_{\mathrm{b}}\right), \mathrm{H}\left(\mathrm{r}_{\mathrm{b}}\right)\right.$, atomic units) for the $\mathrm{Ga}_{2} \mathrm{Bi}_{2}$ skeleton of $\left[\mathrm{L}\left(\mathrm{Et}_{2} \mathrm{~N}\right) \mathrm{GaBi}\right]_{2} 7$.

\begin{tabular}{|c|c|c|c|c|c|c|c|c|c|c|}
\hline $\mathrm{X}-\mathrm{Y}$ & $r(X-Y)$ & $q(X)$ & $\mathrm{q}(\mathrm{Y})$ & WBI & $\mathrm{ON}^{[\mathrm{a}]}$ & $\bar{N}[V(X, Y)]^{[\mathrm{b}]}$ & $\rho\left(r_{b}\right)$ & $\Delta \rho\left(r_{b}\right)$ & $\left|\mathrm{V}\left(\mathrm{r}_{\mathrm{b}}\right)\right| / \mathrm{G}\left(\mathrm{r}_{\mathrm{b}}\right)$ & $\mathrm{H}\left(\mathrm{r}_{\mathrm{b}}\right)$ \\
\hline Ga2-Bi1 & $\begin{array}{c}2.67 \\
(2.71)\end{array}$ & $\begin{array}{c}1.33 \\
(1.33)\end{array}$ & $\begin{array}{c}-0.10 \\
(-0.11)\end{array}$ & 0.98 & $\begin{array}{c}1.96 \\
0.411 / 0.589\end{array}$ & $\begin{array}{c}2.216 \\
1.031 / 1.161\end{array}$ & 0.065 & -0.0154 & 2.199 & -0.023 \\
\hline Ga91-Bi90 & $\begin{array}{c}2.67 \\
(2.71)\end{array}$ & $\begin{array}{c}1.33 \\
(1.36)\end{array}$ & $\begin{array}{c}-0.10 \\
(-0.20)\end{array}$ & 0.98 & $\begin{array}{c}1.96 \\
0.412 / 0.588\end{array}$ & $\begin{array}{c}2.216 \\
1.031 / 1.161\end{array}$ & 0.065 & -0.0154 & 2.199 & -0.023 \\
\hline Bi1-Bi90 & $\begin{array}{c}2.77 \\
(2.81)\end{array}$ & & & 1.81 & $\begin{array}{c}\sigma 1.94 \\
0.499 / 0.501 \\
\pi 1.90 \\
0.500 / 0.500\end{array}$ & $\begin{array}{c}1.323 \\
0.693 / 0.617 \\
1.324 \\
0.617 / 0.693\end{array}$ & 0.066 & -0.0429 & 1.655 & -0.020 \\
\hline $\begin{array}{l}\text { Bi1 lone- } \\
\text { Bi90 pair }\end{array}$ & & & & & $\begin{array}{l}1.95 \\
1.95\end{array}$ & $\begin{array}{c}2.860 / 2.830 \\
2.860 / 2.831\end{array}$ & & & & \\
\hline
\end{tabular}

[a]: Squared polarization coefficients $\mathrm{c}_{\mathrm{X}}\left(\left|\mathrm{c}_{\mathrm{X}}\right|^{2}\right)$ of the $\sigma \mathrm{XY}$ bond NBOs. [b]: Contributions of $\mathrm{X}_{1}-\mathrm{X}_{2}$ electrons into the basins $\left(\underline{N}\left[\mathrm{~V}\left(\mathrm{X}_{1}, \mathrm{X}_{2}\right) \mid \mathrm{X}_{1} / \mathrm{X}_{2}\right]\right.$, e $)$ are given. 
Table S 7. Calculated X-Y bond lengths (r, Å) (exp.), X and Y NPA (AIM) atomic charges (q, ee $\mid$ ), Wiberg bond indices (WBI), occupation numbers $(\mathrm{ON},|\mathrm{e}|)$ of the $\sigma \mathrm{XY}$ bonds according to NBO analysis, populations of $\mathrm{V}(\mathrm{X}, \mathrm{Y})$ ELF basins $(\bar{N}[\mathrm{~V}(\mathrm{X}, \mathrm{Y})]$, e), and AIM parameters at the bond critical points $\left(\rho\left(\mathrm{r}_{\mathrm{b}}\right), \Delta \rho\left(\mathrm{r}_{\mathrm{b}}\right),\left|\mathrm{V}\left(\mathrm{r}_{\mathrm{b}}\right)\right| / \mathrm{G}\left(\mathrm{r}_{\mathrm{b}}\right), \mathrm{H}\left(\mathrm{r}_{\mathrm{b}}\right)\right.$, atomic units) for the $\mathrm{Ga}_{2} \mathrm{Bi}_{2}$ skeleton of $\left[\mathrm{L}\left(\mathrm{Et}_{2} \mathrm{~N}\right) \mathrm{AlBi}\right]_{2} 9$.

\begin{tabular}{|c|c|c|c|c|c|c|c|c|c|c|}
\hline $\mathrm{X}-\mathrm{Y}$ & $r(X-Y)$ & $q(X)$ & $\mathrm{q}(\mathrm{Y})$ & WBI & $\mathrm{ON}^{[\mathrm{a}]}$ & $\bar{N}[V(X, Y)]^{[\mathrm{b}]}$ & $\rho\left(r_{b}\right)$ & $\Delta \rho\left(\mathrm{r}_{\mathrm{b}}\right)$ & $\left|\mathrm{V}\left(\mathrm{r}_{\mathrm{b}}\right)\right| / \mathrm{G}\left(\mathrm{r}_{\mathrm{b}}\right)$ & $\mathrm{H}\left(\mathrm{r}_{\mathrm{b}}\right)$ \\
\hline A12-Bi1 & $\begin{array}{c}2.71 \\
(2.73)\end{array}$ & $\begin{array}{c}1.64 \\
(2.31)\end{array}$ & $\begin{array}{c}-0.24 \\
(-0.58)\end{array}$ & 0.92 & $\begin{array}{c}1.96 \\
0.325 / 0.675\end{array}$ & $\begin{array}{c}2.148 \\
0.322 / 1.771\end{array}$ & 0.054 & -0.0073 & 2.082 & -0.024 \\
\hline A191-Bi90 & $\begin{array}{c}2.71 \\
(2.73)\end{array}$ & $\begin{array}{c}1.64 \\
(2.31)\end{array}$ & $\begin{array}{c}-0.24 \\
(-0.58)\end{array}$ & 0.92 & $\begin{array}{c}1.96 \\
0.324 / 0.676\end{array}$ & $\begin{array}{c}2.147 \\
0.332 / 1.771\end{array}$ & 0.054 & -0.0073 & 2.081 & -0.024 \\
\hline Bi1-Bi90 & $\begin{array}{l}2.76 \\
(2.81)\end{array}$ & & & 1.80 & $\begin{array}{c}\sigma 1.94 \\
0.500 / 0.500 \\
\pi 1.89 \\
0.500 / 0.500\end{array}$ & $\begin{array}{c}1.229 \\
0.575 / 0.640 \\
1.232 \\
0.643 / 0.577\end{array}$ & 0.065 & -0.045 & 1.636 & -0.020 \\
\hline $\begin{array}{l}\text { Bi1 lone- } \\
\text { Bi90 pair }\end{array}$ & & & & & $\begin{array}{l}1.94 \\
1.94\end{array}$ & $\begin{array}{c}2.841 / 2.808 \\
2.840 / 2.808\end{array}$ & & & & \\
\hline
\end{tabular}

[a]: Squared polarization coefficients $\mathrm{c}_{\mathrm{X}}\left(\left|\mathrm{c}_{\mathrm{X}}\right|^{2}\right)$ of the $\sigma \mathrm{XY}$ bond NBOs. [b]: Contributions of $\mathrm{X}_{1}-\mathrm{X}_{2}$ electrons into the basins $\left(\underline{N}\left[\mathrm{~V}\left(\mathrm{X}_{1}, \mathrm{X}_{2}\right) \mid \mathrm{X}_{1} / \mathrm{X}_{2}\right]\right.$, e $)$ are given. 


\section{References}

(1) A. L. Spek. PLATON:A Multipurpose Crystallographic Tool; Utrecht University. https://www.platonsoft.nl/platon/p1000000.html (accessed 2021-02-12).

(2) Azor, L.; Bailly, C.; Brelot, L.; Henry, M.; Mobian, P.; Dagorne, S. Stereoselective synthesis of biphenolate/binaphtolate titanate and zirconate alkoxide species: structural characterization and use in the controlled ROP of lactide. Inorg. Chem. 2012, 51, 10876-10883

(3) Bondi, A. van der Waals Volumes and Radii. J. Phys. Chem. 1964, 68, 441-451

(4) Gavezzotti, A. The calculation of molecular volumes and the use of volume analysis in the investigation of structured media and of solid-state organic reactivity. J. Am. Chem. Soc. 1983, 105, 5220-5225.

(5) Ghosh, S.; Spannenberg, A.; Mejía, E. Cubane-Type Polynuclear Zinc Complexes Containing Tridentate Schiff Base Ligands: Synthesis, Characterization, and Ring-Opening Polymerization Studies of rac -Lactide and $\varepsilon$-Caprolactone. Helv. Chim. Acta 2017, 100, 1-17.

(6) Evans, D. F. 400. The determination of the paramagnetic susceptibility of substances in solution by nuclear magnetic resonance. J. Chem. Soc. 1959, 2003-2005.

(7) G. M. Sheldrick, Acta Crystallogr. 1990, A46, 467-472.

(8) G. M. Sheldrick, SHELXL-2014, Program for the Refinement of Crystal Structures University of Göttingen, Germany, 2014. See also: Sheldrick, G. M. Acta Crystallogr. 2008, A64, 112-122.

(9) C. B. Hübschle, G. M. Sheldrick, B. Dittrich, J. Appl. Cryst. 2011, 44, 1281-1284.

(10) Neese, F. Software update: the ORCA program system, version 4.0. WIREs Comput Mol Sci 2018, 8, $1-6$.

(11) Neese, F.; Wennmohs, F.; Becker, U.; Riplinger, C. The ORCA quantum chemistry program package. J. Chem. Phys. 2020, 152, 224108.

(12) Neese, F. The ORCA program system. WIREs Comput Mol Sci 2012, 2, 73-78.

(13) Adamo, C.; Barone, V. Toward reliable density functional methods without adjustable parameters: The PBE0 model. J. Chem. Phys. 1999, 110, 6158-6170.

(14) Weigend, F.; Ahlrichs, R. Balanced basis sets of split valence, triple zeta valence and quadruple zeta valence quality for $\mathrm{H}$ to $\mathrm{Rn}$ : Design and assessment of accuracy. PCCP 2005, 7, 3297-3305.

(15) Grimme, S.; Ehrlich, S.; Goerigk, L. Effect of the damping function in dispersion corrected density functional theory. J. Comput. Chem. 2011, 32, 1456-1465.

(16) Grimme, S.; Antony, J.; Ehrlich, S.; Krieg, H. A consistent and accurate ab initio parametrization of density functional dispersion correction (DFT-D) for the 94 elements H-Pu. J. Chem. Phys. 2010, 132, 154104.

(17) Metz, B.; Stoll, H.; Dolg, M. Small-core multiconfiguration-Dirac-Hartree-Fock-adjusted pseudopotentials for post- $\mathrm{d}$ main group elements: Application to PbH and PbO. J. Chem. Phys.2000, 113, 2563-2569.

(18) Weigend, F. Accurate Coulomb-fitting basis sets for H to Rn. PCCP 2006, 8, 1057-1065.

(19) E. D. Glendening, J. K. Badenhoop, A. E. Reed, J. E. Carpenter, J. A. Bohmann, C. M. Morales, C. R. Landis, and F. Weinhold. NBO 6.0.; (Theoretical Chemistry Institute, University of Wisconsin, Madison, WI, 2013). https://nbo6.chem.wisc.edu/

(20) Kalaiarasi, C.; Pavan, M. S.; Kumaradhas, P. Topological characterization of electron density, electrostatic potential and intermolecular interactions of 2-nitroimidazole: an experimental and theoretical study. Acta. Crystallogr. B 2016, 72, 775-786.

(21) Kumar, P. Shayam, V; Raghavendra, V.; Subramanian, V. Bader's Theory of Atoms in Molecules (AIM) and its Applications to Chemical Bonding. J. Chem. Sci. 2016, 128, 1527-1536. 
(22) Bader, R. F. W. A quantum theory of molecular structure and its applications. Chem. Rev. 1991, 91, 893-928.

(23) Silvi, B.; Savin, A. Classification of chemical bonds based on topological analysis of electron localization functions. Nature 1994, 371, 683-686.

(24) Becke, A. D.; Edgecombe, K. E. A simple measure of electron localization in atomic and molecular systems. J. Chem. Phys. 1990, 92, 5397-5403.

(25) Lu, T.; Chen, F. Multiwfn: a multifunctional wavefunction analyzer. J. Comput. Chem. 2012, 33, 580592.

(26) Zou, W.; Cai, Z.; Wang, J.; Xin, K. An open library of relativistic core electron density function for the QTAIM analysis with pseudopotentials. J. Comput. Chem. 2018, 39, 1697-1706.

(27) Humphrey, W.; Dalke, A.; Schulten, K. VMD: Visual molecular dynamics. Journal of Molecular Graphics 1996, 14, 33-38. http://www.ks.uiuc.edu/Research/vmd/ 